\title{
Dual Banach algebras: representations and injectivity
}

\author{
by \\ Matthew Daws (Oxford)
}

\begin{abstract}
We study representations of Banach algebras on reflexive Banach spaces. Algebras which admit such representations which are bounded below seem to be a good generalisation of Arens regular Banach algebras; this class includes dual Banach algebras as defined by Runde, but also all group algebras, and all discrete (weakly cancellative) semigroup algebras. Such algebras also behave in a similar way to $\mathrm{C}^{*}$ - and $\mathrm{W}^{*}$-algebras; we show that interpolation space techniques can be used in place of GNS type arguments. We define a notion of injectivity for dual Banach algebras, and show that this is equivalent to Connes-amenability. We conclude by looking at the problem of defining a well-behaved tensor product for dual Banach algebras.
\end{abstract}

1. Introduction. It has been known for some time (see [20] and [37]) that a Banach algebra $\mathcal{A}$ which admits a faithful representation on a reflexive Banach space has an intrinsic characterisation, namely that the weakly almost periodic functionals, written $\operatorname{WAP}\left(\mathcal{A}^{\prime}\right)$ (see below for the definition), separate the points of $\mathcal{A}$. Similarly, if we wish to find an isometric representation of this kind, we need only ask that $\operatorname{WAP}\left(\mathcal{A}^{\prime}\right)$ form a norming set for $\mathcal{A}$. We shall call $\mathcal{A}$ a $W A P$-algebra when $\mathcal{A}$ admits an isomorphic representation on a reflexive Banach space. Such algebras seem not to have been studied abstractly before, but they seem to be a good generalisation of Arens regular Banach algebras, and to form a good framework for studying dual Banach algebras.

We follow the notation of [8], writing $\langle\cdot, \cdot\rangle$ for the dual pairing between a Banach space $E$ and its dual, $E^{\prime}$. We write $\kappa_{E}: E \rightarrow E^{\prime \prime}$ for the canonical map given by $\left\langle\kappa_{E}(x), \mu\right\rangle=\langle\mu, x\rangle$ for $x \in E$ and $\mu \in E^{\prime}$. When $E$ is reflexive, we tend to identify $E$ with $E^{\prime \prime}$. We write $\mathcal{B}(E, F)$ for the space of all bounded linear operators between Banach spaces $E$ and $F$, and we denote by $\mathcal{F}(E, F)$, $\mathcal{A}(E, F), \mathcal{K}(E, F)$ and $\mathcal{W}(E, F)$ the subspaces of, respectively, finite-rank,

2000 Mathematics Subject Classification: Primary 47L10; Secondary 46B70, 46H05, 46H15, 46H99, 46M05, 43A10, 43A20, 46A25, 46A32, 46A35, 46L10, 46L06, 46M10.

Key words and phrases: dual Banach algebra, von Neumann algebra, Connesamenability, group algebra, unique predual. 
approximable, compact and weakly compact operators (so $\mathcal{A}(E, F)$ is the operator-norm closure of $\mathcal{F}(E, F)$ in $\mathcal{B}(E, F)$ ). We write $\mathcal{B}(E)$ for $\mathcal{B}(E, E)$, and so forth.

A dual Banach algebra is a Banach algebra $\mathcal{A}$ such that $\mathcal{A}=E^{\prime}$, as a Banach space, for some Banach space $E$, and such that the multiplication on $\mathcal{A}$ is separately weak*-continuous. Recall that a $\mathrm{W}^{*}$-algebra is a $\mathrm{C}^{*}$-algebra which is a dual Banach algebra. However, it is known that the multiplication (and the involution) are automatically weak ${ }^{*}$-continuous in this case. We use [32] as general references for $\mathrm{C}^{*}$ - and $\mathrm{W}^{*}$-algebras. Dual Banach algebras were introduced in [30], but had been studied previously under different names.

For a Banach algebra $\mathcal{A}$, we turn $\mathcal{A}^{\prime}$ into an $\mathcal{A}$-bimodule in the obvious way, by setting

$$
\langle a \cdot \mu, b\rangle=\langle\mu, b a\rangle, \quad\langle\mu \cdot a, b\rangle=\langle\mu, a b\rangle \quad\left(a, b \in \mathcal{A}, \mu \in \mathcal{A}^{\prime}\right) .
$$

We may then check, for a dual Banach algebra $\mathcal{A}=E^{\prime}$, that $\kappa_{E}(E)$ is a submodule of $\mathcal{A}^{\prime}=E^{\prime \prime}$. We call $E$ the predual of $\mathcal{A}$, and write $(\mathcal{A}, E)$ if we wish to stress which predual we are using, and often write $\mathcal{A}_{*}$ for $E$. In this special case, we shall often suppress the map $\kappa_{\mathcal{A}_{*}}$, and speak of $\mathcal{A}_{*}$ as being a subspace of $\mathcal{A}^{\prime}$ (see Definition 2.6 for a justification of this). We later study when such preduals are unique, both in the isometric sense (as is well-known for $\mathrm{W}^{*}$-algebras) and the isomorphic sense, which seems more natural for Banach algebras.

When $E$ is a reflexive Banach space, the projective tensor product of $E$ with its dual $E^{\prime}$, denoted by $E^{\prime} \widehat{\otimes} E$, is the canonical predual for $\mathcal{B}(E)$ (see [30]). This induces a weak*-topology on $\mathcal{B}(E)$. Recall that the norm on $E^{\prime} \widehat{\otimes} E$ is $\pi(\cdot)$, defined by

$$
\pi(\tau)=\inf \left\{\sum_{k=1}^{n}\left\|x_{k}\right\|\left\|y_{k}\right\|: \tau=\sum_{k=1}^{n} x_{k} \otimes y_{k}\right\} \quad\left(\tau \in E^{\prime} \otimes E\right) .
$$

We write $E^{\prime} \widehat{\otimes} E$ and not $E \widehat{\otimes} E^{\prime}$ as the former makes more sense when $E$ is not necessarily reflexive; the two spaces are isometrically isomorphic. Here and elsewhere, we refer the reader to [31], [13] or [14, Chapter VIII] for further details on tensor products of Banach spaces.

Let $\mathcal{A}$ be a Banach algebra, and for $\mu \in \mathcal{A}^{\prime}$, define $L_{\mu}, R_{\mu} \in \mathcal{B}\left(\mathcal{A}, \mathcal{A}^{\prime}\right)$ by

$$
L_{\mu}(a)=\mu \cdot a, \quad R_{\mu}(a)=a \cdot \mu \quad(a \in \mathcal{A}) .
$$

Then $\mu \in \operatorname{WAP}\left(\mathcal{A}^{\prime}\right)$ if and only if $L_{\mu} \in \mathcal{W}\left(\mathcal{A}, \mathcal{A}^{\prime}\right)$ (which is equivalent to $R_{\mu} \in \mathcal{W}\left(\mathcal{A}, \mathcal{A}^{\prime}\right)$ ). This notation differs from that sometimes used, but follows $[28$, Section 4], for example. It may be easily checked (as we do below) that for a dual Banach algebra $\left(\mathcal{A}, \mathcal{A}_{*}\right)$, we have $\mathcal{A}_{*} \subseteq \operatorname{WAP}\left(\mathcal{A}^{\prime}\right)$. It hence immediately follows from [37] that there exists a reflexive Banach space $E$ and an 
isometric representation $\pi: \mathcal{A} \rightarrow \mathcal{B}(E)$ (here representation simply means a homomorphism to a Banach algebra of the form $\mathcal{B}(E))$. However, it is not immediately apparent if such a representation need be weak*-continuous. As usual, we may regard representations and left modules as interchangeable, so this question is equivalent to $E$ being normal in the sense of [28]. We show below that we can indeed choose $E$ to be normal (actually, our argument is very similar to that used by Kaijser and Young, but the required machinery, interpolation spaces, shall be needed later anyway). This shows that dual Banach algebras can be thought of as "abstract" weak*-closed subalgebras of $\mathcal{B}(E)$ for reflexive Banach spaces $E$. This exactly mirrors the fact that $\mathrm{W}^{*}$-algebras are abstract von Neumann algebras, that is, weak ${ }^{*}$-closed (which in this context agrees with weak operator topology closed) self-adjoint subalgebras of $\mathcal{B}(H)$ for a Hilbert space $H$.

A derivation from a Banach algebra $\mathcal{A}$ to an $\mathcal{A}$-bimodule $E$ is a bounded linear map $d$ such that $d(a b)=a \cdot d(b)+d(a) \cdot b$. Fix $x \in E$, and define $d$ by $d(a)=a \cdot x-x \cdot a$. Then $d$ is a derivation, called an inner derivation. We say that $\mathcal{A}$ is amenable if every derivation from $\mathcal{A}$ to a dual $\mathcal{A}$ bimodule $E^{\prime}$ is inner. We refer the reader to [29] for details on amenability. Similarly, Runde defines a dual Banach algebra $\left(\mathcal{A}, \mathcal{A}_{*}\right)$ to be Connesamenable if every weak*-continuous derivation to a normal, dual $\mathcal{A}$-bimodule is inner.

For a $\mathrm{W}^{*}$-algebra $\mathcal{A}$, it is this notion of amenability which seems most natural. One of the major achievements of $\mathrm{C}^{*}$-algebra theory has been to give equivalent natural conditions for a $\mathrm{W}^{*}$-algebra to be Connes-amenable (see [29, Chapter 6]). One of these is the notion of injectivity. We define a similar (though weaker) notion for dual Banach algebras, and show that it is equivalent to Connes-amenability.

We finish the paper with a study of tensor products of dual Banach algebras. This last section is slightly more speculative, but it is the author's opinion that fully understanding tensor products seems central to understanding notions of amenability: certainly the rather well-behaved tensor products of $\mathrm{C}^{*}$-algebras play a central role in the theory of amenability for such algebras (for example, the fact that amenability is equivalent to nuclearity).

2. Basic properties of WAP and dual Banach algebras. In this section, we shall study the basic properties of dual Banach algebras, and define WAP-algebras.

Following, for example, [8], in dealing with Banach algebras, we assume, by means of standard renormings, that the product is contractive (and not merely bounded) and that a unit always has norm one. This philosophy is compatible with dual Banach algebras: 
Proposition 2.1. Let $E$ be a Banach space such that $\mathcal{A}=E^{\prime}$ admits a bounded algebra product. Then there is an equivalent norm on $E$ such that $\mathcal{A}$ becomes a Banach algebra. If $\mathcal{A}$ has a unit $e_{\mathcal{A}}$, we may choose this norm such that $\left\|e_{\mathcal{A}}\right\|=1$.

Proof. Suppose that $1<M=\sup \{\|a b\|: a, b \in \mathcal{A},\|a\|=\|b\|=1\}$, for if $M \leq 1$, we have nothing to do. Then define $\|\mu\|_{0}=M^{-1}\|\mu\|$ for $\mu \in E$. For $a \in \mathcal{A}$, we then have $\|a\|_{0}=\sup \{|\langle a, \mu\rangle|:\|\mu\| \leq M\}=M\|a\|$, so that $\|a b\|_{0}=M\|a b\| \leq M^{2}\|a\|\|b\|=\|a\|_{0}\|b\|_{0}$.

Now suppose that $\mathcal{A}$ has a unit $e_{\mathcal{A}}$. Let

$$
X=\operatorname{convex}\left\{a \cdot \mu:\|a\|_{0}=\|\mu\|_{0} \leq 1\right\} \subseteq E,
$$

so that for $\mu \in E$, we have $\mu=e_{\mathcal{A}} \cdot \mu \in\left\|e_{\mathcal{A}}\right\|_{0}\|\mu\|_{0} X$. Thus we can define $\|\cdot\|_{1}$ on $E$ by

$$
\|\mu\|_{1}=\inf \{t>0: \mu \in t X\} \quad(\mu \in E),
$$

and find that $\|\mu\|_{1} \leq\left\|e_{\mathcal{A}}\right\|_{0}\|\mu\|_{0}$. Conversely, we have $X \subseteq\left\{\mu \in E:\|\mu\|_{0} \leq 1\right\}$, so if $\|\mu\|_{1}=1$, then for each $\varepsilon>0$, we see that $\mu \in(1+\varepsilon) X \subseteq\{(1+\varepsilon) \lambda$ : $\left.\lambda \in E,\|\lambda\|_{0} \leq 1\right\}$, and so $\|\mu\|_{0} \leq 1$. Thus $\|\cdot\|_{1}$ is equivalent to $\|\cdot\|_{0}$ and hence also equivalent to $\|\cdot\|$.

Then, for $a \in \mathcal{A}$, we have

$$
\begin{aligned}
\|a\|_{1} & =\sup \{|\langle a, \mu\rangle|: \mu \in X\}=\sup \left\{\left|\sum_{j=1}^{n}\left\langle a, b_{j} \cdot \mu_{j}\right\rangle\right|: \sum_{j=1}^{n}\left\|b_{j}\right\|_{0}\left\|\mu_{j}\right\|_{0} \leq 1\right\} \\
& =\sup \left\{|\langle a, b \cdot \mu\rangle|:\|b\|_{0}=\|\mu\|_{0} \leq 1\right\}=\sup \left\{\|a b\|_{0}:\|b\|_{0}=1\right\} .
\end{aligned}
$$

Note that $\|a b\|_{0} \leq\|a\|_{1}\|b\|_{0}$ for $a, b \in \mathcal{A}$. Hence

$$
\|a b\|_{1}=\sup \left\{\|a b c\|_{0}:\|c\|_{0}=1\right\} \leq\|a\|_{1} \sup \left\{\|b c\|_{0}:\|c\|_{0}=1\right\}=\|a\|_{1}\|b\|_{1},
$$

and clearly $\left\|e_{\mathcal{A}}\right\|_{1}=1$, as required.

Let $\left(\mathcal{A}, \mathcal{A}_{*}\right)$ be a dual Banach algebra, and let $\mathcal{A}_{*}^{b}$ be the Banach space $\mathcal{A}_{*} \oplus \mathbb{C}$ with norm

$$
\|(\mu, \alpha)\|=\max (\|\mu\|,|\alpha|) \quad\left(\mu \in \mathcal{A}_{*}, \alpha \in \mathbb{C}\right) .
$$

Then $\left(\mathcal{A}_{*}^{b}\right)^{\prime}=\mathcal{A} \oplus \mathbb{C}=\mathcal{A}^{b}$ with norm

$$
\|(a, \beta)\|=\|a\|+|\beta| \quad(a \in \mathcal{A}, \beta \in \mathbb{C}) .
$$

We turn $\mathcal{A}^{b}$ into a Banach algebra by setting $(a, \alpha)(b, \beta)=(a b+\beta a+\alpha b, \alpha \beta)$. It is a simple verification that then $\left(\mathcal{A}^{b}, \mathcal{A}_{*}^{b}\right)$ is a dual Banach algebra.

We set $\left(\mathcal{A}, \mathcal{A}_{*}\right)^{\sharp}$ to be $\left(\mathcal{A}, \mathcal{A}_{*}\right)$ when $\mathcal{A}$ is unital, and to be $\left(\mathcal{A}^{b}, \mathcal{A}_{*}^{b}\right)$ otherwise. This gives us a (rather crude, it turns out) way to unitise a dual Banach algebra.

Lemma 2.2. Let $E$ be a Banach space such that $\mathcal{A}=E^{\prime}$ is a Banach algebra. Then $(\mathcal{A}, E)$ is a dual Banach algebra if and only if $\kappa_{E}(E) \subseteq \mathcal{A}^{\prime}$ is a sub-A-bimodule. 
Proof. This is a routine calculation showing that the product is separately weak*-continuous if and only if $E$ is an $\mathcal{A}$-bimodule.

We shall now recall the Arens products which shall allow us to prove some simple facts about dual Banach algebras (much in the spirit of [26]). Most of the following results are folklore (compare, for example, with [22, Section 1]) but do not appear to have formally been collected together before.

For a Banach algebra $\mathcal{A}$, we turn $\mathcal{A}^{\prime}$ into a Banach $\mathcal{A}$-bimodule in the standard way (and hence $\mathcal{A}^{\prime \prime}$ as well). We then define bilinear maps $\mathcal{A}^{\prime \prime} \times \mathcal{A}^{\prime}$ $\rightarrow \mathcal{A}^{\prime}$ and $\mathcal{A}^{\prime} \times \mathcal{A}^{\prime \prime} \rightarrow \mathcal{A}^{\prime}$ by

$$
\langle\Phi \cdot \mu, a\rangle=\langle\Phi, \mu \cdot a\rangle, \quad\langle\mu \cdot \Phi, a\rangle=\langle\Phi, a \cdot \mu\rangle \quad\left(a \in \mathcal{A}, \mu \in \mathcal{A}^{\prime}, \Phi \in \mathcal{A}^{\prime \prime}\right) .
$$

We then define two bilinear maps $\square, \diamond: \mathcal{A}^{\prime \prime} \times \mathcal{A}^{\prime \prime} \rightarrow \mathcal{A}^{\prime \prime}$ by

$$
\langle\Phi \square \Psi, \mu\rangle=\langle\Phi, \Psi \cdot \mu\rangle, \quad\langle\Phi \diamond \Psi, \mu\rangle=\langle\Psi, \mu \cdot \Phi\rangle \quad\left(\mu \in \mathcal{A}^{\prime}, \Phi, \Psi \in \mathcal{A}^{\prime \prime}\right) .
$$

We can then calculate

$$
(\mu \cdot \Phi) \cdot \Psi=\mu \cdot(\Phi \diamond \Psi), \quad \Phi \cdot(\Psi \cdot \mu)=(\Phi \square \Psi) \cdot \mu \quad\left(\mu \in \mathcal{A}^{\prime}, \Phi, \Psi \in \mathcal{A}^{\prime \prime}\right),
$$

from which it follows that $\square$ and $\diamond$ are Banach algebra products, called the first and second Arens products respectively (see [25, Section 1.4] or [8, Theorem 2.6.15] for further details). Furthermore, $\kappa_{\mathcal{A}}(a) \square \Phi=a \cdot \Phi=$ $\kappa_{\mathcal{A}}(a) \diamond \Phi$ for $a \in \mathcal{A}, \Phi \in \mathcal{A}^{\prime \prime}$, and similarly $\Phi \square \kappa_{\mathcal{A}}(a)=\Phi \cdot a=\Phi \diamond \kappa_{\mathcal{A}}(a)$. When $\square=\diamond$, we say that $\mathcal{A}$ is Arens regular, and in this case, we may check that $\left(\mathcal{A}^{\prime \prime}, \mathcal{A}^{\prime}\right)$ becomes a dual Banach algebra.

LEMMA 2.3. Let $\mathcal{A}$ be a Banach algebra, and let $\mu \in \mathcal{A}^{\prime}$. Then $\mu \in$ $\operatorname{WAP}\left(\mathcal{A}^{\prime}\right)$ if and only if $R_{\mu} \in \mathcal{W}\left(\mathcal{A}, \mathcal{A}^{\prime}\right)$, which happens if and only if $\langle\Phi \square \Psi, \mu\rangle=\langle\Phi \diamond \Psi, \mu\rangle$ for $\Phi, \Psi \in \mathcal{A}^{\prime \prime}$.

Proof. This is a simple calculation: see [9, Proposition 3.11]. A key tool is Gantmacher's theorem, which states that $T \in \mathcal{W}(E, F)$ if and only if $T^{\prime \prime}\left(E^{\prime \prime}\right) \subseteq \kappa_{F}(F)$.

We also define the two topological centres (see [23] or [9]) by

$$
\begin{aligned}
& \mathfrak{Z}_{\mathrm{t}}^{(1)}\left(\mathcal{A}^{\prime \prime}\right)=\left\{\Phi \in \mathcal{A}^{\prime \prime}: \Phi \square \Psi=\Phi \diamond \Psi\left(\Psi \in \mathcal{A}^{\prime \prime}\right)\right\}, \\
& \mathfrak{Z}_{\mathrm{t}}^{(2)}\left(\mathcal{A}^{\prime \prime}\right)=\left\{\Phi \in \mathcal{A}^{\prime \prime}: \Psi \square \Phi=\Psi \diamond \Phi\left(\Psi \in \mathcal{A}^{\prime \prime}\right)\right\} .
\end{aligned}
$$

Then the Arens products agree on either of the topological centres, each topological centre is an algebra, and $\mathfrak{Z}_{\mathrm{t}}^{(1)}\left(\mathcal{A}^{\prime \prime}\right) \cap \mathfrak{Z}_{\mathrm{t}}^{(2)}\left(\mathcal{A}^{\prime \prime}\right) \supseteq \kappa_{\mathcal{A}}(\mathcal{A})$ is an ideal in $\mathcal{A}^{\prime \prime}$ with respect to either Arens product.

For a Banach space $E$, a subspace $F$ of $E$, and a subspace $G$ of $E^{\prime}$, we define

$$
F^{\perp}=\left\{\mu \in E^{\prime}:\langle\mu, x\rangle=0(x \in F)\right\}, \quad{ }^{\perp} G=\{x \in E:\langle\mu, x\rangle=0(\mu \in G)\} .
$$


It is then standard that, when $F$ is closed, $F^{\prime}$ is isometrically isomorphic to $E^{\prime} / F^{\perp}$, while $(E / F)^{\prime}$ is isometrically isomorphic to $F^{\perp}$. The weak ${ }^{*}$-closure of $G$ in $E^{\prime}$ is $\left({ }^{\perp} G\right)^{\perp}$.

Proposition 2.4. Let $\mathcal{A}$ be a Banach algebra, and let $X \subseteq \mathcal{A}^{\prime}$ be a closed submodule. Then the following are equivalent:

(1) the first Arens product drops to a well-defined product on $X^{\prime}=$ $\mathcal{A}^{\prime \prime} / X^{\perp}$ turning $\left(X^{\prime}, X\right)$ into a dual Banach algebra;

(2) $X \subseteq \operatorname{WAP}\left(\mathcal{A}^{\prime}\right)$.

Furthermore, let $\mathfrak{A}$ be a subalgebra of $\mathfrak{Z}_{\mathrm{t}}^{(1)}\left(\mathcal{A}^{\prime \prime}\right) \cap \mathfrak{Z}_{\mathrm{t}}^{(2)}\left(\mathcal{A}^{\prime \prime}\right)$, and suppose that the natural map $\mathfrak{A} \rightarrow X^{\prime}$ is surjective. Then (1) and (2) hold, and the algebra product given by (1) agrees with the product induced by the map $\mathfrak{A} \rightarrow X^{\prime}$.

Proof. For $\Phi \in \mathcal{A}^{\prime \prime}$ and $\mu \in X$, suppose that $\Psi \in \mathcal{A}^{\prime \prime}$ is such that $\Phi+X^{\perp}=\Psi+X^{\perp}$, so that

$$
\langle\Phi \cdot \mu, a\rangle=\langle\Phi, \mu \cdot a\rangle=\langle\Psi, \mu \cdot a\rangle=\langle\Psi \cdot \mu, a\rangle \quad(a \in \mathcal{A}),
$$

as $X$ is a submodule. Hence there is a well-defined map $\left(\mathcal{A}^{\prime \prime} / X^{\perp}\right) \times \mathcal{A}^{\prime} \rightarrow \mathcal{A}^{\prime}$ given by $\left(\Phi+X^{\perp}\right) \cdot \mu=\Phi \cdot \mu$, and similarly with orders reversed. It is hence clear that $\square$ gives a well-defined product on $\mathcal{A}^{\prime \prime} / X^{\perp}$ if and only if $\Phi \cdot \mu \in X$ for $\Phi \in \mathcal{A}^{\prime \prime}$ and $\mu \in X$. If this holds, then $X$ is an $X^{\prime}$-module if and only if, for each $\mu \in X$ and $\Phi \in \mathcal{A}^{\prime \prime}$, there exists $\lambda \in X$ such that $\langle\Phi \square \Psi, \mu\rangle=\langle\Psi, \lambda\rangle$ for $\Psi \in \mathcal{A}^{\prime \prime}$. In particular, we see that $\langle\lambda, a\rangle=\langle\Phi \cdot a, \mu\rangle=\langle\mu \cdot \Phi, a\rangle$ for $a \in \mathcal{A}$, that is, $\lambda=\mu \cdot \Phi$, and so we conclude that $\langle\Phi \square \Psi, \mu\rangle=\langle\Phi \diamond \Psi, \mu\rangle$ for $\Phi, \Psi \in \mathcal{A}^{\prime \prime}, \mu \in X$, which is equivalent to $X \subseteq \operatorname{WAP}\left(\mathcal{A}^{\prime}\right)$. Conversely, if (2) holds, then for $\Phi \in \mathcal{A}^{\prime \prime}, \mu \in X$ and $\Psi \in X^{\perp}$, we have $\langle\Psi, \Phi \cdot \mu\rangle=$ $\langle\Psi \diamond \Phi, \mu\rangle=\langle\Phi, \mu \cdot \Psi\rangle=0$ as $\mu \cdot \Psi=0$, which implies that $\Phi \cdot \mu \in X$. Hence conditions (1) and (2) are equivalent.

If $\mathfrak{A} \rightarrow X^{\prime}$ is surjective, then for $\Phi, \Psi \in \mathcal{A}^{\prime \prime}$, we can choose $a, b \in \mathfrak{A}$ such that $a+X^{\perp}=\Phi+X^{\perp}$ and $b+X^{\perp}=\Psi+X^{\perp}$. Then, for $\mu \in X$,

$$
\begin{aligned}
\langle\Phi \square \Psi, \mu\rangle & =\langle\Phi, \Psi \cdot \mu\rangle=\langle\Phi, b \cdot \mu\rangle=\langle\Phi \square b, \mu\rangle=\langle\Phi \diamond b, \mu\rangle=\langle b, \mu \cdot \Phi\rangle \\
& =\langle b, \mu \cdot a\rangle=\langle a \diamond b, \mu\rangle=\langle a \square b, \mu\rangle,
\end{aligned}
$$

where we use the fact that $b \in \mathfrak{Z}_{\mathrm{t}}^{(2)}\left(\mathcal{A}^{\prime \prime}\right)$. Thus the map $\mathfrak{A} \rightarrow X^{\prime}$ gives a well-defined product on $X^{\prime}$. We also see that

$$
\begin{aligned}
\langle a b, \mu\rangle & =\langle a, b \cdot \mu\rangle=\langle a, \Psi \cdot \mu\rangle=\langle a \square \Psi, \mu\rangle=\langle a \diamond \Psi, \mu\rangle \\
& =\langle\Psi, \mu \cdot a\rangle=\langle\Psi, \mu \cdot \Phi\rangle=\langle\Phi \diamond \Psi, \mu\rangle,
\end{aligned}
$$

as $a \in \mathfrak{Z}_{\mathrm{t}}^{(1)}\left(\mathcal{A}^{\prime \prime}\right)$. Hence $\mu \in \operatorname{WAP}\left(\mathcal{A}^{\prime}\right)$, and (2) holds, as required.

We note that in [20, Proposition 4.9], Kaijser explores similar ideas to the above proposition. Furthermore, the equivalence of (1) and (2) is established in [22, Lemma 1.4] in the case of commutative Banach algebras. 
Corollary 2.5. Let $\mathcal{A}$ be a Banach algebra. Then $\operatorname{WAP}\left(\mathcal{A}^{\prime}\right)^{\prime}$ is a dual Banach algebra. Let $\mathcal{A}_{*} \subseteq \mathcal{A}^{\prime}$ be a closed submodule such that, if $\pi: \mathcal{A}^{\prime \prime} \rightarrow$ $\mathcal{A}^{\prime \prime} / \mathcal{A}_{*}^{\perp}=\mathcal{A}_{*}^{\prime}$ is the quotient map, then $\pi \circ \kappa_{\mathcal{A}}: \mathcal{A} \rightarrow \mathcal{A}_{*}^{\prime}$ is an isomorphism. Then $\mathcal{A}_{*}^{\prime}$ is a dual Banach algebra.

Conversely, suppose that $\left(\mathcal{A}, \mathcal{A}_{*}\right)$ is a dual Banach algebra. Then it is a simple calculation (see [26]) that $\kappa_{\mathcal{A}_{*}}^{\prime}: \mathcal{A}^{\prime \prime} \rightarrow \mathcal{A}$ is an algebra homomorphism for either Arens product. Hence we may (and shall) make the following equivalent definition:

Definition 2.6. Let $\mathcal{A}$ be a Banach algebra, let $\mathcal{A}_{*}$ be a closed submodule of $\mathcal{A}^{\prime}$, and let $\pi_{\mathcal{A}_{*}}: \mathcal{A}^{\prime \prime} \rightarrow \mathcal{A}^{\prime \prime} / \mathcal{A}_{*}^{\perp}=\mathcal{A}_{*}^{\prime}$ be the quotient map. When $\pi_{\mathcal{A}_{*}} \circ \kappa_{\mathcal{A}}: \mathcal{A} \rightarrow \mathcal{A}_{*}^{\prime}$ is an isomorphism, we say that $\mathcal{A}$ is a dual Banach algebra with predual $\mathcal{A}_{*}$.

Lemma 2.7. Let $\mathcal{A}$ be a Banach algebra, and let $\mathcal{B}=\operatorname{WAP}\left(\mathcal{A}^{\prime}\right)^{\prime}$. Then $\mathcal{B}$ is unital if and only if there exists $\Phi \in \mathcal{A}^{\prime \prime}$ with $\Phi \cdot \mu=\mu \cdot \Phi=\mu$ for each $\mu \in \operatorname{WAP}\left(\mathcal{A}^{\prime}\right)$. When $\left(\mathcal{A}, \mathcal{A}_{*}\right)$ is a dual Banach algebra, $\mathcal{B}$ is unital if and only if $\mathcal{A}$ is unital.

Proof. Let $e_{\mathcal{B}}$ be the unit of $\mathcal{B}$, so that for some $\Phi \in \mathcal{A}^{\prime \prime}$, we have $\langle\Phi, \mu\rangle=$ $\left\langle e_{\mathcal{B}}, \mu\right\rangle$ for $\mu \in \operatorname{WAP}\left(\mathcal{A}^{\prime}\right)$. Thus, for $\Psi \in \mathcal{A}^{\prime \prime}$,

$$
\langle\Psi, \mu\rangle=\langle\Psi \square \Phi, \mu\rangle=\langle\Psi, \Phi \cdot \mu\rangle=\langle\Phi \diamond \Psi, \mu\rangle=\langle\Psi, \mu \cdot \Phi\rangle \quad\left(\mu \in \operatorname{WAP}\left(\mathcal{A}^{\prime}\right)\right),
$$

as required.

Now suppose that $\mathcal{A}$ is a dual Banach algebra, and let $\left(a_{\alpha}\right)$ be a bounded net in $\mathcal{A}$ tending to $\Phi \in \mathcal{A}^{\prime \prime}$ in the weak*-topology. Let $e \in \mathcal{A}$ be a weak*-limit point of $\left(a_{\alpha}\right)$. Then, for $a \in \mathcal{A}$ and $\mu \in \mathcal{A}_{*} \subseteq \operatorname{WAP}\left(\mathcal{A}^{\prime}\right)$,

$$
\langle a e, \mu\rangle=\langle e, \mu \cdot a\rangle=\lim _{\alpha}\left\langle a_{\alpha}, \mu \cdot a\right\rangle=\langle\Phi, \mu \cdot a\rangle=\langle a, \mu\rangle,
$$

so that $a e=a$. Similarly, $e a=a$, so that $e$ is a unit for $\mathcal{A}$.

Thus looking at $\operatorname{WAP}\left(\mathcal{A}^{\prime}\right)^{\prime}$ is not useful for unitising a dual Banach algebra; instead, $\operatorname{WAP}\left(\mathcal{A}^{\prime}\right)^{\prime}$ is a useful way for embedding a Banach algebra in a dual Banach algebra.

LEMMA 2.8. Let $\mathcal{A}$ and $\mathcal{B}$ be Banach algebras, and let $\pi: \mathcal{A} \rightarrow \mathcal{B}$ be a homomorphism. Then $\pi^{\prime}\left(\operatorname{WAP}\left(\mathcal{B}^{\prime}\right)\right) \subseteq \operatorname{WAP}\left(\mathcal{A}^{\prime}\right)$.

Proof. Let $\mu \in \operatorname{WAP}\left(\mathcal{B}^{\prime}\right)$, and let $\lambda=\pi^{\prime}(\mu) \in \mathcal{A}^{\prime}$, so that

$$
\left\langle L_{\lambda}(a), b\right\rangle=\langle\lambda \cdot a, b\rangle=\langle\mu, \pi(a b)\rangle=\langle\mu \cdot \pi(a), \pi(b)\rangle=\left\langle\pi^{\prime} L_{\mu} \pi(a), b\right\rangle .
$$

So $L_{\lambda}=\pi^{\prime} \circ L_{\mu} \circ \pi$ is weakly compact, as $L_{\mu}$ is weakly compact.

Weak ${ }^{*}$-continuous representations of $\operatorname{WAP}\left(\mathcal{A}^{\prime}\right)^{\prime}$ are closely related to continuous representations of $\mathcal{A}$, a fact first noted by Runde. The following is [28, Theorem 4.10]. 
Proposition 2.9. Let $\mathcal{A}$ be a Banach algebra, let $\left(\mathcal{B}, \mathcal{B}_{*}\right)$ be a dual Banach algebra, and let $\pi: \mathcal{A} \rightarrow \mathcal{B}$ be a homomorphism. Then there is a unique weak $k^{*}$-continuous homomorphism $\widehat{\pi}: \operatorname{WAP}\left(\mathcal{A}^{\prime}\right)^{\prime} \rightarrow \mathcal{B}$ such that $\widehat{\pi} \circ \kappa_{\mathcal{A}}=\pi$. In particular, a weak $k^{*}$-continuous homomorphism $\theta: \operatorname{WAP}\left(\mathcal{A}^{\prime}\right)^{\prime} \rightarrow \mathcal{B}$ is uniquely determined by its restriction to $\mathcal{A}$.

Definition 2.10. Let $\mathcal{A}$ be a Banach algebra. We call $\operatorname{WAP}\left(\mathcal{A}^{\prime}\right)^{\prime}$ the dual Banach algebra $(D B A)$ enveloping algebra of $\mathcal{A}$. When the natural map of $\mathcal{A}$ into $\operatorname{WAP}\left(\mathcal{A}^{\prime}\right)^{\prime}$ is bounded below, we say that $\mathcal{A}$ is a WAP algebra.

We note that every dual Banach algebra, and every Arens regular Banach algebra, is a WAP algebra. We shall shortly see that group algebras are also always WAP algebras, even in the non-discrete case, in which case they are neither dual Banach algebras, nor Arens regular. The above proposition shows us that the weak ${ }^{*}$-continuous theory of the DBA enveloping algebra is determined by $\mathcal{A}$. We see from this, and from later results, that the DBA enveloping algebra plays much the same role as the enveloping $\mathrm{W}^{*}$-algebra of a $\mathrm{C}^{*}$-algebra does (see [32, Chapter III, Section 2]).

3. Representations for WAP algebras. We have already noted that work of Young shows that a WAP algebra $\mathcal{A}$ admits a representation $\pi: \mathcal{A} \rightarrow$ $\mathcal{B}(E)$ for some reflexive Banach space $E$, such that $\pi$ is bounded below. In fact, Young effectively shows that $\mu \in \operatorname{WAP}\left(\mathcal{A}^{\prime}\right)$ if and only if there exists a reflexive Banach space $E$, a representation $\pi: \mathcal{A} \rightarrow \mathcal{B}(E), x \in E$ and $\lambda \in E^{\prime}$ with $\|x\|\|\lambda\|=\|\mu\|$ and such that $\pi^{\prime} \kappa_{E^{\prime} \widehat{\otimes} E}(\lambda \otimes x)=\mu$. In particular, we see that $\pi^{\prime} \kappa_{E^{\prime} \widehat{\otimes} E}$ maps $E^{\prime} \widehat{\otimes} E$ onto $\operatorname{WAP}\left(\mathcal{A}^{\prime}\right)$.

In this section, we shall use some interpolation space theory to prove an analogous result for dual Banach algebras which does not seem to immediately follow from the results of Young (although the method of proof is much the same). We shall later use interpolation space theory for other reasons, so it is useful to define some concepts now. Interpolation space arguments in this area go back to [10]; we follow the text [3] for results on interpolation spaces.

Definition 3.1. Let $\left(\mathcal{A}, \mathcal{A}_{*}\right)$ be a dual Banach algebra, and let $\mu \in \mathcal{A}_{*}$. Suppose that there exists a norm $\|\cdot\|_{\mu}$ on $\mathcal{A} \cdot \mu=\{a \cdot \mu: a \in \mathcal{A}\}$ such that the completion of $\left(\mathcal{A} \cdot \mu,\|\cdot\|_{\mu}\right)$, denoted by $E_{\mu}$, is reflexive, and such that

$$
\|a b \cdot \mu\|_{\mu} \leq\|a\|\|b \cdot \mu\|_{\mu}, \quad\|a \cdot \mu\| \leq\|a \cdot \mu\|_{\mu} \leq\|a\|\|\mu\| \quad(a, b \in \mathcal{A}) .
$$

Let $\iota: E_{\mu} \rightarrow \mathcal{A}_{*}$ be the norm-decreasing inclusion map, and suppose further that $\iota$ is injective. Then we say that $\|\cdot\|_{\mu}$ is an admissible norm for $\mu$.

EXAmple 3.2 . Let $\left(\mathcal{A}, \mathcal{A}_{*}\right)$ be a $\mathrm{W}^{*}$-algebra, and let $\mu \in \mathcal{A}_{*}$ be a state. Then it is simple to check that the usual GNS construction for $\mu$ (see [32, Chapter I, Section 9]) induces an admissible norm on $\mathcal{A} \cdot \mu$. 
EXAmPle 3.3. We note that $\|\cdot\|_{\mu}$ need not be unique (even in an isomorphic sense). For example, let $\mathcal{A}=\ell^{2}(\mathbb{N})$ with pointwise multiplication, and let $\mu \in \mathcal{A}^{\prime}=\ell^{2}$ be such that the map $\mathcal{A} \rightarrow \mathcal{A}^{\prime}, a \mapsto a \cdot \mu$, is injective and $\|\mu\|=1$ (for example, $\left.\mu=\left(2^{-n / 2}\right)_{n>0} \in \ell^{2}\right)$. Then define

$$
\|a \cdot \mu\|_{\mu, 1}=\|a \cdot \mu\|, \quad\|a \cdot \mu\|_{\mu, 2}=\|a\| \quad(a \in \mathcal{A}),
$$

and let $E_{\mu, 1}$ and $E_{\mu, 2}$ be associated with $\|\cdot\|_{\mu, 1}$ and $\|\cdot\|_{\mu, 2}$ respectively. Then $E_{\mu, 1}$ is the closure of $\mathcal{A} \cdot \mu$ in $\ell^{2}$, while $E_{\mu, 2}=\mathcal{A}$, which are both reflexive, as $\ell^{2}$ is reflexive. We then check that

$$
\begin{aligned}
\|a b \cdot \mu\|_{\mu, 1} & =\sup \{|\langle a b \cdot \mu, c\rangle|:\|c\| \leq 1\}=\sup \{|\langle b \cdot \mu, d\rangle|: d=c a,\|c\| \leq 1\} \\
& \leq \sup \{|\langle b \cdot \mu, d\rangle|:\|d\| \leq\|a\|\}=\|a\|\|b \cdot \mu\|=\|a\|\|b \cdot \mu\|_{\mu, 1},
\end{aligned}
$$

while clearly $\|a b \cdot \mu\|_{\mu, 2} \leq\|a\|\|b \cdot \mu\|_{\mu, 2}$. Hence both $\|\cdot\|_{\mu, 1}$ and $\|\cdot\|_{\mu, 2}$ are admissible, but clearly they are not equivalent norms.

Lemma 3.4. Let $\left(\mathcal{A}, \mathcal{A}_{*}\right)$ be a dual Banach algebra, let $\mu \in \mathcal{A}_{*}$ have an admissible norm, and let $E_{\mu}$ be a space as defined above using some admissible norm for $\mu$. Then $\iota^{\prime}: \mathcal{A} \rightarrow E_{\mu}^{\prime}$ has dense range, the module action of $\mathcal{A}$ on $E_{\mu}$ induces a weak ${ }^{*}$-continuous representation $\mathcal{A} \rightarrow \mathcal{B}\left(E_{\mu}\right)$, and there exist $x \in E_{\mu}$ and $\lambda \in E_{\mu}^{\prime}$ such that $\|x\|\|\lambda\|=\|\mu\|$ and $\langle\lambda, a \cdot x\rangle=\langle a, \mu\rangle$ for $a \in \mathcal{A}$.

Proof. We may suppose that $\|\mu\|=1$. By the condition on $\|\cdot\|_{\mu}$, we see that $\iota$ is norm-decreasing, and the module action inherited from $\mathcal{A}_{*}$ induces a Banach left $\mathcal{A}$-module action on $E_{\mu}$. Furthermore, $\iota^{\prime}$ has dense range if and only if $\iota^{\prime \prime}: E_{\mu}^{\prime \prime} \rightarrow \mathcal{A}^{\prime}$ is injective, which, as $E_{\mu}$ is reflexive, is in turn equivalent to $\iota$ being injective. We define $\psi_{\mu}: E_{\mu}^{\prime} \widehat{\otimes} E_{\mu} \rightarrow \mathcal{A}_{*}$ by

$$
\psi_{\mu}\left(\iota^{\prime}(a) \otimes b \cdot \mu\right)=b \cdot \mu \cdot a \quad\left(a \in \mathcal{A}, b \cdot \mu \in E_{\mu}\right) .
$$

Assuming this is bounded, $\psi_{\mu}$ extends by linearity and continuity to $E_{\mu}^{\prime} \widehat{\otimes} E_{\mu}$. Indeed, we have

$$
\begin{aligned}
\|b \cdot \mu \cdot a\| & =\sup \{|\langle a c b, \mu\rangle|:\|c\| \leq 1\}=\sup \{|\langle a, c b \cdot \mu\rangle|:\|c\| \leq 1\} \\
& \leq \sup \left\{|\langle a, d \cdot \mu\rangle|:\|d \cdot \mu\|_{E_{\mu}} \leq\|b \cdot \mu\|_{E_{\mu}}\right\}=\left\|\iota^{\prime}(a)\right\|_{E_{\mu}^{\prime}}\|b \cdot \mu\|_{E_{\mu}},
\end{aligned}
$$

as $\|c b \cdot \mu\|_{E_{\mu}} \leq\|c\|\|b \cdot \mu\|_{E_{\mu}}$. Thus $\psi_{\mu}$ is norm-decreasing. Then let $\theta_{\mu}=\psi_{\mu}^{\prime}$ : $\mathcal{A} \rightarrow \mathcal{B}\left(E_{\mu}\right)$, so that for $a, b, c \in \mathcal{A}$,

$$
\left\langle\iota^{\prime}(b), \theta_{\mu}(a)(c \cdot \mu)\right\rangle=\left\langle a, \psi_{\mu}\left(\iota^{\prime}(b) \otimes c \cdot \mu\right)\right\rangle=\langle b, a c \cdot \mu\rangle=\left\langle\iota^{\prime}(b), a \cdot(c \cdot \mu)\right\rangle ;
$$

hence $\theta_{\mu}$ agrees with the left-module action of $\mathcal{A}$ on $E_{\mu}$, as required.

Finally, we see that $\psi_{\mu}\left(\iota^{\prime}\left(e_{\mathcal{A}}\right) \otimes e_{\mathcal{A}} \cdot \mu\right)=\mu$, where

$$
\begin{aligned}
\left\|\iota^{\prime}\left(e_{\mathcal{A}}\right)\right\|_{E_{\mu}^{\prime}} & =\sup \left\{\left|\left\langle e_{\mathcal{A}}, a \cdot \mu\right\rangle\right|:\|a \cdot \mu\|_{E_{\mu}} \leq 1\right\} \\
& \leq \sup \left\{\left|\left\langle e_{\mathcal{A}}, a \cdot \mu\right\rangle\right|:\|a \cdot \mu\| \leq 1\right\} \leq\left\|e_{\mathcal{A}}\right\|=1,
\end{aligned}
$$

and $\left\|e_{\mathcal{A}} \cdot \mu\right\|_{E_{\mu}} \leq\left\|e_{\mathcal{A}}\right\|\|\mu\|=1$. 
TheOREM 3.5. Let $\left(\mathcal{A}, \mathcal{A}_{*}\right)$ be a dual Banach algebra such that each norm-one member of $\mathcal{A}_{*}$ has an admissible norm. Then $\mathcal{A}$ is isometric, via a weak*-weak*-continuous map, to a weak $k^{*}$-closed subalgebra of $\mathcal{B}(E)$ for some reflexive Banach space $E$.

Proof. We may suppose that $\mathcal{A}$ is unital, as otherwise we may work with $\left(\mathcal{A}, \mathcal{A}_{*}\right)^{\sharp}$, and then restrict the resulting representation to $\mathcal{A}$. Let $X=$ $\left\{\mu \in \mathcal{A}_{*}:\|\mu\|=1\right\}$, and let $E=\ell^{2}\left(\bigoplus_{\mu \in X} E_{\mu}\right)$, so that $E$ is a reflexive Banach space. Define $\psi: E^{\prime} \widehat{\otimes} E \rightarrow \mathcal{A}_{*}$ by

$$
\psi\left(\left(\lambda_{\mu}\right) \otimes\left(x_{\mu}\right)\right)=\sum_{\mu \in X} \psi_{\mu}\left(\lambda_{\mu} \otimes x_{\mu}\right) \quad\left(\left(\lambda_{\mu}\right) \in E^{\prime},\left(x_{\mu}\right) \in E\right) .
$$

This is norm-decreasing, as

$$
\left\|\sum_{\mu \in X} \psi_{\mu}\left(\lambda_{\mu} \otimes x_{\mu}\right)\right\| \leq \sum_{\mu \in X}\left\|\lambda_{\mu}\right\|\left\|x_{\mu}\right\| \leq\left(\sum_{\mu \in X}\left\|\lambda_{\mu}\right\|^{2}\right)^{1 / 2}\left(\sum_{\mu \in X}\left\|x_{\mu}\right\|^{2}\right)^{1 / 2} .
$$

Then let $\theta=\psi^{\prime}: \mathcal{A} \rightarrow \mathcal{B}(E)$, so that $\theta$ is weak*-continuous, and for $a, b \in \mathcal{A}$, $\left(x_{\mu}\right) \in E$ and $\left(\lambda_{\mu}\right) \in E^{\prime}$, we have

$$
\left\langle\left(\lambda_{\mu}\right), \theta(b)\left(x_{\mu}\right)\right\rangle=\sum_{\mu \in X}\left\langle b, \psi_{\mu}\left(\lambda_{\mu} \otimes x_{\mu}\right)\right\rangle=\sum_{\mu \in X}\left\langle\lambda_{\mu}, \theta_{\mu}(b)\left(x_{\mu}\right)\right\rangle ;
$$

therefore $\theta(b)\left(x_{\mu}\right)=\left(\theta_{\mu}(b)\left(x_{\mu}\right)\right)$, and so $\theta$ is a homomorphism, as each $\theta_{\mu}$ is a homomorphism.

It is a standard result that $\theta=\psi^{\prime}$ has a weak ${ }^{*}$-closed image if and only if $\theta$ has a closed image, which happens if and only if $\theta$ is bounded below. For $a \in \mathcal{A}$ and $\varepsilon>0$, there exists $\mu \in X$ such that $|\langle a, \mu\rangle|>(1-\varepsilon)\|a\|$. Then $\mu=\psi\left(\lambda_{\mu} \otimes x_{\mu}\right)$ for some $\lambda_{\mu} \in E_{\mu}^{\prime}$ and $x_{\mu} \in E_{\mu}$ with $\left\|\lambda_{\mu}\right\|\left\|x_{\mu}\right\|=1$. Thus

$$
\|\theta(a)\| \geq\left\|\theta_{\mu}(a)\right\| \geq\left|\left\langle a, \psi\left(\lambda_{\mu} \otimes x_{\mu}\right)\right\rangle\right|>(1-\varepsilon)\|a\| .
$$

As $\varepsilon>0$ was arbitrary, we see that $\theta$ is an isometry onto its range.

Of course, we have not shown that any dual Banach algebra (other than a $\mathrm{W}^{*}$-algebra) admits such a representation. We now remedy this situation by using some interpolation space theory.

Theorem 3.6. Let $\mathcal{A}$ be a unital dual Banach algebra with predual $\mathcal{A}_{*}$. Then each norm-one member of $\mathcal{A}_{*}$ has an admissible norm.

Proof. Let $\mu \in \mathcal{A}_{*}$ be such that $\|\mu\|=1$, and for $n \in \mathbb{N}$ define a new norm on $\mathcal{A}^{\prime}$ by

$$
\|\lambda\|_{n}=\inf \left\{2^{-n / 2}\|b\|+2^{n / 2}\|\lambda-b \cdot \mu\|: b \in \mathcal{A}\right\} \quad\left(\lambda \in \mathcal{A}^{\prime}\right) .
$$

Then, for $a, b \in \mathcal{A}$ and $\lambda \in \mathcal{A}^{\prime}$, we have $\|\lambda\|_{n} \leq 2^{n / 2}\|\lambda\|,\|a \cdot \mu\|_{n} \leq 2^{-n / 2}\|a\|$, and $\|\lambda\| \leq\|\lambda-b \cdot \mu\|+\|b \cdot \mu\| \leq 2^{n}\|\lambda-b \cdot \mu\|+\|b\|$ so that $2^{-n / 2}\|\lambda\| \leq\|\lambda\|_{n}$. 
We then define

$$
E_{\mu}=\left\{\lambda \in \mathcal{A}^{\prime}:\|\lambda\|_{\mu}:=\left(\sum_{n=1}^{\infty}\|\lambda\|_{n}^{2}\right)^{1 / 2}<\infty\right\} .
$$

If $\lambda \in E_{\mu}$, then there exists some sequence $\left(b_{n}\right)$ in $\mathcal{A}$ with $2^{n}\left\|\lambda-b_{n} \cdot \mu\right\|^{2} \rightarrow 0$ as $n \rightarrow \infty$. In particular, $\mathcal{A} \cdot \mu$ is dense in $E_{\mu}$. Thus, for $a \in \mathcal{A}$, we have $\|a \cdot \mu\| \leq\|a \cdot \mu\|_{\mu} \leq\|a\|$. We can also easily check that $\|a \cdot \lambda\|_{\mu} \leq\|a\|\|\lambda\|_{\mu}$ for $a \in \mathcal{A}$ and $\lambda \in E_{\mu}$. Hence we need only show that $E_{\mu}$ is reflexive to verify the conditions of Definition 3.1.

Recall that $R_{\mu}: \mathcal{A} \rightarrow \mathcal{A}^{\prime}$ is defined by $R_{\mu}(a)=a \cdot \mu$. Then $R_{\mu}$ maps into $\mathcal{A}_{*}$ and is weakly compact, as $\mathcal{A}_{*} \subseteq \operatorname{WAP}\left(\mathcal{A}^{\prime}\right)$. It follows from the work in [10] (see [25, Section 1.7.8] for a sketch) that $E_{\mu}$ is reflexive, as the map $R_{\mu}$ is weakly compact.

Notice that the above proof will work for any $\mu \in \operatorname{WAP}\left(\mathcal{A}^{\prime}\right)$, which re-creates Young's result.

REMARK 3.7. The above construction of $E_{\mu}$ is actually a Lions-Peetre interpolation space. Let $\mathcal{A} \cdot \mu$ be the subspace of $\mathcal{A}_{*}$ spanned by $\{a \cdot \mu: a \in \mathcal{A}\}$ together with the norm $\|a \cdot \mu\|_{\mathcal{A} \cdot \mu}=\inf \{\|b\|: b \cdot \mu=a \cdot \mu\}$. Then we see that $R_{\mu}: \mathcal{A} \rightarrow \mathcal{A} \cdot \mu$ is norm-decreasing, and the induced map $\mathcal{A} /$ ker $R_{\mu} \rightarrow \mathcal{A} \cdot \mu$ is an isometry, showing that $\mathcal{A} \cdot \mu$ is a Banach space.

Following [3], we let $\mathcal{S}\left(\mathcal{A} \cdot \mu, \mathcal{A}_{*}\right)$ be the space $\mathcal{A}_{*}$ together with the norm

$$
\|\lambda\|_{\mathcal{S}}=\inf \left\{\|a \cdot \mu\|_{\mathcal{A} \cdot \mu}+\|\phi\|: \lambda=a \cdot \mu+\phi\right\} \quad\left(\lambda \in \mathcal{A}_{*}\right) .
$$

Then $\mathcal{S}$ is a Banach space. Let $1 \leq p<\infty, \xi_{0}<0$ and $\xi_{1}>0$, and let $s_{2}^{+}\left(p ; \xi_{0}, \mathcal{A} \cdot \mu ; \xi_{1}, \mathcal{A}_{*}\right)$ be the subspace of $\mathcal{S}$ such that

$$
\begin{array}{r}
\|\lambda\|_{s_{2}^{+}}=\inf \left\{\max \left(\left(\sum_{n=1}^{\infty}\left\|e^{\xi_{0} n} a_{n} \cdot \mu\right\|_{\mathcal{A} \cdot \mu}^{p}\right)^{1 / p},\left(\sum_{n=1}^{\infty}\left\|e^{\xi_{1} n} \phi_{n}\right\|^{p}\right)^{1 / p}\right):\right. \\
\left.\lambda=a_{n} \cdot \mu+\phi_{n}(n>0)\right\}<\infty .
\end{array}
$$

In comparison, we see that $E_{\mu}$ is the subspace of $\mathcal{S}$ such that

$$
\|\lambda\|_{\mu}=\inf \left\{\left(\sum_{n=1}^{\infty}\left(2^{-n / 2}\left\|a_{n} \cdot \mu\right\|_{\mathcal{A} \cdot \mu}+2^{n / 2}\left\|\phi_{n}\right\|\right)^{2}\right)^{1 / 2}: \lambda=a_{n} \cdot \mu+\phi_{n}(n>0)\right\} .
$$

We thus see that $s_{2}^{+}\left(2 ;-\log \sqrt{2}, \mathcal{A} \cdot \mu ; \log \sqrt{2}, \mathcal{A}_{*}\right)$ is isomorphic to $E_{\mu}$.

It follows from [3, Section 1.5, Proposition 1] that $E_{\mu}$ is a member of the isomorphic class $\left(\mathcal{A} \cdot \mu, \mathcal{A}_{*}\right)_{1 / 2,2}$, and hence [3, Section 2.3, Proposition 1] tells us that $E_{\mu}$ is reflexive if and only if the inclusion $\mathcal{A} \cdot \mu \rightarrow \mathcal{A}_{*}$ is weakly compact, which happens if and only if $R_{\mu}$ is weakly compact, as before.

Putting all these results together, we obtain the following. 
Corollary 3.8. Let $\left(\mathcal{A}, \mathcal{A}_{*}\right)$ be a dual Banach algebra. Then $\mathcal{A}$ admits an isometric, weak $k^{*}$-weak $k^{*}$-continuous representation on some reflexive $B a$ nach space.

Proposition 3.9. Let $\left(\mathcal{A}, \mathcal{A}_{*}\right)$ be a $W^{*}$-algebra (that is, $\mathcal{A}$ is a $C^{*}$-algebra), and form $E$ as above using only the $\mu \in \mathcal{A}_{*}$ which are states. Then $E$ is isomorphic to a Hilbert space, and our representation agrees with the usual universal representation for a $W^{*}$-algebra.

Proof. This follows from work in [12].

4. Unique preduals. It is a standard result in the theory of $\mathrm{W}^{*}$-algebras that a $\mathrm{W}^{*}$-algebra has a unique predual, up to isometric classification. That is, if $\left(\mathcal{A}, \mathcal{A}_{*}\right)$ is a $\mathrm{W}^{*}$-algebra, $E$ is a Banach space, and $\theta: \mathcal{A} \rightarrow E^{\prime}$ is an isometric isomorphism, then $\theta$ is automatically weak*-continuous. This follows as we can use $\theta$ to induce a $\mathrm{C}^{*}$-algebra structure on $E^{\prime}$, showing that $E$ is also a predual for $\mathcal{A}$.

The theory of isometric preduals in Banach spaces has attracted some attention (see the survey [15]). However, here we are interested in the isomorphic and not isometric theory.

Theorem 4.1. Let $\left(\mathcal{A}, \mathcal{A}_{*}\right)$ be a commutative $W^{*}$-algebra, let $\left(\mathcal{B}, \mathcal{B}_{*}\right)$ be a dual Banach algebra, and let $\theta: \mathcal{A} \rightarrow \mathcal{B}$ be a Banach algebra isomorphism. Then $\theta$ is automatically weak*-continuous.

Proof. By [32, Theorem 1.18], $\mathcal{A}$ can be identified with $C(\Omega)$ where $\Omega$ is the character space of $\mathcal{A}$, which is a hyperstonian space (see [2, Section 8] for further details). In particular, $\Omega$ is Stonian in that the closure of any open set is open. Then $\mathcal{A}^{\prime}$ is $M(\Omega)$, the space of regular Borel measures on $\Omega$. We say that a positive measure $\mu \in M(\Omega)$ is normal if $\mu(A)=0$ whenever $A$ is nowhere dense, that is, the closure of $A$ has empty interior. A general measure is normal when its absolute value is normal. By [2, Theorem 8.2] this definition agrees with the usual one for $\mathrm{W}^{*}$-algebras (see also [32, Proposition 1.11]). Then the collection of normal measures forms a closed subspace of $M(\Omega)$ which is equal to $\kappa_{\mathcal{A}_{*}}\left(\mathcal{A}_{*}\right)$.

By reversing the argument which led to Definition 2.6, we need to show that if $E \subseteq \mathcal{A}^{\prime}$ is a closed submodule such that the natural map $\iota_{E}: \mathcal{A} \rightarrow$ $E^{\prime}=\mathcal{A}^{\prime \prime} / E^{\perp}$ is an isomorphism, then $E=\kappa_{\mathcal{A}_{*}}\left(\mathcal{A}_{*}\right)$. The equivalence of this statement to the statement involving $\mathcal{B}$ comes from setting $E=\theta^{\prime} \kappa_{\mathcal{B}_{*}}\left(\mathcal{B}_{*}\right)$.

Let $E$ be as stated, and choose $\lambda \in E$. We will show that $\lambda$ is normal, which will complete the proof, as then $E \subseteq \mathcal{A}_{*}$, and so necessarily $E=\mathcal{A}_{*}$. Let $A$ be a closed subset of $\Omega$ with empty interior, so we aim to show that $|\lambda|(A)=0$. Consider the family $\mathcal{C}$ of closed and open subsets of $\Omega$ which contain $A$, partially ordered by reverse inclusion. For $B \in \mathcal{C}$, let $\chi_{B}$ be the 
indicator function of $B$, so that $\chi_{B} \in C(\Omega)$. As $E$ is a predual, there exists a unique $f \in C(\Omega)$ such that

$$
\langle\mu, f\rangle=\lim _{B \in \mathcal{C}}\left\langle\mu, \chi_{B}\right\rangle \quad(\mu \in E) .
$$

For $C \in \mathcal{C}$, notice that

$$
\left\langle\mu, \chi_{C} f\right\rangle=\left\langle\mu \cdot \chi_{C}, f\right\rangle=\lim _{B \in \mathcal{C}}\left\langle\mu \cdot \chi_{C}, \chi_{B}\right\rangle=\lim _{B \in \mathcal{C}}\left\langle\mu, \chi_{B}\right\rangle=\langle\mu, f\rangle \quad(\mu \in E),
$$

so that $\chi_{C} f=f$, and hence, for $x \in \Omega \backslash C, f(x)=f(x) \chi_{C}(x)=0$. We hence see that $f$ vanishes off the set $A_{0}:=\bigcap_{B \in \mathcal{C}} B$.

We claim that $A_{0}=A$, which follows from some simple topology. Indeed, clearly $A \subseteq A_{0}$, and suppose towards a contradiction that there exists $x \in$ $A_{0} \backslash A$. Thus, for each open $B \subseteq \Omega$ with $A \subseteq \bar{B}$, we have $x \in \bar{B}$. As $\Omega$ is Hausdorff, for each $a \in A$ there exist disjoint open sets $U_{a}$ and $V_{a}$ with $a \in U_{a}$ and $x \in V_{a}$. As $A$ is compact, there exist $a_{1}, \ldots, a_{n}$ in $A$ such that $A \subseteq U:=U_{a_{1}} \cup \cdots \cup U_{a_{n}}$. Clearly $U$ is disjoint from the open set $V:=V_{a_{1}} \cap \cdots \cap V_{a_{n}}$, hence as $x \in V$, we see that $x \notin \bar{U}$, a contradiction.

Consequently, $f$ is supported on $A$, and as $A$ has empty interior and $f$ is continuous, we must have $f=0$. Let $\lambda=\lambda_{r}+i \lambda_{i}$ where $\lambda_{r}$ and $\lambda_{i}$ are real measures. Then, as $\lim _{B \in \mathcal{C}}\left\langle\lambda, \chi_{B}\right\rangle=0$, we see that

$$
\lim _{B \in \mathcal{C}}\left\langle\lambda_{r}, \chi_{B}\right\rangle=\lim _{B \in \mathcal{C}}\left\langle\lambda_{i}, \chi_{B}\right\rangle=0,
$$

as $\chi_{B}$ is real-valued. There exists a Hahn decomposition (see [18, Section 29]) for $\lambda_{r}$, that is, measurable sets $E_{+}$and $E_{-}$such that $\Omega=E_{+} \cup E_{-}$and with

$$
\lambda_{+}(E)=\lambda_{r}\left(E \cap E_{+}\right), \quad \lambda_{-}(E)=-\lambda_{r}\left(E \cap E_{-}\right) \quad(E \subseteq \Omega),
$$

defining two positive measures $\lambda_{+}$and $\lambda_{-}$with $\left|\lambda_{r}\right|=\lambda_{+}+\lambda_{-}$and $\lambda_{r}=$ $\lambda_{+}-\lambda_{-}$. As $\left|\lambda_{r}\right|$ is regular, for each $\varepsilon>0$, there exists an open set $U$ and a closed set $K$ such that $K \subseteq E_{+} \subseteq U$ with $\left|\lambda_{r}\right|(U \backslash K)<\varepsilon$. As $\Omega$ is Stonian, we can find an open and closed set $V$ such that $K \subseteq V \subseteq U$ (this follows by a similar argument to that employed above to show that $\left.A_{0}=A\right)$. Then

$$
\left|\lambda_{r}\right|\left(E_{+} \backslash V\right)+\left|\lambda_{r}\right|\left(V \backslash E_{+}\right) \leq\left|\lambda_{r}\right|(U \backslash K)<\varepsilon .
$$

We hence see that for $B \subseteq \Omega$,

$$
\begin{aligned}
\lambda_{+}(B) & =\lambda\left(B \cap E_{+}\right)=\left|\lambda_{r}(B \cap V)+\lambda_{r}\left(B \cap\left(E_{+} \backslash V\right)\right)-\lambda_{r}\left(B \cap\left(V \backslash E_{+}\right)\right)\right| \\
& \leq \varepsilon+\left|\lambda_{r}(B \cap V)\right| .
\end{aligned}
$$

Consequently,

$$
0 \leq \lim _{B \in \mathcal{C}}\left\langle\lambda_{+}, \chi_{B}\right\rangle \leq \varepsilon+\lim _{B \in \mathcal{C}}\left\langle\lambda_{r}, \chi_{V} \chi_{B}\right\rangle=\varepsilon+\lim _{B \in \mathcal{C}}\left\langle\lambda_{r} \cdot \chi_{V}, \chi_{B}\right\rangle=\varepsilon,
$$

so as $\varepsilon>0$ was arbitrary, $\lim _{B \in \mathcal{C}}\left\langle\lambda_{+}, \chi_{B}\right\rangle=0$. We then have

$$
0 \leq \lambda_{+}(A) \leq \inf _{B \in \mathcal{C}} \lambda_{+}(B)=0 .
$$


A similar argument shows that $\lambda_{-}(A)=0$, therefore $\left|\lambda_{r}\right|(A)=0$. Similarly, $\left|\lambda_{i}\right|(A)=0$, so that $|\lambda|(A)=0$. Thus $\lambda$ is normal, as required.

We note that we cannot drop the assumption that $\theta$ is an algebra homomorphism. This follows as, for example, Pełczyński showed in [27] that $\ell^{\infty}$ and $L^{\infty}[0,1]$ are isomorphic (but not isometric, and not isomorphic as Banach algebras in their natural products). However, of course, $\ell^{1}$ and $L^{1}[0,1]$ are not isomorphic, so no isomorphism $\ell^{\infty} \rightarrow L^{\infty}[0,1]$ can be weak*continuous.

Continuing the theme of unique preduals, we have the following, which is [16, Proposition 5.10].

Proposition 4.2. Let $E$ be a reflexive Banach space. Then the predual $E^{\prime} \widehat{\otimes} E$ is isometrically unique for $\mathcal{B}(E)$, meaning that when $\phi: F^{\prime} \rightarrow \mathcal{B}(E)$ is an isometric isomorphism for some Banach space $F$, there exists an isometry $\psi: E^{\prime} \widehat{\otimes} E \rightarrow F$ such that $\psi^{\prime}=\phi$.

Definition 4.3. Let $E$ be a Banach space, and suppose that for each compact set $K \subseteq E$ and each $\varepsilon>0$, there exists a finite-rank operator $T \in \mathcal{F}(E)$ such that $\|T(x)-x\|<\varepsilon$ for each $x \in K$. Then we say that $E$ has the approximation property. When we can choose $T$ to be uniformly bounded, $E$ has the bounded approximation property, and when we can choose $T$ be a contraction, $E$ has the metric approximation property.

See [31, Chapter 4] or [14, Chapter VIII, Section 3] for further details. When $E$ is a reflexive Banach space, the approximation property implies the metric approximation property (see [31, Corollary 5.51]). The approximation property is equivalent to the natural map $E^{\prime} \widehat{\otimes} E \rightarrow \mathcal{B}(E)$ being injective.

THEOREM 4.4. Let $E$ be a reflexive Banach space with the approximation property. Whenever $\left(\mathcal{A}, \mathcal{A}_{*}\right)$ is a dual Banach algebra and $\theta: \mathcal{B}(E) \rightarrow \mathcal{A}$ is a Banach algebra isomorphism, $\theta$ is weak*-continuous.

Proof. Again, we need to show that when $X \subseteq \mathcal{B}(E)^{\prime}$ is a predual, we have $X=\kappa_{E^{\prime} \widehat{\otimes} E}\left(E^{\prime} \widehat{\otimes} E\right)$. As $E$ is reflexive and has the approximation property, $\mathcal{A}(E)^{\prime}=E^{\prime} \widehat{\otimes} E$ and so $\mathcal{A}(E)^{\prime \prime}=\mathcal{B}(E)$. We may check that $\mathcal{A}(E)$ is Arens regular, and that the Arens products on $\mathcal{A}(E)^{\prime \prime}$ agree with the usual product on $\mathcal{B}(E)$. By standard results, as $\mathcal{B}(E)$ is unital, there exists a bounded approximate identity $\left(e_{\alpha}\right)$ for $\mathcal{A}(E)$ (see [8, Proposition 2.9.16] or [25, Section 1.7.13] for further details).

Let $\iota: \mathcal{A}(E) \rightarrow \mathcal{B}(E)$ be the inclusion map, so that actually $\iota$ agrees with the map $\kappa_{\mathcal{A}(E)}$. Then $\iota^{\prime}: \mathcal{B}(E)^{\prime} \rightarrow E^{\prime} \widehat{\otimes} E$ satisfies $\iota^{\prime} \kappa_{E^{\prime} \widehat{\otimes} E}=I_{E^{\prime} \widehat{\otimes} E}$. As $X$ is a predual, there exists a unique $P \in \mathcal{B}(E)$ such that

$$
\langle\mu, P\rangle=\lim _{\alpha}\left\langle\mu, \iota\left(e_{\alpha}\right)\right\rangle=\lim _{\alpha}\left\langle\iota^{\prime}(\mu), e_{\alpha}\right\rangle=\left\langle I_{E}, \iota^{\prime}(\mu)\right\rangle \quad(\mu \in X) .
$$


Then, for $T \in \mathcal{B}(E)$ and $\mu \in X$,

$$
\begin{aligned}
\langle\mu, P T\rangle & =\langle T \cdot \mu, P\rangle=\lim _{\alpha}\left\langle T \cdot \mu, \iota\left(e_{\alpha}\right)\right\rangle=\lim _{\alpha}\left\langle\mu, \iota\left(e_{\alpha} T\right)\right\rangle=\lim _{\alpha}\left\langle\iota^{\prime}(\mu), e_{\alpha} T\right\rangle \\
& =\lim _{\alpha}\left\langle T \cdot \iota^{\prime}(\mu), e_{\alpha}\right\rangle=\left\langle I_{E}, T \cdot \iota^{\prime}(\mu)\right\rangle=\left\langle T, \iota^{\prime}(\mu)\right\rangle
\end{aligned}
$$

and so, for each $\alpha$,

$$
\left\langle\mu, P \iota\left(e_{\alpha}\right)\right\rangle=\left\langle\iota\left(e_{\alpha}\right), \iota^{\prime}(\mu)\right\rangle=\left\langle\mu, \iota\left(e_{\alpha}\right)\right\rangle \quad(\mu \in X),
$$

so that $P \iota\left(e_{\alpha}\right)=\iota\left(e_{\alpha}\right)$. Similarly, $\iota\left(e_{\alpha}\right) P=\iota\left(e_{\alpha}\right)$ for every $\alpha$. Hence

$$
\left\langle\mu, P^{2}\right\rangle=\lim _{\alpha}\left\langle\mu \cdot P, \iota\left(e_{\alpha}\right)\right\rangle=\lim _{\alpha}\left\langle\mu, P \iota\left(e_{\alpha}\right)\right\rangle=\lim _{\alpha}\left\langle\mu, \iota\left(e_{\alpha}\right)\right\rangle=\langle\mu, P\rangle
$$

for $\mu \in X$, hence $P$ is a projection. As $\left(P \iota\left(e_{\alpha}\right)\right)=\left(\iota\left(e_{\alpha}\right)\right)$ is a bounded approximate identity for $\mathcal{A}(E) \subseteq \mathcal{B}(E)$, the image of $P$ must be the whole of $E$, that is, $P$ is the identity. Thus

$$
\langle\mu, T\rangle=\langle\mu, P T\rangle=\left\langle T, \iota^{\prime}(\mu)\right\rangle=\left\langle\kappa_{E^{\prime} \widehat{\otimes} E^{\iota^{\prime}}}(\mu), T\right\rangle \quad(T \in \mathcal{B}(E), \mu \in X),
$$

and hence $\kappa_{E^{\prime} \widehat{\otimes} E}{ }^{\prime}$ is the identity on $X$. This implies that $X \subseteq \kappa_{E^{\prime} \widehat{\otimes} E}\left(E^{\prime} \widehat{\otimes} E\right)$, so that $X=\kappa_{E^{\prime} \widehat{\otimes} E}\left(E^{\prime} \widehat{\otimes} E\right)$, as required.

It would be nice to remove the condition on $E$ having the approximation property, but this is utterly integral to the current proof. The general question of which dual Banach algebras have a unique predual seems very interesting. We have looked at, but have been unable to answer, the question of whether $\ell^{1}(\mathbb{Z})$ has a unique predual. This is equivalent to the very concrete question: let $X \subseteq \ell^{\infty}(\mathbb{Z})$ be a shift-invariant subspace such that $X^{\prime}$ is naturally identified with $\ell^{1}(\mathbb{Z})$. Is $X=c_{0}(\mathbb{Z})$ ? Of course, as a Banach space, $\ell^{1}(\mathbb{Z})$ has plenty of preduals (see [15]) but these do not appear to be preduals which make the product on $\ell^{1}(\mathbb{Z})$ weak ${ }^{*}$-continuous.

5. Dual Banach $*$-algebras. We start by studying dual Banach algebras $\left(\mathcal{A}, \mathcal{A}_{*}\right)$ which admit an involution, which for us will be a continuous, conjugate-linear map $*: \mathcal{A} \rightarrow \mathcal{A}$ such that $(a b)^{*}=b^{*} a^{*}$. Recall that we may define an involution $*$ on $\mathcal{A}^{\prime}$ by

$$
\left\langle\mu^{*}, a\right\rangle=\overline{\left\langle\mu, a^{*}\right\rangle} \quad\left(\mu \in \mathcal{A}^{\prime}, a \in \mathcal{A}\right) .
$$

Then the involution is weak* ${ }^{*}$-continuous if and only if $\mathcal{A}_{*}$ forms a self-adjoint subspace of $\mathcal{A}^{\prime}$.

We shall now sketch some results on Banach spaces which admit a sesquilinear form which is not necessarily positive (such spaces are hence generalisations of Hilbert spaces). These are studied by Laustsen and the author in [12]. Let $E$ be a Banach space and let $[\cdot, \cdot]$ be a sesquilinear form on $E$ which is bounded in the sense that for some $C>0,|[x, y]| \leq C\|x\|\|y\|$ for $x, y \in E$. There hence exists a bounded, conjugate-linear map $J: E \rightarrow E^{\prime}$ 
such that $[x, y]=\langle J(y), x\rangle$ for any $x, y \in E$. Suppose that $J$ is a homeomorphism (which forces $E$ to be reflexive). Then there is an involution on $\mathcal{B}(E)$ given equivalently by

$$
T^{*}=J^{-1} \circ T^{\prime} \circ J \quad \text { or } \quad\left[T^{*}(x), y\right]=[x, T(y)] \quad(x, y \in E, T \in \mathcal{B}(E)) .
$$

It is shown in [12] that every bounded involution on $\mathcal{B}(E)$ arises in this way.

Now let $\left(\mathcal{A}, \mathcal{A}_{*}\right)$ be a dual Banach algebra with a continuous involution, let $\mu \in \mathcal{A}_{*}$ be self-adjoint and of norm one, and consider the space $E_{\mu}$ formed in Theorem 3.6. As this space is isomorphic to a Lions-Peetre interpolation space, it is isomorphic to the spaces constructed in [12]. In particular, we may define a bounded form on $E_{\mu}$ by

$$
[a \cdot \mu, b \cdot \mu]=\left\langle b^{*} a, \mu\right\rangle \quad(a, b \in \mathcal{A})
$$

such that the induced map $J: E \rightarrow E^{\prime}$ is a homeomorphism. It hence follows that the representation $\mathcal{A} \rightarrow \mathcal{B}(E)$ is actually a $*$-homomorphism.

The space $(E,[\cdot, \cdot])$ may certainly fail to be a Hilbert space. However, it is shown that in the special case when $\mu$ is a positive linear functional, $E$ is at least isomorphic to the Hilbert space generated by the GNS representation for $\mu$.

Proposition 5.1. Let $\left(\mathcal{A}, \mathcal{A}_{*}\right)$ be a dual Banach algebra with a continuous involution. Then the following are equivalent:

(1) the involution is weak ${ }^{*}$-continuous;

(2) $\mathcal{A}$ is weak ${ }^{*}$-continuously $*$-isomorphic to a closed subspace of $\mathcal{B}(E)$ for some reflexive Banach space $E$ such that $\mathcal{B}(E)$ admits an involution.

Proof. To show that (1) implies (2), by the preceding discussion, the only thing to check is that the subset of $\mathcal{A}_{*}$ of norm-one self-adjoint functionals norms $\mathcal{A}$. However, as the involution is weak*-continuous, $\mathcal{A}_{*}$ is itself selfadjoint, and hence every $\mu \in \mathcal{A}_{*}$ is of the form $\mu=\mu_{r}+i \mu_{i}$ for self-adjoint $\mu_{r}, \mu_{i} \in \mathcal{A}_{*}$. It hence follows that the quantity $\sup \left\{|\langle a, \mu\rangle|: \mu \in \mathcal{A}_{*}, \mu^{*}=\mu\right.$, $\|\mu\|=1\}$ is at least equivalent to $\|a\|$, as required.

Now suppose that (2) holds. We can then identify $\mathcal{A}$ with its image in $\mathcal{B}(E)$, so that $\mathcal{A}_{*}$ becomes identified with $E^{\prime} \widehat{\otimes} E /{ }^{\perp} \mathcal{A}_{*}$. Let $\tau \in \mathcal{A}_{*}$, so that as $J$ is a homeomorphism, $\tau$ has a representation of the form

$$
\tau=\sum_{n=1}^{\infty} J\left(x_{n}\right) \otimes y_{n}+{ }^{\perp} \mathcal{A}_{*},
$$

with $\sum_{n=1}^{\infty}\left\|x_{n}\right\|\left\|y_{n}\right\|<\infty$. Let

$$
\sigma=\sum_{n=1}^{\infty} J\left(y_{n}\right) \otimes x_{n}+{ }^{\perp} \mathcal{A}_{*} \in \mathcal{A}_{*} .
$$


Then, for $T \in \mathcal{A} \subseteq \mathcal{B}(E)$, we have

$$
\langle T, \tau\rangle=\sum_{n=1}^{\infty}\left\langle J\left(x_{n}\right), T\left(y_{n}\right)\right\rangle=\sum_{n=1}^{\infty}\left[T\left(y_{n}\right), x_{n}\right]=\sum_{n=1}^{\infty} \overline{\left[T^{*}\left(x_{n}\right), y_{n}\right]}=\overline{\left\langle T^{*}, \sigma\right\rangle}
$$

so that $\left\langle T, \sigma^{*}\right\rangle=\overline{\left\langle T^{*}, \sigma\right\rangle}=\langle T, \tau\rangle$. Therefore $\sigma^{*}=\tau$, so $\tau^{*}=\sigma$, and hence $\mathcal{A}_{*}$ is self-adjoint, as required.

It would be interesting to know if there exists a dual Banach algebra $\left(\mathcal{A}, \mathcal{A}_{*}\right)$ which admits a continuous involution which is not weak*-continuous.

Lemma 5.2. Let $\left(\mathcal{A}, \mathcal{A}_{*}\right)$ be a dual Banach algebra, and suppose that $\mathcal{A}$ admits a weak ${ }^{*}$-continuous involution. Then there is an equivalent norm $\|\cdot\|_{0}$ on $\mathcal{A}_{*}$ such that the involution becomes isometric on $\left(\mathcal{A},\|\cdot\|_{0}^{*}\right)$, where $\|\cdot\|_{0}^{*}$ is the dual norm to $\|\cdot\|_{0}$.

Proof. We would usually define a new norm on $\mathcal{A}$ by setting $\|a\| \|=$ $\max \left(\|a\|,\left\|a^{*}\right\|\right)$; we show here how to dualise this idea. For $\mu \in A_{*}$, we define

$$
\|\mu\|_{0}=\inf \left\{\left\|\lambda^{*}\right\|+\|\mu-\lambda\|: \lambda \in \mathcal{A}_{*}\right\},
$$

where, as $\mathcal{A}_{*}$ is self-adjoint, $\lambda^{*} \in \mathcal{A}_{*}$. Clearly, $\|\mu\| \leq\|\mu\|_{0}$ for $\mu \in \mathcal{A}_{*}$. As the involution is continuous, there exists $M \geq 1$ such that $\left\|a^{*}\right\| \leq M\|a\|$ for each $a \in \mathcal{A}$. Then $\left\|\lambda^{*}\right\| \leq M\|\lambda\|$ for each $\lambda \in \mathcal{A}^{\prime}$, so that also $\|\lambda\| \leq M\left\|\lambda^{*}\right\|$. Thus

$$
\|\mu\| \leq\|\lambda\|+\|\mu-\lambda\| \leq M\left\|\lambda^{*}\right\|+\|\mu-\lambda\| \leq M\left\|\lambda^{*}\right\|+M\|\mu-\lambda\| \quad\left(\lambda \in \mathcal{A}_{*}\right)
$$

so that $\|\mu\| \leq M\|\mu\|_{0}$. Hence $\|\cdot\|_{0}$ is equivalent to $\|\cdot\|$ on $\mathcal{A}_{*}$.

Then, for $a \in \mathcal{A}$, we have

$$
\begin{aligned}
\|a\|_{0}^{*} & =\sup \left\{|\langle a, \mu+\lambda\rangle|: \mu, \lambda \in \mathcal{A}_{*},\left\|\lambda^{*}\right\|+\|\mu\| \leq 1\right\} \\
& =\sup \left\{|\langle a, \mu\rangle|+|\langle a, \lambda\rangle|: \mu, \lambda \in \mathcal{A}_{*},\left\|\lambda^{*}\right\|+\|\mu\| \leq 1\right\} \\
& =\max \left\{\sup \{|\langle a, \mu\rangle|:\|\mu\| \leq 1\}, \sup \left\{|\langle a, \lambda\rangle|:\left\|\lambda^{*}\right\| \leq 1\right\}\right\} \\
& =\max \left\{\|a\|, \sup \left\{\left|\left\langle a^{*}, \lambda\right\rangle\right|:\|\lambda\| \leq 1\right\}\right\}=\max \left(\|a\|,\left\|a^{*}\right\|\right) .
\end{aligned}
$$

Hence $\left\|a^{*}\right\|_{0}^{*}=\|a\|_{0}^{*}$, as required.

Following [8], we shall say that a Banach *-algebra is a Banach algebra with an isometric involution. We now know that there is no loss of generality to talk about dual Banach *-algebras as long as the involution is weak*-continuous, which in light of Proposition 5.1 seems necessary for our purposes. The next theorem shows that we can always embed a dual Banach algebra with involution into a dual Banach algebra with weak*-continuous involution. We remind the reader of Proposition 2.4.

THEOREM 5.3. Let $\left(\mathcal{A}, \mathcal{A}_{*}\right)$ be a dual Banach algebra with a continuous involution. Then $\operatorname{WAP}\left(\mathcal{A}^{\prime}\right)^{\prime}$ admits a weak* ${ }^{*}$-continuous involution such that the canonical map $\mathcal{A} \rightarrow \operatorname{WAP}\left(\mathcal{A}^{\prime}\right)^{\prime}$ becomes a *-homomorphism. 
Proof. By Proposition 2.4, the first Arens product on $\mathcal{A}^{\prime \prime}$ drops to a welldefined product on $\operatorname{WAP}\left(\mathcal{A}^{\prime}\right)^{\prime}$ turning $\mathrm{WAP}\left(\mathcal{A}^{\prime}\right)^{\prime}$ into a dual Banach algebra. As shown in [12], it follows from Grothendieck's double limit criterion that $\operatorname{WAP}\left(\mathcal{A}^{\prime}\right)$ is a self-adjoint subspace of $\mathcal{A}^{\prime}$; in particular, this means that for $\mu \in \mathcal{A}_{*}$, certainly $\mu^{*} \in \operatorname{WAP}\left(\mathcal{A}^{\prime}\right)$.

We define an involution on $\mathcal{A}^{\prime \prime}$ by setting

$$
\left\langle\Phi^{*}, \lambda\right\rangle=\overline{\left\langle\Phi, \lambda^{*}\right\rangle} \quad\left(\Phi \in \mathcal{A}^{\prime \prime}, \lambda \in \mathcal{A}^{\prime}\right) .
$$

We then define an involution on $\operatorname{WAP}\left(\mathcal{A}^{\prime}\right)^{\prime}$ by setting

$$
\left(\Phi+\operatorname{WAP}\left(\mathcal{A}^{\prime}\right)^{\perp}\right)^{*}=\Phi^{*}+\operatorname{WAP}\left(\mathcal{A}^{\prime}\right)^{\perp} \quad\left(\Phi \in \mathcal{A}^{\prime \prime}\right),
$$

which is well-defined, as $\operatorname{WAP}\left(\mathcal{A}^{\prime}\right)$ is self-adjoint. We may check that, for $a \in \mathcal{A}, \lambda \in \operatorname{WAP}\left(\mathcal{A}^{\prime}\right)$ and $\Phi \in \mathcal{A}^{\prime \prime}$, we have $(a \cdot \lambda)^{*}=\lambda^{*} \cdot a^{*}$ and $(\lambda \cdot \Phi)^{*}=\Phi^{*} \cdot \lambda$. We then see that for $\Phi, \Psi \in \mathcal{A}^{\prime \prime}$ and $\lambda \in \operatorname{WAP}\left(\mathcal{A}^{\prime}\right)$,

$$
\begin{aligned}
\left\langle(\Phi \square \Psi)^{*}, \lambda\right\rangle & =\overline{\left\langle\Phi, \Psi \cdot \lambda^{*}\right\rangle}=\left\langle\Phi^{*},\left(\Psi \cdot \lambda^{*}\right)^{*}\right\rangle=\left\langle\Phi^{*}, \lambda \cdot \Psi^{*}\right\rangle \\
& =\left\langle\Psi^{*} \diamond \Phi^{*}, \lambda\right\rangle=\left\langle\Psi^{*} \square \Phi^{*}, \lambda\right\rangle,
\end{aligned}
$$

by Lemma 2.3. Thus $\operatorname{WAP}\left(\mathcal{A}^{\prime}\right)^{\prime}$ has a continuous involution which, by definition, is weak*-continuous, and extends the involution on $\mathcal{A}$.

Instead of using $\operatorname{WAP}\left(\mathcal{A}^{\prime}\right)$ in the above construction, we could instead have used $X=\mathcal{A}_{*}+\mathcal{A}_{*}^{*} \subseteq \operatorname{WAP}\left(\mathcal{A}^{\prime}\right)$, which has the advantage that if the involution on $\mathcal{A}$ is already weak ${ }^{*}$-continuous, then $X^{\prime}=\mathcal{A}$.

6. Connes-amenability and injectivity. We shall show below that if $\mathcal{A}$ is Connes-amenable, then $\mathcal{A}$ is unital. In fact, a stronger result holds.

Proposition 6.1. Let $\left(\mathcal{A}, \mathcal{A}_{*}\right)$ be a dual Banach algebra such that $\left(\mathcal{A}^{b}, \mathcal{A}_{*}^{b}\right)$ is Connes-amenable. Then $\mathcal{A}$ is unital.

Proof. Let $E=\mathcal{A}_{*}$ as a Banach space, and for $\mu \in \mathcal{A}_{*}$, write $\widehat{\mu}$ for the canonical image of $\mu$ in $E$ (and similarly for elements of $\mathcal{A}$ in $E^{\prime}$ ). Then turn $E$ into an $\mathcal{A}^{b}$-bimodule by setting

$$
(a+\alpha) \cdot \widehat{\mu}=\widehat{a \cdot \mu}+\alpha \widehat{\mu}, \quad \widehat{\mu} \cdot(a+\alpha)=\alpha \widehat{\mu} \quad\left(a+\alpha \in \mathcal{A}^{b}, \widehat{\mu} \in E\right) .
$$

We claim that $E^{\prime}$ then becomes a normal $\mathcal{A}^{b}$-bimodule; for example, if $a_{i}+\alpha_{i} \rightarrow a+\alpha$ weak $^{*}$ in $\mathcal{A}^{b}$ then, for $\widehat{\mu} \in E$ and $\widehat{b} \in E^{\prime}$,

$$
\begin{aligned}
\lim _{i}\left\langle\widehat{b} \cdot\left(a_{i}+\alpha_{i}\right), \widehat{\mu}\right\rangle & =\lim _{i}\left\langle\widehat{b}, \widehat{a_{i} \cdot \mu}+\alpha_{i} \widehat{\mu}\right\rangle=\lim _{i}\left\langle b a_{i}+\alpha_{i} b, \mu\right\rangle \\
& =\langle b a+\alpha b, \mu\rangle=\langle\widehat{b} \cdot(a+\alpha), \widehat{\mu}\rangle,
\end{aligned}
$$

so that $\widehat{b} \cdot\left(a_{i}+\alpha_{i}\right) \rightarrow \widehat{b} \cdot(a+\alpha)$ weak $^{*}$ in $E^{\prime}$, as required.

Now let $d: \mathcal{A}^{b} \rightarrow E^{\prime}$ be defined by $d(a+\alpha)=\widehat{a}$. Then

$$
d((a+\alpha)(b+\beta))=\widehat{a b}+\alpha \widehat{b}+\beta \widehat{a}=\widehat{a} \cdot(b+\beta)+(a+\alpha) \cdot \widehat{b},
$$


so that $d$ is a derivation. Clearly $d$ is weak ${ }^{*}$-continuous, hence as $\left(\mathcal{A}^{b}, \mathcal{A}_{*}^{b}\right)$ is Connes-amenable, $d$ is inner, so that for some $e \in \mathcal{A}$, we have

$$
\widehat{a}=d(a+\alpha)=\widehat{e} \cdot(a+\alpha)-(a+\alpha) \cdot \widehat{e}=\widehat{e a} \quad(a \in \mathcal{A}) .
$$

Thus $\mathcal{A}$ has a left identity, and in an analogous manner, $\mathcal{A}$ has a right identity, so that $\mathcal{A}$ is unital.

Thus the naive unitisation is useless as far as Connes-amenability is concerned.

Let $E$ be a Banach space, and let $\mathcal{A} \subseteq \mathcal{B}(E)$ be a subset. We define the commutant of $\mathcal{A}$ to be

$$
\mathcal{A}^{\mathrm{c}}=\{T \in \mathcal{B}(E): T S=S T(S \in \mathcal{A})\},
$$

so that $\mathcal{A}^{\mathrm{c}}$ is a closed subalgebra of $\mathcal{B}(E)$. We then define $\mathcal{A}^{\mathrm{cc}}=\left(\mathcal{A}^{\mathrm{c}}\right)^{\mathrm{c}}$, and see that $\mathcal{A} \subseteq \mathcal{A}^{\mathrm{cc}}$.

Definition 6.2. Let $E$ be a Banach space, and let $\mathcal{A} \subseteq \mathcal{B}(E)$ be a subalgebra. A quasi-expectation for $\mathcal{A}$ is a projection $\mathcal{Q}: \mathcal{B}(E) \rightarrow \mathcal{A}^{\mathrm{c}}$ such that $\mathcal{Q}(c T d)=c \mathcal{Q}(T) d$ for $c, d \in \mathcal{A}^{\mathrm{c}}$ and $T \in \mathcal{B}(E)$.

Proposition 6.3. Let $\mathcal{A}$ be a Banach algebra, and let $\pi: \mathcal{A} \rightarrow \mathcal{B}(E)$ be a homomorphism with $E$ a reflexive Banach space. Suppose that either

(1) $\mathcal{A}$ is amenable, or

(2) $\mathcal{A}$ is a dual Banach algebra, $\pi$ is weak ${ }^{*}$-continuous, and $\mathcal{A}$ is Connesamenable.

Then there exists a quasi-expectation $\mathcal{Q}: \mathcal{B}(E) \rightarrow \pi(\mathcal{A})^{\mathrm{c}}$.

Proof. We may either translate, almost verbatim, the proof of [5, Theorem 3], or else look at [29, Theorem 4.4.11].

Very similar ideas to the above are considered by Corach and Gale in [7], and in particular in Section 3 of that paper, where they ask if the existence of a quasi-expectation is equivalent to some form of amenability. We shall now answer this question in the affirmative, showing that quasi-expectations and Connes-amenability are intimately linked (as is true in the von Neumann algebra case: see [5]).

Definition 6.4. Let $\left(\mathcal{A}, \mathcal{A}_{*}\right)$ be a dual Banach algebra, and let $E$ be a Banach $\mathcal{A}$-bimodule. Then $x \in \sigma \mathrm{WC}(E)$ if and only if the maps $\mathcal{A} \rightarrow E$,

$$
a \mapsto a \cdot x, \quad a \mapsto x \cdot a,
$$

are $\sigma\left(\mathcal{A}, \mathcal{A}_{*}\right)-\sigma\left(E, E^{\prime}\right)$ continuous.

It is clear that $\sigma \mathrm{WC}(E)$ is a closed submodule of $E$. The $\mathcal{A}$-bimodule homomorphism $\Delta_{\mathcal{A}}$ has adjoint $\Delta_{\mathcal{A}}^{\prime}: \mathcal{A}^{\prime} \rightarrow(\mathcal{A} \widehat{\otimes} \mathcal{A})^{\prime}$. In [28, Corollary 4.6] it is shown that $\Delta_{\mathcal{A}}^{\prime}\left(\mathcal{A}_{*}\right) \subseteq \sigma \mathrm{WC}\left((\mathcal{A} \widehat{\otimes} \mathcal{A})^{\prime}\right)$. Consequently, we can view $\Delta_{\mathcal{A}}^{\prime}$ 
as a map $\mathcal{A}_{*} \rightarrow \sigma \mathrm{WC}\left((\mathcal{A} \widehat{\otimes} \mathcal{A})^{\prime}\right)$, denoted by $\widetilde{\Delta}_{\mathcal{A}}$, and hence we have a map $\widetilde{\Delta}_{\mathcal{A}}^{\prime}: \sigma \mathrm{WC}\left((\mathcal{A} \widehat{\otimes} \mathcal{A})^{\prime}\right)^{\prime} \rightarrow \mathcal{A}_{*}^{\prime}=\mathcal{A}$. The following is [28, Theorem 4.8].

TheOREM 6.5. Let $\mathcal{A}$ be a dual Banach algebra with predual $\mathcal{A}_{*}$. Then the following are equivalent:

(1) $\mathcal{A}$ is Connes-amenable;

(2) $\mathcal{A}$ has a $\sigma \mathrm{WC}$-virtual diagonal, which is $M \in \sigma \mathrm{WC}\left((\mathcal{A} \widehat{\otimes} \mathcal{A})^{\prime}\right)^{\prime}$ such that $a \cdot M=M \cdot a$ and $a \widetilde{\Delta}_{\mathcal{A}}^{\prime}(M)=a$ for each $a \in \mathcal{A}$.

We can identify $\sigma \mathrm{WC}\left((\mathcal{A} \widehat{\otimes} \mathcal{A})^{\prime}\right)^{\prime}$ in a slightly more concrete way. First of all, recall that $(\mathcal{A} \widehat{\otimes} \mathcal{A})^{\prime}=\mathcal{B}\left(\mathcal{A}, \mathcal{A}^{\prime}\right)$, where we choose the convention that

$$
\langle T, a \otimes b\rangle=\langle T(b), a\rangle \quad\left(a \otimes b \in \mathcal{A} \widehat{\otimes} \mathcal{A}, T \in \mathcal{B}\left(\mathcal{A}, \mathcal{A}^{\prime}\right)\right) .
$$

Then, for $\mu \in \mathcal{A}_{*}$, we identify $\widetilde{\Delta}_{\mathcal{A}}(\mu)$ with the map $b \mapsto b \cdot \mu$. The following is [11, Proposition 3.2].

Proposition 6.6. Let $\left(\mathcal{A}, \mathcal{A}_{*}\right)$ be a dual Banach algebra. For $T \in$ $\mathcal{B}\left(\mathcal{A}, \mathcal{A}^{\prime}\right)=(\mathcal{A} \widehat{\otimes} \mathcal{A})^{\prime}$, define maps $\phi_{r}, \phi_{l}: \mathcal{A} \widehat{\otimes} \mathcal{A} \rightarrow \mathcal{A}^{\prime}$ by

$$
\phi_{r}(a \otimes b)=T^{\prime} \kappa_{\mathcal{A}}(a) \cdot b, \quad \phi_{l}(a \otimes b)=a \cdot T(b) \quad(a \otimes b \in \mathcal{A} \widehat{\otimes} \mathcal{A}) .
$$

Then $T \in \sigma \mathrm{WC}\left(\mathcal{B}\left(\mathcal{A}, \mathcal{A}^{\prime}\right)\right)$ if and only if $\phi_{r}$ and $\phi_{l}$ are weakly compact and have ranges contained in $\kappa_{\mathcal{A}_{*}}\left(\mathcal{A}_{*}\right)$.

As the unit ball of $\mathcal{A} \widehat{\otimes} \mathcal{A}$ is the closure of the convex hull of $\{a \otimes b:\|a\|=$ $\|b\| \leq 1\}$, we see that, for example, $\phi_{l}$ is weakly compact if and only if the set $\{a \cdot T(b):\|a\|=\|b\| \leq 1\}$ is relatively weakly (sequentially) compact.

We shall now prove a representation result for maps in $\sigma \mathrm{WC}\left(\mathcal{B}\left(\mathcal{A}, \mathcal{A}^{\prime}\right)\right)$. Firstly, we again apply some interpolation space theory.

Proposition 6.7. Let $\left(\mathcal{A}, \mathcal{A}_{*}\right)$ be a unital dual Banach algebra, and let $T \in \sigma \mathrm{WC}\left(\mathcal{B}\left(\mathcal{A}, \mathcal{A}^{\prime}\right)\right)$. Then there exists an absolute constant $K>0$, a $B a$ nach left $\mathcal{A}$-module $E$ and a Banach right $\mathcal{A}$-module $F$ such that $E$ and $F$ are normal and reflexive, and for some unit vectors $\mu_{0} \in E^{\prime}$ and $\lambda_{0} \in F^{\prime}$, we have

$$
|\langle T(b), a\rangle| \leq K\|T\|\left\|\mu_{0} \cdot a\right\|_{E^{\prime}}\left\|b \cdot \lambda_{0}\right\|_{F^{\prime}} \quad(a, b \in \mathcal{A}) .
$$

Furthermore, $\mu_{0} \cdot \mathcal{A}$ is dense in $E^{\prime}$ and $\mathcal{A} \cdot \lambda_{0}$ is dense in $F^{\prime}$.

Proof. We may suppose that $\|T\|=1$. Form $\phi_{l}$ using $T$, and for $n \geq 1$ define a norm on $\mathcal{A}_{*}$ by

$$
\|\mu\|_{n}=\inf \left\{2^{-n / 2}\|\tau\|+2^{n / 2}\left\|\mu-\phi_{l}(\tau)\right\|: \tau \in \mathcal{A} \widehat{\otimes} \mathcal{A}\right\} \quad\left(\mu \in \mathcal{A}_{*}\right) .
$$

As in the proof of Theorem 3.6, we may check that $\|\cdot\|_{n}$ is an equivalent norm on $\mathcal{A}_{*}$, and that we may define

$$
E=\left\{\mu \in \mathcal{A}_{*}:\|\mu\|_{E}:=\left(\sum_{n \geq 1}\|\mu\|_{n}^{2}\right)^{1 / 2}<\infty\right\} .
$$


Then $E$ is a Banach space, $\phi_{l}(\mathcal{A} \widehat{\otimes} \mathcal{A})$ is dense in $E$, and for $\tau \in \mathcal{A} \widehat{\otimes} \mathcal{A}$, we have $\left\|\phi_{l}(\tau)\right\| \leq\left\|\phi_{l}(\tau)\right\|_{E} \leq\|\tau\|_{\pi}$. Furthermore, $E$ is a Banach left $\mathcal{A}$-module as $\phi_{l}$ is a left $\mathcal{A}$-module homomorphism. Again, it follows from general interpolation space results that $E$ is reflexive, as $\phi_{l}$ is weakly compact.

Again, let $\iota_{E}: E \rightarrow \mathcal{A}_{*}$ be the inclusion map, so that $\iota_{E}^{\prime}: \mathcal{A} \rightarrow E^{\prime}$ has dense range. Let $\mu \in E, \iota_{E}^{\prime}(a) \in E^{\prime}$, and let $\left(b_{\alpha}\right)$ be a net in $\mathcal{A}$ which converges weak* to $b$. Then

$$
\left\langle\iota_{E}^{\prime}(a), b_{\alpha} \cdot \mu\right\rangle=\left\langle a b_{\alpha}, \mu\right\rangle \rightarrow\langle a b, \mu\rangle=\left\langle\iota_{E}^{\prime}(a), b \cdot \mu\right\rangle,
$$

so we see that the map $\mathcal{A} \rightarrow E, b \mapsto b \cdot \mu$, is weak ${ }^{*}$-continuous, that is, $E$ is normal. For each $n \geq 1$, let $\|\cdot\|_{n}^{*}$ be the dual norm to $\|\cdot\|_{n}$, defined on $\mathcal{A}$. For $a \in \mathcal{A}$, we have

$$
\begin{aligned}
\|a\|_{n}^{*} & =\sup \left\{\left|\left\langle a, \mu+\phi_{l}(\tau)\right\rangle\right|: 2^{-n / 2}\|\tau\|+2^{n / 2}\|\mu\| \leq 1\right\} \\
& =\sup \left\{2^{-n / 2}|\langle a, \mu\rangle|+2^{n / 2}\left|\left\langle\phi_{l}^{\prime}(a), \tau\right\rangle\right|:\|\tau\|+\|\mu\| \leq 1\right\} \\
& =\max \left(2^{-n / 2}\|a\|, 2^{n / 2}\left\|\phi_{l}^{\prime}(a)\right\|\right) .
\end{aligned}
$$

As $E$ isometrically embeds into the $\ell^{2}$-direct sum of the spaces $\left(\mathcal{A}_{*},\|\cdot\|_{n}\right)_{n \geq 1}$, we see that $E^{\prime}$ is isometrically a quotient of the $\ell^{2}$-direct sum of the spaces $\left(\mathcal{A},\|\cdot\|_{n}^{*}\right)_{n \geq 1}$. We hence see that, if we drop the map $\iota_{E}^{\prime}$ and identify $\mathcal{A}$ with a dense subspace of $E^{\prime}$, then for $a \in \mathcal{A}$,

$$
\|a\|_{E^{\prime}}=\inf \left\{\left(\sum_{n \geq 1} \max \left(2^{-n / 2}\left\|a_{n}\right\|, 2^{n / 2}\left\|\phi_{l}^{\prime}\left(a_{n}\right)\right\|\right)^{2}\right)^{1 / 2}: a=\sum_{n \geq 1} a_{n}\right\} .
$$

From [3, Section 1.5, Proposition 1] it follows that there is an absolute constant $K>0$ such that if we define

$$
\|a\|_{1}=\inf \left\{\left(\sum_{n \geq 1}\left(2^{-n / 2}\left\|a-a_{n}\right\|+2^{n / 2}\left\|\phi_{l}^{\prime}\left(a_{n}\right)\right\|\right)^{2}\right)^{1 / 2}:\left(a_{n}\right) \subseteq \mathcal{A}\right\},
$$

then $K^{-1}\|\cdot\|_{1} \leq\|\cdot\|_{E^{\prime}} \leq K\|\cdot\|_{1}$.

We analogously use $\phi_{r}$ to form a reflexive, normal, Banach right $\mathcal{A}$ module $F$, and we find a norm $\|\cdot\|_{2}$ on $\mathcal{A}$ such that $K^{-1}\|\cdot\|_{2} \leq\|\cdot\|_{F^{\prime}} \leq K\|\cdot\|_{2}$. Notice that for $a, b \in \mathcal{A},\langle T(b), a\rangle=\left\langle\phi_{l}^{\prime}(a)(b), e_{\mathcal{A}}\right\rangle$ and

$$
\begin{aligned}
\left\|\phi_{l}^{\prime}(a)(b)\right\| & =\sup \{|\langle T(b), a c\rangle|:\|c\| \leq 1\}=\sup \left\{\left|\left\langle\phi_{r}^{\prime}(b)\left(e_{\mathcal{A}}\right), a c\right\rangle\right|:\|c\| \leq 1\right\} \\
& \leq\left\|\phi_{r}^{\prime}(b)\right\|\|a\| .
\end{aligned}
$$

For $a, b \in \mathcal{A}$ and $\varepsilon>0$, choose $\left(a_{n}\right),\left(b_{n}\right) \subseteq \mathcal{A}$ such that $a=\sum_{n \geq 1} a_{n}$ and

$$
\begin{aligned}
& \left(\sum_{n \geq 1} \max \left(2^{-n / 2}\left\|a_{n}\right\|, 2^{n / 2}\left\|\phi_{l}^{\prime}\left(a_{n}\right)\right\|\right)^{2}\right)^{1 / 2} \leq\|a\|_{E^{\prime}}+\varepsilon, \\
& \left(\sum_{n \geq 1}\left(2^{-n / 2}\left\|b-b_{n}\right\|+2^{n / 2}\left\|\phi_{r}^{\prime}\left(b_{n}\right)\right\|\right)^{2}\right)^{1 / 2} \leq\|b\|_{2}+\varepsilon .
\end{aligned}
$$


We then use the Cauchy-Schwarz inequality to see that

$$
\begin{aligned}
|\langle T(b), a\rangle| & \leq\left\|\phi_{l}^{\prime}(a)(b)\right\| \leq \sum_{n \geq 1}\left\|\phi_{l}^{\prime}\left(a_{n}\right)(b)\right\| \\
& \leq \sum_{n \geq 1}\left\|\phi_{l}^{\prime}\left(a_{n}\right)\left(b_{n}-b\right)\right\|+\left\|\phi_{l}^{\prime}\left(a_{n}\right)\left(b_{n}\right)\right\| \\
& \leq \sum_{n \geq 1} 2^{n / 2}\left\|\phi_{l}^{\prime}\left(a_{n}\right)\right\| 2^{-n / 2}\left\|b_{n}-b\right\|+\sum_{n \geq 1} 2^{n / 2}\left\|\phi_{r}^{\prime}\left(b_{n}\right)\right\| 2^{-n / 2}\left\|a_{n}\right\| \\
& \leq 2\left(\|a\|_{E^{\prime}}+\varepsilon\right)\left(\|b\|_{2}+\varepsilon\right) .
\end{aligned}
$$

As $\varepsilon>0$ was arbitrary, we see that $|\langle T(b), a\rangle| \leq 2 K\|a\|_{E^{\prime}}\|b\|_{F^{\prime}}$. We may hence set $\mu_{0}=\iota_{E}^{\prime}\left(e_{\mathcal{A}}\right)$ and $\lambda_{0}=\iota_{F}^{\prime}\left(e_{\mathcal{A}}\right)$, so that as $\iota_{E}^{\prime}$ has dense range, $\mu_{0} \cdot \mathcal{A}$ is dense in $E^{\prime}$, and similarly for $\mathcal{A} \cdot \lambda_{0}$. It remains to check that these are unit vectors. However, as $\iota^{\prime}$ is norm-decreasing, $\left\|\mu_{0}\right\|_{E^{\prime}} \leq 1$, while conversely

$$
\begin{aligned}
\left\|\iota_{E}^{\prime}\left(e_{\mathcal{A}}\right)\right\| & =\sup \left\{\left|\left\langle e_{\mathcal{A}}, \phi_{l}(\tau)\right\rangle\right|:\left\|\phi_{l}(\tau)\right\|_{E} \leq 1\right\} \\
& \geq \sup \left\{\left|\left\langle e_{\mathcal{A}}, \phi_{l}(a \otimes b)\right\rangle\right|:\|a \otimes b\|_{\pi}=\|a\|\|b\| \leq 1\right\} \\
& =\sup \{|\langle a, T(b)\rangle|:\|a \mid=\| b \|=1\}=\|T\|=1 .
\end{aligned}
$$

Similarly, $\left\|\lambda_{0}\right\|_{F^{\prime}}=1$, and the proof is complete.

Notice that if $T=\widetilde{\Delta}_{\mathcal{A}}(\mu)$ for some $\mu \in \mathcal{A}_{*}$, then $\phi_{l}(a \otimes b)=a \cdot(b \cdot \mu)=$ $a b \cdot \mu$. It hence follows that the space $E$ constructed in the above proof is equal to the space constructed in Theorem 3.6.

Theorem 6.8. Let $\left(\mathcal{A}, \mathcal{A}_{*}\right)$ be a unital dual Banach algebra, and let $T \in$ $\mathcal{B}\left(\mathcal{A}, \mathcal{A}^{\prime}\right)$. Then the following are equivalent:

(1) $T \in \sigma \mathrm{WC}\left(\mathcal{B}\left(\mathcal{A}, \mathcal{A}^{\prime}\right)\right)$;

(2) there exist a normal reflexive Banach left $\mathcal{A}$-module $E, x \in E, \mu \in E^{\prime}$ and $S \in \mathcal{B}(E)$ such that $\langle T(b), a\rangle=\langle\mu \cdot a, S(b \cdot x)\rangle$ for each $a, b \in \mathcal{A}$.

Furthermore, in this case, there is an absolute constant $K>0$ such that we may choose $E, x, \mu$ and $S$ with $\|x\|\|\mu\|\|S\| \leq K\|T\|$.

Proof. If (2) holds, then let $\pi: \mathcal{A} \rightarrow \mathcal{B}(E)$ be the associated representation, and let $\pi_{*}: E^{\prime} \widehat{\otimes} E \rightarrow \mathcal{A}^{\prime}$ be the restriction of $\pi^{\prime}$ to $E^{\prime} \widehat{\otimes} E$. As $E$ is normal, the representation is weak ${ }^{*}$-continuous, and so $\pi_{*}$ maps into $\mathcal{A}_{*}$, and $\pi_{*}^{\prime}=\pi$. For $a, b \in \mathcal{A}$, we have

$$
\begin{aligned}
\langle T(b), a\rangle & =\langle\mu, \pi(a) S \pi(b)(x)\rangle=\left\langle a, \pi_{*}(S \pi(b)(x) \otimes \mu)\right\rangle \\
& =\left\langle b, \pi_{*}\left(x \otimes S^{\prime} \pi(a)^{\prime}(\mu)\right)\right\rangle,
\end{aligned}
$$

so that $T(\mathcal{A}) \subseteq \mathcal{A}_{*}$ and $T^{\prime} \kappa_{\mathcal{A}}(\mathcal{A}) \subseteq \mathcal{A}_{*}$.

Let $\left(a_{n}\right)$ and $\left(b_{n}\right)$ be bounded sequences in $\mathcal{A}$, and let $x \in E$. As $E$ is reflexive, the unit ball of $E$ is weakly sequentially compact, and so, by 
moving to subsequences if necessary, we may suppose that for some $y \in E$,

$$
\left\langle\mu, \pi\left(a_{n}\right) S \pi\left(b_{n}\right)(x)\right\rangle \rightarrow\langle\mu, y\rangle \quad\left(\mu \in E^{\prime}\right) .
$$

Then, for $c \in \mathcal{A}$ and $\mu \in E^{\prime}$,

$$
\begin{aligned}
\lim _{n \rightarrow \infty}\left\langle a_{n} \cdot T\left(b_{n}\right), c\right\rangle=\lim _{n \rightarrow \infty}\left\langle\mu, \pi\left(c a_{n}\right) S \pi\left(b_{n}\right)(x)\right\rangle \\
\quad=\lim _{n \rightarrow \infty}\left\langle\pi(c)^{\prime}(\mu), \pi\left(a_{n}\right) S \pi\left(b_{n}\right)(x)\right\rangle=\left\langle\pi(c)^{\prime}(\mu), y\right\rangle=\left\langle c, \pi_{*}(y \otimes \mu)\right\rangle .
\end{aligned}
$$

Combining this with the comments after Proposition 6.6, we see that $\phi_{l}$ (as defined using $T$ ) is weakly compact. A similar calculation shows that $\phi_{r}$ is weakly compact, and so we conclude that $T \in \sigma \mathrm{WC}\left(\mathcal{B}\left(\mathcal{A}, \mathcal{A}^{\prime}\right)\right)$ as required.

Conversely, let $T \in \sigma \mathrm{WC}\left(\mathcal{B}\left(\mathcal{A}, \mathcal{A}^{\prime}\right)\right)$, and form the spaces $E$ and $F$ using Proposition 6.7. The subspace $\mu_{0} \cdot \mathcal{A}$ is dense in $E^{\prime}$ and $\mathcal{A} \cdot \lambda_{0}$ is dense in $F^{\prime}$. Define $R \in\left(E^{\prime} \widehat{\otimes} F^{\prime}\right)^{\prime}=\mathcal{B}\left(F^{\prime}, E\right)$ by

$$
\left\langle R, \mu_{0} \cdot a \otimes b \cdot \lambda_{0}\right\rangle=\langle T(b), a\rangle \quad(a, b \in \mathcal{A}),
$$

so that $\|R\| \leq K\|T\|$. Let $G=E \oplus_{2} F^{\prime}$ (that is, the norm on $G$ is $\|(e, f)\|=$ $\left(\|e\|^{2}+\|f\|^{2}\right)^{1 / 2}$ for $e \in E$ and $\left.f \in F\right)$ so that $G^{\prime}=E^{\prime} \oplus_{2} F$, and $G$ is a normal, reflexive, Banach left $\mathcal{A}$-module. Define $S \in \mathcal{B}(G)$ by

$$
S\left(z, b \cdot \lambda_{0}\right)=\left(R\left(b \cdot \lambda_{0}\right), 0\right) \quad\left(z \in E, b \cdot \lambda_{0} \in F^{\prime}\right),
$$

and let $x=\left(0, \lambda_{0}\right) \in G, \mu=\left(\mu_{0}, 0\right) \in G^{\prime}$. Then, for $a, b \in \mathcal{A}$,

$$
\langle\mu \cdot a, S(b \cdot x)\rangle=\left\langle\left(\mu_{0} \cdot a, 0\right), R\left(b \cdot \lambda_{0}, 0\right)\right\rangle=\left\langle R, \mu_{0} \cdot a \otimes b \cdot \lambda_{0}\right\rangle=\langle T(b), a\rangle,
$$

as required.

Let $\mathcal{A}$ be a Banach algebra and $E$ be a left $\mathcal{A}$-module. Then we write ${ }_{\mathcal{A}} \mathcal{B}(E)$ for the collection of left $\mathcal{A}$-module homomorphisms, that is, maps $T \in$ $\mathcal{B}(E)$ such that $T(a \cdot x)=a \cdot T(x)$ for $a \in \mathcal{A}$ and $x \in E$. Similarly, we define $\mathcal{B}_{\mathcal{A}}(E)$ and ${ }_{\mathcal{A}} \mathcal{B}_{\mathcal{A}}(E)$ to be the collection of right $\mathcal{A}$-module homomorphisms and $\mathcal{A}$-bimodule homomorphisms, respectively.

Suppose now that $\mathcal{A}$ is a closed subalgebra of $\mathcal{B}(E)$ for some reflexive Banach space $E$. Then $\mathcal{B}(E)$ becomes a Banach $\mathcal{A}$-bimodule and a Banach $\mathcal{A}^{\mathrm{c}}$-bimodule in the obvious way. We turn $\mathcal{A}^{\mathrm{c}} \mathcal{B}_{\mathcal{A}^{\mathrm{c}}}(\mathcal{B}(E))$ into a Banach $\mathcal{A}$ module by setting

$$
(a \cdot \mathcal{S})(T)=a \mathcal{S}(T), \quad(\mathcal{S} \cdot a)(T)=\mathcal{S}(T) a \quad(a \in \mathcal{A}, T \in \mathcal{B}(E))
$$

for $\mathcal{S} \in \mathcal{A}^{\mathrm{c}} \mathcal{B}_{\mathcal{A}^{\mathrm{c}}}(\mathcal{B}(E))$. Notice that $\mathcal{B}(\mathcal{B}(E))$ is a dual Banach space with predual $\mathcal{B}(E) \widehat{\otimes}\left(E \widehat{\otimes} E^{\prime}\right)$. Let $X \subseteq \mathcal{B}(E) \widehat{\otimes}\left(E \widehat{\otimes} E^{\prime}\right)$ be the closure of the linear span of

$$
\left\{\begin{array}{c}
c T \otimes x \otimes \mu-T \otimes x \otimes c^{\prime}(\mu), \\
T c \otimes x \otimes \mu-T \otimes c(x) \otimes \mu
\end{array}: c \in \mathcal{A}^{\mathrm{c}}, T \in \mathcal{B}(E), x \in E, \mu \in E^{\prime}\right\} .
$$

Then, for example, if $\mathcal{S} \in \mathcal{B}(\mathcal{B}(E))$ satisfies $\left\langle\mathcal{S}, c T \otimes x \otimes \mu-T \otimes x \otimes c^{\prime}(\mu)\right\rangle$ $=0$ for each $c \in \mathcal{A}^{\mathrm{c}}, T \in \mathcal{B}(E), x \in E$ and $\mu \in E^{\prime}$, then $\mathcal{S}(c T)=c \mathcal{S}(T)$. We 
hence see that $X^{\perp}={ }_{\mathcal{A}^{c}} \mathcal{B}_{\mathcal{A}^{\mathrm{c}}}(\mathcal{B}(E))$, so that ${ }_{\mathcal{A}^{\mathrm{c}}} \mathcal{B}_{\mathcal{A}^{\mathrm{c}}}(\mathcal{B}(E))$ has the predual $\mathcal{B}(E) \widehat{\otimes} E \widehat{\otimes} E^{\prime} / X$.

Define $\theta: \mathcal{A} \widehat{\otimes} \mathcal{A} \rightarrow \mathcal{A}^{\mathrm{c}} \mathcal{B}_{\mathcal{A}^{\mathrm{c}}}(\mathcal{B}(E))$ by

$$
\theta(a \otimes b)(T)=a T b \quad(a, b \in \mathcal{A}, T \in \mathcal{B}(E)),
$$

so that $\theta$ is an $\mathcal{A}$-bimodule homomorphism. We then define $\psi: \mathcal{B}(E) \widehat{\otimes} E \widehat{\otimes}$ $E^{\prime} / X \rightarrow \mathcal{B}\left(\mathcal{A}, \mathcal{A}^{\prime}\right)$ by, for $a, b \in \mathcal{A}, x \in E, \mu \in E^{\prime}$ and $T \in \mathcal{B}(E)$,

$$
\langle\psi(T \otimes x \otimes \mu+X), a \otimes b\rangle=\langle\mu, \theta(a \otimes b)(T)(x)\rangle=\langle\mu, a T b(x)\rangle .
$$

A simple check shows that this is well-defined, and that $\|\psi\| \leq 1$. We turn $\mathcal{B}(E) \widehat{\otimes} E \widehat{\otimes} E^{\prime}$ into a Banach $\mathcal{A}$-bimodule by setting

$$
\begin{aligned}
a \cdot(T \otimes x \otimes \mu)=T \otimes a(x) \otimes \mu, & (T \otimes x \otimes \mu) \cdot a=T \otimes x \otimes a^{\prime}(\mu) \\
& \left(a \in \mathcal{A}, T \in \mathcal{B}(E), x \in E, \mu \in E^{\prime}\right) .
\end{aligned}
$$

Then $X$ is a sub- $\mathcal{A}$-bimodule, and this module action agrees with the module action already defined on $\mathcal{A}^{\mathrm{c}} \mathcal{B}_{\mathcal{A}^{\mathrm{c}}}(\mathcal{B}(E))$. We may verify that $\psi$ is an $\mathcal{A}$ bimodule homomorphism.

We now aim to construct a Banach space $E$ such that $\psi$ is a bijection (onto a suitable closed subspace of $\mathcal{B}\left(\mathcal{A}, \mathcal{A}^{\prime}\right)$ ) for this $E$.

Definition 6.9. For a Banach left $\mathcal{A}$-module $E$, and $x \in E$, let $\overline{\mathcal{A} \cdot x}$ be the closure of $\{a \cdot x: a \in \mathcal{A}\}$, so that $\overline{\mathcal{A} \cdot x}$ is a closed submodule of $E$. Similarly, for $\mu \in E^{\prime}$, define $\overline{\mu \cdot \mathcal{A}}$. We then say that $E$ is cyclic if, for some $x_{0} \in E$, we have $\overline{\mathcal{A} \cdot x_{0}}=E$; a similar definition holds for $E^{\prime}$.

For Banach spaces $E$ and $F$, we let $\ell^{2}(E \oplus F)=E \oplus_{2} F$ be the direct sum of $E$ and $F$ with the norm $\|(e, f)\|=\left(\|e\|^{2}+\|f\|^{2}\right)^{1 / 2}$ for $e \in E, f \in F$. When $E$ and $F$ are reflexive, normal, Banach left $\mathcal{A}$-modules, it is clear that so is $E \oplus_{2} F$. We similarly define $\ell^{2}\left(\bigoplus_{\alpha} E_{\alpha}\right)$, where $\left(E_{\alpha}\right)$ is a family of Banach spaces.

The following lemma is a technical result. It would be easier to define $E$ to be the $\ell^{2}$-direct-sum of all reflexive, normal, Banach left $\mathcal{A}$-modules, but this collection is not in general a set.

Lemma 6.10. Let $\mathcal{E}$ be a set of reflexive, normal, cyclic, Banach left $\mathcal{A}$-modules. There exists a reflexive, normal, Banach left $\mathcal{A}$-module $E$ such that:

(1) each member of $\mathcal{E}$ is isometrically isomorphic to a 1-complemented submodule of $E$;

(2) for $x_{1}, x_{2} \in E$ and $\mu_{1}, \mu_{2} \in E^{\prime}$, if $X\left(x_{1}, x_{2}\right)=\overline{\left\{a \cdot\left(x_{1}, x_{2}\right): a \in \mathcal{A}\right\}}$ $\subseteq \overline{\mathcal{A} \cdot x_{1}} \oplus_{2} \overline{\mathcal{A} \cdot x_{2}}$ and $Y\left(\mu_{1}, \mu_{2}\right)=\overline{\left\{\left(\mu_{1}, \mu_{2}\right) \cdot a: a \in \mathcal{A}\right\}} \subseteq \overline{\mu_{1} \cdot \mathcal{A}} \oplus_{2}$ $\overline{\mu_{2} \cdot \mathcal{A}}$, then $X\left(x_{1}, x_{2}\right) \oplus_{2} Y\left(\mu_{1}, \mu_{2}\right)^{\prime}$ is isometrically isomorphic to a 1-complemented submodule of $E$. 
Proof. Let $\mathcal{E}_{0}=\mathcal{E}$, and then use transfinite induction to define $\mathcal{E}_{\alpha}$, for an ordinal $\alpha$, as follows. If $\alpha$ is a limit ordinal, we let $\mathcal{E}_{\alpha}=\bigcup_{\lambda<\alpha} \mathcal{E}_{\lambda}$. Otherwise, let $E_{\alpha}=\ell^{2}\left(\bigoplus_{E \in \mathcal{E}_{\alpha}} E\right)$ and for $x_{1}, x_{2} \in E_{\alpha}$ and $\mu_{1}, \mu_{2} \in E_{\alpha}^{\prime}$, form $X\left(x_{1}, x_{2}\right)$ and $Y\left(\mu_{1}, \mu_{2}\right)$ as above. Then let $\mathcal{E}_{\alpha+1}$ be $\mathcal{E}_{\alpha}$ unioned with the collection of all such spaces $X\left(x_{1}, x_{2}\right) \oplus_{2} Y\left(\mu_{1}, \mu_{2}\right)^{\prime}$. Notice that each member of $\mathcal{E}_{\alpha}$ is canonically a reflexive, normal, Banach $\mathcal{A}$-module. Notice that $X\left(x_{1}, x_{2}\right)$ is always a cyclic module, while $Y\left(\mu_{1}, \mu_{2}\right)^{\prime}$ is the dual of a cyclic (right) module.

We then let $E=\ell^{2}\left(\bigoplus_{E \in \mathcal{E}_{\aleph_{1}}} E\right)$ and give $E$ the obvious left $\mathcal{A}$-module structure. Then each member of $\mathcal{E}$ is a 1-complemented subspace of $E$. Indeed, we may view $E_{\alpha}=\ell^{2}\left(\bigoplus_{E \in \mathcal{E}_{\alpha}} E\right)$ and $E_{\alpha}^{\prime}$ as submodules of $E$ and $E^{\prime}$, respectively, for each $\alpha<\aleph_{1}$. For notational convenience, let $\mathcal{E}_{\aleph_{1}}=\left\{E_{i}: i \in I\right\}$ for some indexing set $I$. Then let $x_{1}, x_{2} \in E$ and $\mu_{1}, \mu_{2} \in E^{\prime}$, so that, for $k=1,2, x_{k}=\left(x_{i}^{(k)}\right)_{i \in I}$ with $\left\|x_{k}\right\|=\left(\sum_{i \in I}\left\|x_{i}^{(k)}\right\|^{2}\right)^{1 / 2}$, and similarly for $\mu_{k}$. As $\aleph_{1}$ is the first uncountable ordinal, we see that for some $\alpha<\aleph_{1}$, for each $k=1,2$ the condition $x_{i}^{(k)} \neq 0$ or $\mu_{i}^{(k)} \neq 0$ implies that $E_{i} \in \mathcal{E}_{\alpha}$. Hence $x_{1}, x_{2} \in E_{\alpha}$ and $\mu_{1}, \mu_{2} \in E_{\alpha}^{\prime}$, so that by construction, $X\left(x_{1}, x_{2}\right) \oplus_{2} Y\left(\mu_{1}, \mu_{2}\right)^{\prime}$ is a 1-complemented submodule of $E_{\alpha+1} \subseteq E$ as required.

Theorem 6.11. Let $\left(\mathcal{A}, \mathcal{A}_{*}\right)$ be a unital dual Banach algebra. There exists an isometric, weak ${ }^{*}$-continuous representation $\pi: \mathcal{A} \rightarrow \mathcal{B}(E)$ such that $\psi$ (as associated with $\pi$ ) maps into $\sigma \mathrm{WC}\left(\mathcal{B}\left(\mathcal{A}, \mathcal{A}^{\prime}\right)\right.$ ) and is a bijection.

Proof. By Theorem 6.8, we see that $\psi$ maps into $\sigma \mathrm{WC}\left(\mathcal{B}\left(\mathcal{A}, \mathcal{A}^{\prime}\right)\right)$ for any isometric weak*-continuous representation $\pi: \mathcal{A} \rightarrow \mathcal{B}(E)$. Let $\mathcal{E}$ be the collection of Banach spaces constructed in Theorem 6.8 for each normone member of $\sigma \mathrm{WC}\left(\mathcal{B}\left(\mathcal{A}, \mathcal{A}^{\prime}\right)\right)$. Then let $E$ be the Banach space given by Lemma 6.10 , so that it is clear that $\psi$ is surjective, by Theorem 6.8. Hence $\psi^{\prime}$ is an isomorphism onto its range. We shall now show that $\psi^{\prime}$ is surjective, which will complete the proof.

Fix $\mathcal{S} \in{ }_{\mathcal{A}^{c}} \mathcal{B}_{\mathcal{A}^{c}}(\mathcal{B}(E))$, and define $M \in \sigma \mathrm{WC}\left(\mathcal{B}\left(\mathcal{A}, \mathcal{A}^{\prime}\right)\right)^{\prime}$ in the following way. For each $T \in \sigma \mathrm{WC}\left(\mathcal{B}\left(\mathcal{A}, \mathcal{A}^{\prime}\right)\right)$, let $x \in E, \mu \in E^{\prime}$ and $S \in \mathcal{B}(E)$ be such that $\langle T, a \otimes b\rangle=\langle\mu \cdot a, S(b \cdot x)\rangle$ for each $a, b \in \mathcal{A}$. Then define $\langle M, T\rangle=\langle\mu, \mathcal{S}(S)(x)\rangle$. Suppose that this is well-defined. Then, for each $x \in E, \mu \in E^{\prime}$ and $S \in \mathcal{B}(E)$,

$$
\begin{aligned}
\left\langle\psi^{\prime}(M), S \otimes x \otimes \mu+X\right\rangle & =\langle M, \psi(S \otimes x \otimes \mu+X)\rangle=\langle\mu, \mathcal{S}(S)(x)\rangle \\
& =\langle\mathcal{S}, S \otimes x \otimes \mu+X\rangle,
\end{aligned}
$$

so that $\psi^{\prime}(M)=\mathcal{S}$ as required.

We shall now show that $M$ is well-defined, at least for our specific $E$. Let $T \in \sigma \mathrm{WC}\left(\mathcal{B}\left(\mathcal{A}, \mathcal{A}^{\prime}\right)\right.$ ), and suppose that, for $i=1,2$, we have $x_{i} \in E$, $\mu_{i} \in E^{\prime}$ and $S_{i} \in \mathcal{B}(E)$ such that $\langle T, a \otimes b\rangle=\left\langle\mu_{i} \cdot a, S_{i}\left(b \cdot x_{i}\right)\right\rangle$ for $a, b \in \mathcal{A}$. Pick $t \in(0,1)$ such that $t\left\|S_{1}\right\|=(1-t)\left\|S_{2}\right\|$. A quick calculation shows that 
then $K:=t\left\|S_{1}\right\|=\left\|S_{1}\right\|\left\|S_{2}\right\|\left(\left\|S_{1}\right\|+\left\|S_{2}\right\|\right)^{-1}$. For each $a, b \in \mathcal{A}$, by the Cauchy-Schwarz inequality,

$$
\begin{aligned}
|\langle T, a \otimes b\rangle| & =t\left|\left\langle\mu_{1} \cdot a, S_{1}\left(b \cdot x_{1}\right)\right\rangle\right|+(1-t)\left|\left\langle\mu_{2} \cdot a, S_{2}\left(b \cdot x_{2}\right)\right\rangle\right| \\
& \leq t\left\|S_{1}\right\|\left\|\mu_{1} \cdot a\right\|\left\|b \cdot x_{1}\right\|+(1-t)\left\|S_{2}\right\|\left\|\mu_{2} \cdot a\right\|\left\|b \cdot x_{2}\right\| \\
& \leq K\left(\left\|\mu_{1} \cdot a\right\|^{2}+\left\|\mu_{2} \cdot a\right\|^{2}\right)^{1 / 2}\left(\left\|b \cdot x_{1}\right\|^{2}+\left\|b \cdot x_{2}\right\|^{2}\right)^{1 / 2}
\end{aligned}
$$

Let $F$ be the closure of $\mathcal{A} \cdot\left(x_{1}, x_{2}\right)$ in $\overline{\mathcal{A} \cdot x_{1}} \oplus_{2} \overline{\mathcal{A} \cdot x_{2}}$, and let $G$ be the closure of $\left(\mu_{1}, \mu_{2}\right) \cdot \mathcal{A}$ in $\overline{\mu_{1} \cdot \mathcal{A}} \oplus_{2} \overline{\mu_{2} \cdot \mathcal{A}}$. The above calculation allows us to define $R \in(G \widehat{\otimes} F)^{\prime}=\mathcal{B}\left(F, G^{\prime}\right)$ by

$$
\left\langle R,\left(\mu_{1} \cdot a, \mu_{2} \cdot a\right) \otimes\left(b \cdot x_{1}, b \cdot x_{2}\right)\right\rangle=\langle T, a \otimes b\rangle \quad(a, b \in \mathcal{A}),
$$

and we see that $\|R\| \leq K$. Then set $H=G^{\prime} \oplus_{2} F$, and let $P_{F}$ and $P_{G^{\prime}}$ be the projections onto $F$ and $G^{\prime}$ respectively. As $H^{\prime}=G \oplus_{2} F^{\prime}$, let $P_{G}$ and $P_{F^{\prime}}$ be defined similarly, so $P_{G}=P_{G^{\prime}}^{\prime}$ and $P_{F^{\prime}}=P_{F}^{\prime}$. Let $x_{0}=\left(0,\left(x_{1}, x_{2}\right)\right) \in H$, $\mu_{0}=\left(\left(\mu_{1}, \mu_{2}\right), 0\right) \in H^{\prime}$, and define $S_{0} \in \mathcal{B}(H)$ by $S_{0}\left(g^{*}, f\right)=(R(f), 0)$ for $g^{*} \in G^{\prime}$ and $f \in F$. Then, for $a, b \in \mathcal{A}$,

$$
\left\langle\mu_{0} \cdot a, S_{0}\left(b \cdot x_{0}\right)\right\rangle=\left\langle\left(\mu_{1} \cdot a, \mu_{2} \cdot a\right), R\left(b \cdot x_{1}, b \cdot x_{2}\right)\right\rangle=\langle T, a \otimes b\rangle .
$$

By Lemma 6.10, $H$ is a 1-complemented submodule of $E$, so we can find norm-decreasing left $\mathcal{A}$-module homomorphisms $P: E \rightarrow H$ and $\iota: H \rightarrow E$ such that $P \iota=I_{H}$. For $i=1,2$, we may define maps $U_{i} \in{ }_{\mathcal{A}} \mathcal{B}\left(H, \overline{\mathcal{A} \cdot x_{i}}\right)$ and $V_{i} \in \mathcal{B}_{\mathcal{A}}\left(H^{\prime}, \overline{\mu_{i} \cdot \mathcal{A}}\right)$ by, for $a \in \mathcal{A}, f^{*} \in F^{\prime}$ and $g^{*} \in G^{\prime}$,

$$
U_{i}\left(g^{*}, a \cdot\left(x_{1}, x_{2}\right)\right)=a \cdot x_{i}, \quad V_{i}\left(\left(\mu_{1}, \mu_{2}\right) \cdot a, f^{*}\right)=\mu_{i} \cdot a .
$$

Then, by construction, $U_{i}$ and $V_{i}$ are norm-decreasing. For $a, b \in \mathcal{A}$, we see that

$$
\begin{aligned}
\langle T, a \otimes b\rangle & =\left\langle\mu_{i} \cdot a, S_{i}\left(b \cdot x_{i}\right)\right\rangle=\left\langle\mu_{0} \cdot a, V_{i}^{\prime} S_{i} U_{i}\left(b \cdot x_{0}\right)\right\rangle \\
& =\left\langle\mu_{0} \cdot a, P_{G^{\prime}} V_{i}^{\prime} S_{i} U_{i} P_{F}\left(b \cdot x_{0}\right)\right\rangle=\left\langle\mu_{0} \cdot a, P_{G^{\prime}} S_{0} P_{F}\left(b \cdot x_{0}\right)\right\rangle .
\end{aligned}
$$

As $\mathcal{A} \cdot x_{0}$ is dense in $F$ and $\mu_{0} \cdot \mathcal{A}$ is dense in $G$, we conclude that $P_{G^{\prime}} V_{i}^{\prime} S_{i} U_{i} P_{F}$ $=P_{G^{\prime}} S_{0} P_{F}$. As $\mathcal{S} \in \mathcal{A}^{c} \mathcal{B}_{\mathcal{A}^{\mathrm{c}}}(\mathcal{B}(E))$, we see that

$$
\begin{aligned}
\left\langle\mu_{i}, \mathcal{S}\left(S_{i}\right)\left(x_{i}\right)\right\rangle & =\left\langle V_{i} P_{G}\left(\mu_{0}\right), \mathcal{S}\left(S_{i}\right) U_{i} P_{F}\left(x_{0}\right)\right\rangle \\
& =\left\langle\mu_{0}, P \iota P_{G^{\prime}} V_{i}^{\prime} \mathcal{S}\left(S_{i}\right) U_{i} P_{F} P \iota\left(x_{0}\right)\right\rangle \\
& =\left\langle\mu_{0}, P \mathcal{S}\left(\iota P_{G^{\prime}} V_{i}^{\prime} S_{i} U_{i} P_{F} P\right) \iota\left(x_{0}\right)\right\rangle \\
& =\left\langle\mu_{0}, P \mathcal{S}\left(\iota P_{G^{\prime}} S_{0} P_{F} P\right) \iota\left(x_{0}\right)\right\rangle .
\end{aligned}
$$

We hence conclude that $\left\langle\mu_{i}, \mathcal{S}\left(S_{i}\right)\left(x_{i}\right)\right\rangle$ has the same value for $i=1$ as for $i=2$, and hence that $M$ is well-defined, as required.

Definition 6.12. Let $\left(\mathcal{A}, \mathcal{A}_{*}\right)$ be a dual Banach algebra. We say that $\mathcal{A}$ is injective if whenever $\pi: \mathcal{A} \rightarrow \mathcal{B}(E)$ is a weak*-continuous, unital representation, there is a quasi-expectation $\mathcal{Q}: \mathcal{B}(E) \rightarrow \pi(\mathcal{A})^{\mathrm{c}}$. 
Theorem 6.13. Let $\left(\mathcal{A}, \mathcal{A}_{*}\right)$ be a unital dual Banach algebra. Then $\mathcal{A}$ is Connes-amenable if and only if $\mathcal{A}$ is injective.

Proof. We have already seen that when $\mathcal{A}$ is Connes-amenable, $\mathcal{A}$ is injective. Conversely, consider the weak ${ }^{*}$-continuous representation $\pi: \mathcal{A} \rightarrow$ $\mathcal{B}(E)$ constructed in Theorem 6.11 , so that $\psi^{\prime}$ is an isomorphism. As $\mathcal{A}$ is injective, there exists a quasi-expectation $\mathcal{Q}: \mathcal{B}(E) \rightarrow \pi(\mathcal{A})^{\mathrm{c}}$. Let $M=$ $\left(\psi^{\prime}\right)^{-1}(\mathcal{Q}) \in \sigma \mathrm{WC}\left((\mathcal{A} \widehat{\otimes} \mathcal{A})^{\prime}\right)^{\prime}$. As $\mathcal{Q}$ maps into $\pi(\mathcal{A})^{\mathrm{c}}$, it follows that $a \cdot \mathcal{Q}=$ $\mathcal{Q} \cdot a$ for $a \in \mathcal{A}$, so that $a \cdot M=M \cdot a$.

The unit ball of $\mathcal{A} \widehat{\otimes} \mathcal{A}$ is weak*-dense in the unit ball of $\sigma \mathrm{WC}\left((\mathcal{A} \widehat{\otimes} \mathcal{A})^{\prime}\right)^{\prime}$, so there exists a bounded net $\left(\tau_{\alpha}\right)$ in $\mathcal{A} \widehat{\otimes} \mathcal{A}$ such that $M$ is the weak*-limit of $\left(\tau_{\alpha}\right)$. For each $\alpha$, let $\tau_{\alpha}=\sum_{n>1} a_{n}^{(\alpha)} \otimes b_{n}^{(\alpha)}$. For $x \in E$ and $\mu \in E^{\prime}$, there exists $\lambda \in \mathcal{A}_{*}$ such that $\langle a, \lambda\rangle=\langle\mu, \pi(a)(x)\rangle$ for $a \in \mathcal{A}$. We then see that for $a, b \in \mathcal{A}$,

$$
\langle a b, \lambda\rangle=\left\langle\widetilde{\Delta}_{\mathcal{A}}(\lambda), a \otimes b\right\rangle=\langle\mu, \pi(a b)(x)\rangle=\left\langle\mu, \pi(a) I_{E} \pi(b)(\mu)\right\rangle .
$$

Then, from the proof of Theorem 6.11, we deduce that

$$
\left\langle\mu, \mathcal{Q}\left(I_{E}\right)(x)\right\rangle=\left\langle M, \widetilde{\Delta}_{\mathcal{A}}(\lambda)\right\rangle=\left\langle\widetilde{\Delta}_{\mathcal{A}}^{\prime}(M), \lambda\right\rangle=\lim _{\alpha} \sum_{n \geq 1}\left\langle\mu, \pi\left(a_{n}^{(\alpha)} b_{n}^{(\alpha)}\right)(x)\right\rangle .
$$

As $\mathcal{Q}$ is a projection onto $\pi(A)^{\mathrm{c}}$ and $I_{E} \in \pi(A)^{\mathrm{c}}$, we see $\mathcal{Q}\left(I_{E}\right)=I_{E}$, and so, as $x$ and $\mu$ were arbitrary, we must have $\lim _{\alpha} \sum_{n \geq 1} a_{n}^{(\alpha)} b_{n}^{(\alpha)}=e_{\mathcal{A}}$ in the weak*-topology on $\mathcal{A}$. That is, $\widetilde{\Delta}_{\mathcal{A}}^{\prime}(M)=e_{\mathcal{A}}$, showing that $M$ is a $\sigma \mathrm{WC}$ virtual diagonal, which implies that $\mathcal{A}$ is Connes-amenable, as required.

There exists a rather strong decomposition theory for weak*-continuous homomorphisms between von Neumann algebras (see [32, Theorem 5.5]). From this, it follows that if $\mathcal{A} \subseteq \mathcal{B}(H)$ is a von Neumann algebra admitting a quasi-expectation, and $\mathcal{A}$ is isomorphic to $\mathcal{B} \subseteq \mathcal{B}(K)$, then $\mathcal{B}$ admits a quasi-expectation (see [29, Lemma 6.1.2]). Hence we need only look at one representation for $\mathcal{A}$ to decide if $\mathcal{A}$ is Connes-amenable.

In contrast, it follows from [30, Corollary 4.5] that $\mathcal{A}=\mathcal{B}\left(\ell^{p} \oplus \ell^{q}\right)$ is not Connes-amenable when $p, q \in(1, \infty) \backslash\{2\}$ are distinct, while trivially, there is a quasi-expectation for $\mathcal{A}$ under the trivial representation to $\mathcal{B}\left(\ell^{p} \oplus \ell^{q}\right)$. Our theorem shows that there exists some reflexive Banach space $E$ and some weak*-continuous representation $\pi: \mathcal{A} \rightarrow \mathcal{B}(E)$ such that $\mathcal{A}$ has no quasi-expectation for $\mathcal{B}(E)$. It would be interesting to determine the Banach space properties of $E$.

There exists a more category-theoretic definition of injectivity for von Neumann algebras (see [33, Chapter XV, Section 1]): namely, they are injective in the usual mapping sense, with respect to completely positive maps. Does a similar definition hold for dual Banach algebras? 
Remark 6.14. Let $\left(\mathcal{A}, \mathcal{A}_{*}\right)$ be a dual Banach algebra, and let $\pi: \mathcal{A} \rightarrow$ $\mathcal{B}(E)$ be the representation given by Theorem 6.11. Then suppose that $\mathcal{B}=$ $\mathcal{A}^{\mathrm{cc}}$ is Connes-amenable, so that there exists a quasi-projection $\mathcal{Q}: \mathcal{B} \rightarrow$ $\left(\mathcal{A}^{\mathrm{cc}}\right)^{\mathrm{c}}=\mathcal{A}^{\mathrm{c}}$. Thus $\mathcal{A}^{\sharp}$ is Connes-amenable by Theorem 6.13 , which implies in particular that $\mathcal{A}$ is unital. Hence, if we wish to unitise $\mathcal{A}$ by using $\mathcal{A}^{\mathrm{cc}}$, then we need to consider "smaller" representations.

Proposition 6.15. Let $\mathcal{A}$ be a Banach algebra. Then the following are equivalent:

(1) $\operatorname{WAP}\left(\mathcal{A}^{\prime}\right)^{\prime}$ is Connes-amenable;

(2) whenever $\pi: \mathcal{A} \rightarrow \mathcal{B}(E)$ is a continuous representation on a reflexive Banach space $E$, there exists a quasi-expectation $\mathcal{Q}: \mathcal{B}(E) \rightarrow \pi(\mathcal{A})^{\mathrm{c}}$.

Proof. Let $\pi: \mathcal{A} \rightarrow \mathcal{B}(E)$ be a continuous representation on a reflexive Banach space $E$, so that by Proposition 2.9 , there is a unique weak*continuous representation $\widehat{\pi}: \operatorname{WAP}\left(\mathcal{A}^{\prime}\right)^{\prime} \rightarrow \mathcal{B}(E)$ extending $\pi$. It is hence sufficient to show that $\pi(\mathcal{A})^{\mathrm{c}}=\widehat{\pi}\left(\operatorname{WAP}\left(\mathcal{A}^{\prime}\right)^{\prime}\right)^{\mathrm{c}}$. The inclusion $\supseteq$ is clear. Conversely, let $T \in \pi(\mathcal{A})^{\mathrm{c}}$, so that

$$
\langle T \pi(a), \tau\rangle=\langle\pi(a) T, \tau\rangle \quad\left(a \in \mathcal{A}, \tau \in E^{\prime} \widehat{\otimes} E\right) .
$$

Then let $\Phi \in \operatorname{WAP}\left(\mathcal{A}^{\prime}\right)^{\prime}$ and let $\left(a_{\alpha}\right)$ be a bounded net in $\mathcal{A}$ which converges to $\Phi$ in the weak*-topology on $\operatorname{WAP}\left(\mathcal{A}^{\prime}\right)^{\prime}$. Consequently, for $x \in E, \mu \in E^{\prime}$ and $T \in \pi(\mathcal{A})^{\mathrm{c}}$,

$$
\begin{aligned}
\langle\mu, T \widehat{\pi}(\Phi)(x)\rangle & =\left\langle\Phi, \pi_{*}\left(T^{\prime}(\mu) \otimes x\right)\right\rangle=\lim _{\alpha}\left\langle\pi_{*}\left(T^{\prime}(\mu) \otimes x\right), a_{\alpha}\right\rangle \\
& =\lim _{\alpha}\left\langle\mu, T \pi\left(a_{\alpha}\right)(x)\right\rangle=\lim _{\alpha}\left\langle\mu, \pi\left(a_{\alpha}\right) T(x)\right\rangle \\
& =\lim _{\alpha}\left\langle\pi_{*}(\mu \otimes T(x)), a_{\alpha}\right\rangle=\left\langle\Phi, \pi_{*}(\mu \otimes T(x))\right\rangle \\
& =\langle\mu, \widehat{\pi}(\Phi) T(x)\rangle,
\end{aligned}
$$

so that $T \in \widehat{\pi}\left(\operatorname{WAP}\left(\mathcal{A}^{\prime}\right)^{\prime}\right)^{\mathrm{c}}$, as required.

7. WAP-compactifications for semigroups. Semigroup algebras fit very nicely into our framework, and the theory is well-explored. Here we shall sketch some results on compactifications; for further details, see [4].

Let $S$ be a semigroup which is also a topological space. Then $S$ is a semitopological semigroup when the left and right actions of $S$ are continuous, while $S$ is a topological semigroup when the multiplication map $S \times S \rightarrow S$ is continuous.

We write $\ell^{\infty}(S)$ for the commutative $\mathrm{C}^{*}$-algebra of all bounded functions on $S$. For $s \in S$, define $\varrho_{s}: S \rightarrow S$ by $\varrho_{s}(t)=t s$ for $t \in S$. Define $R_{s}$ : $\ell^{\infty}(S) \rightarrow \ell^{\infty}(S)$ by

$$
R_{s}(f)=f \circ \varrho_{s} \quad\left(s \in S, f \in \ell^{\infty}(S)\right),
$$


Let $C(S) \subseteq \ell^{\infty}(S)$ be the space of continuous, bounded functions on $S$. For $f \in \ell^{\infty}(S)$, we say that $f$ is weakly almost periodic, denoted by $f \in$ $\mathcal{W} \mathcal{A} \mathcal{P}(S)$, when $f \in C(S)$, and $R_{S}(f):=\left\{R_{s}(f): s \in S\right\}$ is relatively weakly compact in $\ell^{\infty}(S)$. As noted in [4, Chapter 4], if $S_{\mathrm{d}}$ denotes the semigroup $S$ with the discrete topology, then $\mathcal{W} \mathcal{A} \mathcal{P}(S)=\mathcal{W} \mathcal{A} \mathcal{P}\left(S_{\mathrm{d}}\right) \cap C(S)$.

THEOREM 7.1. Let $S$ be a semitopological semigroup, and let $f \in C(S)$. Then $f \in \mathcal{W} \mathcal{A P}(S)$ if and only if

$$
\lim _{n \rightarrow \infty} \lim _{m \rightarrow \infty} f\left(s_{m} t_{n}\right)=\lim _{m \rightarrow \infty} \lim _{n \rightarrow \infty} f\left(s_{m} t_{n}\right)
$$

whenever $\left(s_{m}\right)$ and $\left(t_{n}\right)$ are sequences of distinct elements of $S$, and the iterated limits exist. Then $\mathcal{W} \mathcal{A P}(S)$ is a translation invariant sub-C ${ }^{*}$-algebra of $\ell^{\infty}(S)$ which contains the constant functions.

We shall now concentrate on the case when $S$ is discrete. We turn $\ell^{1}(S)$ into a Banach algebra with the convolution product in the usual way (see [9] for further details about such algebras). Using the double limit criterion above, it is a simple matter to check that $\mathcal{W} \mathcal{A P}(S)=\operatorname{WAP}\left(\ell^{\infty}(S)\right)$ where we treat $\ell^{\infty}(S)$ as an $\ell^{1}(S)$-bimodule. Thus, we see that the Arens products drop to a well-defined product on $\mathcal{W} \mathcal{A P}(S)^{\prime}$ turning $\mathcal{W} \mathcal{A P}(S)^{\prime}$ into a dual Banach algebra.

Similar conclusions can be drawn when $S$ is a locally compact group (see [9, Chapter 7] for example) but the arguments involved are more intricate. For example, in [36], it is shown that $\operatorname{WAP}\left(L^{\infty}(G)\right)=\mathcal{W} \mathcal{A} \mathcal{P}(G)$ for a locally compact group $G$. The argument there seems to rely upon certain properties of groups, and it is far from clear that an analogous result will hold in situations where $L^{1}(T)$ makes sense for a topological semigroup $T$. See [21] for recent progress in the study of when $L^{1}(T)$ makes sense for such a semigroup $T$.

Let $S^{\mathcal{W} \mathcal{A P}}$ be the character space of the $\mathrm{C}^{*}$-algebra $\mathcal{W} \mathcal{A} \mathcal{P}(S)$. Define a map $\epsilon: S \rightarrow S^{\mathcal{W} \mathcal{A P}}$ by letting $\epsilon(s)$ be point evaluation at $s \in S$. We may check that the product on $\mathcal{W} \mathcal{A} \mathcal{P}(S)^{\prime}$ restricts to the character space $S^{\mathcal{W} \mathcal{A P}}$, so that $S^{\mathcal{W} \mathcal{A P}}$ becomes a semigroup, and $\epsilon$ becomes a homomorphism.

For a semitopological semigroup $S$, a semitopological semigroup compactification of $S$ is a pair $(\psi, T)$ where $T$ is a compact, Hausdorff, semitopological semigroup, and $\psi: S \rightarrow T$ is a continuous homomorphism with dense range. We do not require that $\psi$ be injective, so this differs from the notion of a compactification of a topological group. A semitopological semigroup compactification $(\psi, T)$ is universal if whenever $R$ is another compact semitopological semigroup and $\phi: S \rightarrow R$ is a homomorphism, $\phi$ factors through $(\psi, T)$. Clearly any two universal compactifications are isomorphic.

THEOREM 7.2. With notation as above, $\left(\epsilon, S^{\mathcal{W} \mathcal{A P}}\right)$ is a universal semitopological semigroup compactification of $S$. 
We shall henceforth drop the $\epsilon$ and write $S^{\mathcal{W A P}}$ for the $\mathcal{W} \mathcal{A P}$-compactification of $S$. Notice that $\mathcal{W} \mathcal{A P}(S)$ is isomorphic to $C\left(S^{\mathcal{W} \mathcal{A P}}\right)$ and so $\mathcal{W} \mathcal{A} \mathcal{P}(S)^{\prime}$ is isomorphic to $M\left(S^{\mathcal{W} \mathcal{A P}}\right)$. We may check that the product on $\mathcal{W} \mathcal{A} \mathcal{P}(S)^{\prime}$ agrees with the natural convolution product on $M\left(S^{\mathcal{W} \mathcal{A P}}\right)$.

EXAMPLE 7.3. Let $S$ be a discrete semigroup. Then $\ell^{1}(S)$ is a Banach algebra, and $c_{0}(S)$ is a predual for the Banach space $\ell^{1}(S)$. We see that the product is weak*-continuous if and only if $S$ is a weakly cancellative discrete semigroup, that is, the left and right actions are finite-to-one maps. This follows by an easy calculation: see [11, Proposition 5.1] for example.

EXAMPle 7.4. Let $S=(\mathbb{N}, \max )$, so that $\ell^{1}(S)$ is a dual Banach algebra with predual $c_{0}(S)$. Then $S^{\mathcal{W} \mathcal{A P}}$ is a compact semitopological semigroup containing $S$ as a dense subsemigroup. We may check that $S^{\mathcal{W} \mathcal{A P}}$ is equal to $S$ with an adjoined zero, denoted by $\infty$, which satisfies $\infty n=n \infty=\infty$ for $n \in S^{\mathcal{W} \mathcal{A P}}$. The topology is then simply the one-point compactification.

EXAMPLE 7.5. Let $S=(\mathbb{N}, \min )$, so that $\ell^{1}(S)$ is a Banach algebra, but as $S$ is not weakly cancellative, $\ell^{1}(S)$ is not a dual Banach algebra with respect to $c_{0}(S)$. We may check that $S^{\mathcal{W} \mathcal{A P}}$ is equal to $S$ with an adjoined identity, denoted again by $\infty$, so that $\infty n=n \infty=n$ for $n \in S^{\mathcal{W} \mathcal{A P}}$. The topology is again the one-point compactification.

For example, the $\mathcal{W} \mathcal{A} \mathcal{P}$-compactification of $(\mathbb{Z},+)$ is a much more mysterious object.

7.1. Injectivity and semigroup algebras. We now apply the idea of injectivity to some semigroup algebras. As will be seen, the results we get are rather simple, while the necessary Banach space machinery is fairly involved, all suggesting that we really need some further tools to make this approach worthwhile.

Let $E$ be a Banach space with a normalised basis $\left(e_{n}\right)$. See [24] for further details on bases in Banach spaces. For each $n$, there is a linear functional $e_{n}^{*} \in E^{\prime}$ given by $\left\langle e_{n}^{*}, \sum_{i} x_{i} e_{i}\right\rangle=x_{n}$. By a standard renorming of $E$, we may suppose that the projection onto the linear span of $\left(e_{i}\right)_{i=1}^{n}$ is norm-decreasing.

Let $S=(\mathbb{N}, \min )$. There is then a natural representation $\pi: \ell^{1}(S) \rightarrow$ $\mathcal{B}(E)$ given by

$$
\pi\left(\delta_{n}\right)\left(e_{m}\right)=\left\{\begin{array}{ll}
e_{m}, & m \leq n, \\
0, & m>n,
\end{array} \quad(n, m \in \mathbb{N})\right.
$$

and linearity. That is, $\pi\left(\delta_{n}\right)$ is the projection onto the linear span of the first $n$ basis elements. Let $\mathcal{A}=\pi\left(\ell^{1}(S)\right) \subseteq \mathcal{B}(E)$.

Each element of $\mathcal{B}(E)$ has a natural representation as a matrix with respect to the basis $\left(e_{n}\right)$. We claim that $\mathcal{A}^{\mathrm{c}}$ is just the diagonal matrices in $\mathcal{B}(E)$. Clearly a diagonal matrix is in $\mathcal{A}^{\mathrm{c}}$, while conversely, since $\pi(n)-$ 
$\pi(n-1)=e_{n}^{*} \otimes e_{n}$, we see that for $T \in \mathcal{A}^{\mathrm{c}}$,

$T\left(e_{n}^{*} \otimes e_{n}\right)\left(e_{m}\right)=\delta_{n, m} T\left(e_{n}\right)=\left(e_{n}^{*} \otimes e_{n}\right) T\left(e_{m}\right)=\left\langle e_{n}^{*}, T\left(e_{m}\right)\right\rangle e_{n} \quad(n, m \in \mathbb{N}) ;$

here $\delta$ denotes the Kronecker delta. We hence see that $T\left(e_{n}\right) \in \mathbb{C} e_{n}$ for each $n \in \mathbb{N}$, as required.

We now claim that if there exists a quasi-expectation $\mathcal{Q}: \mathcal{B}(E) \rightarrow \mathcal{A}^{\mathrm{c}}$, then $\mathcal{Q}$ must be the canonical projection onto the diagonal of $\mathcal{B}(E)$. Let $n, m \in \mathbb{N}$, let $a=e_{n}^{*} \otimes e_{n}, b=e_{m}^{*} \otimes e_{m} \in \mathcal{A}^{\mathrm{c}}$ and let $T \in \mathcal{B}(E)$. Then

$$
\left\langle e_{n}^{*}, T\left(e_{m}\right)\right\rangle \mathcal{Q}\left(e_{m}^{*} \otimes e_{n}\right)=\mathcal{Q}(a T b)=a \mathcal{Q}(T) b=\left\langle e_{n}^{*}, \mathcal{Q}(T)\left(e_{m}\right)\right\rangle e_{m}^{*} \otimes e_{n},
$$

so that, if $n \neq m$, then $e_{m}^{*} \otimes e_{n} \notin \mathcal{A}^{\mathrm{c}}$, so $\left\langle e_{n}^{*}, \mathcal{Q}(T)\left(e_{m}\right)\right\rangle=0$. Thus $\mathcal{Q}(T)\left(e_{n}\right) \in \mathbb{C} e_{n}$ for each $n$, and we see that

$$
\left\langle e_{n}^{*}, T\left(e_{n}\right)\right\rangle e_{n}^{*} \otimes e_{n}=\left\langle e_{n}^{*}, T\left(e_{n}\right)\right\rangle \mathcal{Q}\left(e_{n}^{*} \otimes e_{n}\right)=\left\langle e_{n}^{*}, \mathcal{Q}(T)\left(e_{n}\right)\right\rangle e_{n}^{*} \otimes e_{n},
$$

and hence $\mathcal{Q}(T)\left(e_{n}\right)=\left\langle e_{n}^{*}, T\left(e_{n}\right)\right\rangle$, as required.

Theorem 7.6. Let $S=(\mathbb{N}, \min )$. Then $\operatorname{WAP}\left(\ell^{1}(S)^{\prime}\right)^{\prime}$ is not Connesamenable.

Proof. By Proposition 6.15 (but really by Proposition 6.3) it suffices to find a reflexive Banach space with a basis $\left(e_{n}\right)$ such that the canonical projection from $\mathcal{B}(E)$ onto its diagonal is not bounded.

Following [24, Proposition 2.b.11], there exists a sequence $\left(\beta_{n}\right)_{n \in \mathbb{N}}$ of positive reals tending to infinity such that, for each $n \in \mathbb{N}$, we can find a Banach space $H_{n}$ such that:

1. there is an isomorphism $\phi_{n}: H_{n} \rightarrow \ell_{2 n}^{2}$ (that is, the space $\mathbb{C}^{2 n}$ with the usual Euclidean norm) such that $\left\|\phi_{n}\right\|\left\|\phi_{n}^{-1}\right\| \leq K$ for some absolute constant $K>0$

2. $H_{n}$ has a normalised basis (in our sense, as above) $\left(e_{k}\right)_{k=1}^{2 n}$;

3. there exists $\left(a_{k}\right)_{k=1}^{2 n} \subseteq \mathbb{C}$ with

$$
\left\|\sum_{k=1}^{n} a_{2 k-1} e_{2 k-1}\right\| \geq \beta_{n}\left\|\sum_{k=1}^{2 n} a_{k} e_{k}\right\| .
$$

Let $K_{n}$ be the subspace spanned by $\left(e_{2 k-1}\right)_{k=1}^{n}$. As $H_{n}$ is isomorphic to $\ell_{2 n}^{2}$, let $P_{n}$ be the orthogonal projection onto $K_{n}$ (pulled back by $\phi_{n}$ ), so that $\left\|P_{n}\right\| \leq K$. Then $P_{n}\left(e_{k}\right)=e_{k}$ when $k$ is odd, while for even $k$, clearly $\left\langle e_{k}^{*}, P_{n}\left(e_{k}\right)\right\rangle=0$, as $P_{n}\left(e_{k}\right) \in K_{n}$. Let $\mathcal{Q}_{n}$ be the canonical projection of $\mathcal{B}\left(H_{n}\right)$ onto its diagonal, so that $\mathcal{Q}_{n}\left(P_{n}\right)=\sum_{k=1}^{n} e_{2 k-1}^{*} \otimes e_{2 k-1}$. Then let $x=\sum_{k=1}^{2 n} a_{k} e_{k}$, so that

$$
\left\|\mathcal{Q}_{n}\left(P_{n}\right)(x)\right\|=\left\|\sum_{k=1}^{n} a_{2 k-1} e_{2 k-1}\right\| \geq \beta_{n}\|x\|,
$$

so $\left\|\mathcal{Q}_{n}\left(P_{n}\right)\right\| \geq \beta_{n}$, and hence $\left\|\mathcal{Q}_{n}\right\| \geq K^{-1} \beta_{n}$. 
Let $E$ be the $\ell^{2}$-direct sum of the $H_{n}$, so that if $\mathcal{Q}$ is the canonical projection from $\mathcal{B}(E)$ onto its diagonal, we see that $\|\mathcal{Q}\| \geq \beta_{n}$ for every $n$, which gives a contradiction. We hence see that $\operatorname{WAP}\left(\ell^{1}(S)^{\prime}\right)^{\prime}$ is not Connesamenable when $S=(\mathbb{N}, \min )$.

Notice that when $\pi: \ell^{1}(\mathbb{N}, \min ) \rightarrow \mathcal{B}(E)$ is a representation, then $\pi$ is a well-defined linear operator $\ell^{1}(\mathbb{N}, \max ) \rightarrow \mathcal{B}(E)$, and it is easily checked that the map $\ell^{1}(\mathbb{N}, \max ) \rightarrow \mathcal{B}(E), \delta_{n} \mapsto I_{E}-\pi(n)$, is a homomorphism. The commutant for either of these maps is equal, and hence we see that $\operatorname{WAP}\left(\ell^{1}(\mathbb{N}, \max )^{\prime}\right)^{\prime}$ is also not Connes-amenable.

It seems quite possible that various interesting Banach spaces will be generated by starting with a complex semigroup $S$ for which we know that $\operatorname{WAP}\left(\ell^{1}(S)^{\prime}\right)^{\prime}$ is not Connes-amenable, and then looking at the representations generated by our results.

We now briefly mention how to use the notion of injectivity to show that a dual Banach algebra is Connes-amenable. For example, let $\pi: \ell^{1}(\mathbb{Z}) \rightarrow \mathcal{B}(E)$ be a representation on some reflexive Banach space $E$. We construct a quasiexpectation $\mathcal{Q}: \mathcal{B}(E) \rightarrow \pi\left(\ell^{1}(\mathbb{Z})\right)^{\mathrm{c}}$ by

$$
\mathcal{Q}(T)=\lim _{n \rightarrow \infty} \frac{1}{n} \sum_{k=1}^{n} \pi\left(\delta_{-k}\right) T \pi\left(\delta_{k}\right),
$$

where the limit is in the weak*-topology on $\mathcal{B}(E)$. Then, for $m \geq 0$,

$$
\begin{aligned}
\| \pi\left(\delta_{m}\right) \mathcal{Q}(T) & -\mathcal{Q}(T) \pi\left(\delta_{m}\right) \| \\
& =\lim _{n \rightarrow \infty}\left\|\frac{1}{n} \sum_{k=1}^{n}\left(\pi\left(\delta_{m-k}\right) T \pi\left(\delta_{k}\right)-\pi\left(\delta_{-k}\right) T \pi\left(\delta_{k+m}\right)\right)\right\| \\
& =\lim _{n \rightarrow \infty}\left\|\frac{1}{n} \sum_{k=1}^{m} \pi\left(\delta_{m-k}\right) T \pi\left(\delta_{k}\right)\right\| \leq \lim _{n \rightarrow \infty} \frac{m}{n}\|T\|\|\pi\|^{2}=0,
\end{aligned}
$$

and a similar argument holds when $m<0$. Thus $\mathcal{Q}$ is a projection onto $\pi\left(\ell^{1}(\mathbb{Z})\right)^{\mathrm{c}}$, and it is simple to verify that $\mathcal{Q}$ is a quasi-projection. Of course, in this argument, we have really used, rather directly, the fact that $\mathbb{Z}$ is an amenable group.

It seems more natural and profitable to study the Connes-amenability of algebras of operators via injectivity, something we hope to pursue in future research.

8. Tensor products. In this section, we shall sketch some ideas about tensor products of Banach algebras which behave well with respect to weakly almost periodic functionals, and then go on to give a theory of tensor products of dual Banach algebras.

We start by sketching some results on tensor products of Banach spaces. 
We follow the notation used in Banach space theory, namely that found in [31], and in [13] (except where this clashes with notation in [31]). Note that this notation is different from that found in [32], for example.

Let $E$ and $F$ be Banach spaces. We have previously defined the projective tensor product $E \widehat{\otimes} F$. The injective tensor norm, $\epsilon$, is defined by, for $\tau=$ $\sum_{k=1}^{n} x_{k} \otimes y_{k} \in E \otimes F$,

$$
\epsilon(\tau)=\sup \left\{\left|\sum_{k=1}^{n}\left\langle\mu, x_{k}\right\rangle\left\langle\lambda, y_{k}\right\rangle\right|: \mu \in E^{\prime}, \lambda \in F^{\prime},\|\mu\|=\|\lambda\|=1\right\} .
$$

We write $E \check{\otimes} F$ for the completion of $E \otimes F$ with respect to $\epsilon$. Notice that if we identify $E \otimes F$ with a subspace of the finite rank operators $\mathcal{F}\left(E^{\prime}, F\right)$, then $\epsilon$ is the norm induced by the operator norm under this identification.

Let $\alpha$ be a norm on $E \otimes F$. Then $\alpha$ is a reasonable crossnorm when $\epsilon(\tau) \leq$ $\alpha(\tau) \leq \pi(\tau)$ for each $\tau \in E \otimes F$. In this case, clearly $\alpha(x \otimes y)=\|x\|\|y\|$ for $x \in E$ and $y \in F$. We write $E \widehat{\otimes}_{\alpha} F$ for the completion of $E \otimes F$ with respect to $\alpha$.

A uniform crossnorm is an assignment, to each pair of Banach spaces $E$ and $F$, of a norm $\alpha$ with the following mapping property. Let $G$ and $H$ be Banach spaces, let $T \in \mathcal{B}(E, G)$ and $S \in \mathcal{B}(F, H)$, and define $T \otimes S$ : $E \otimes F \rightarrow G \otimes H$ by $(T \otimes S)(x \otimes y)=T(x) \otimes S(y)$ and linearity. Then we insist that $T \otimes S$ extends by continuity to a bounded linear map $E \widehat{\otimes}_{\alpha} F \rightarrow G \widehat{\otimes}_{\alpha} H$ with norm $\|T\|\|S\|$. In the special case when this mapping property holds with $E=G, F=H$ and $\alpha$ a reasonable crossnorm on $E \otimes F$, we say (in a non-standard way) that $\alpha$ is a quasi-uniform crossnorm.

We define an action of $E^{\prime} \otimes F^{\prime}$ on $E \otimes F$ by setting

$$
\langle\mu \otimes \lambda, x \otimes y\rangle=\langle\mu, x\rangle\langle\lambda, y\rangle \quad\left(x \in E, y \in F, \mu \in E^{\prime}, \lambda \in F^{\prime}\right),
$$

and extending by linearity. We define the dual norm $\alpha^{\mathrm{s}}$ on $E^{\prime} \otimes F^{\prime}$ by

$$
\alpha^{\mathrm{S}}(\sigma)=\sup \{|\langle\sigma, \tau\rangle|: \tau \in E \otimes F, \alpha(\tau) \leq 1\} \quad\left(\sigma \in E^{\prime} \otimes F^{\prime}\right) .
$$

Then it may be checked that $\alpha^{\mathrm{s}}$ is a reasonable crossnorm when $\alpha$ is, and similarly for uniform crossnorms. When $E$ and $F$ are reflexive, and $\alpha$ is a quasi-uniform crossnorm, then $\alpha^{\mathrm{s}}$ is also quasi-uniform. Then $\pi^{\mathrm{s}}=\epsilon$ for all Banach spaces, but $\epsilon^{\mathrm{s}}=\pi$ only in special cases.

A tensor norm is then a uniform crossnorm which respects finite-dimensional subspaces in a certain sense. We shall not have use of this idea, but do note that many of the norms we construct in this section are not as well-behaved as those studied in [31] and [13].

As explained before, it is standard that $(E \widehat{\otimes} F)^{\prime}=\mathcal{B}\left(E, F^{\prime}\right)$ for Banach spaces $E$ and $F$. For any reasonable crossnorm $\alpha$ on $E \otimes F$, as the formal inclusion map $E \widehat{\otimes} F \rightarrow E \widehat{\otimes}_{\alpha} F$ is norm-decreasing, we may use the adjoint to identify $\left(E \widehat{\otimes}_{\alpha} F\right)^{\prime}$ with a subspace of $\mathcal{B}\left(E, F^{\prime}\right)$, together with the dual 
norm. For example, the dual of $E \check{\otimes} F$ is $\mathcal{I}\left(E, F^{\prime}\right)$, the integral operators from $E$ to $F^{\prime}$ (see [31, Chapter 3] for further details).

As explained above, $\epsilon^{\mathrm{s}}=\pi$ only in special cases, which means that in general, the natural map $E^{\prime} \widehat{\otimes} F^{\prime} \rightarrow(E \check{\otimes} F)^{\prime}=\mathcal{I}\left(E, F^{\prime}\right)$ is only norm-decreasing. An important special case is when $E$ or $F$ has the metric approximation property, in which case $E^{\prime} \widehat{\otimes} F^{\prime}$ is, isometrically, a closed subspace of $\mathcal{I}\left(E, F^{\prime}\right)$. See [31, Section 4] for further details. Another way to state this result is to consider the natural map from $E^{\prime} \widehat{\otimes} F^{\prime}$ to $\mathcal{B}\left(E, F^{\prime}\right)$, which has range $\mathcal{N}\left(E, F^{\prime}\right)$, the nuclear operators. Thus $\mathcal{N}\left(E, F^{\prime}\right)$ is closed in $\mathcal{I}\left(E, F^{\prime}\right)$ when $E$ or $F$ has the metric approximation property.

ExAMPLE 8.1. Let $X$ and $Y$ be locally compact Hausdorff spaces. Then $C_{0}(X) \check{\otimes} C_{0}(Y)=C_{0}(X \times Y)$ under the obvious identification (see [31, Section 3.2]). As $C(X)$ has the metric approximation property, we find that $M(X) \widehat{\otimes} M(Y)$ forms a closed subspace of $M(X \times Y)$. We shall see below that we can fail to have equality.

Similarly, let $\mu$ and $\nu$ be measures. Then $L^{1}(\mu) \widehat{\otimes} L^{1}(\nu)=L^{1}(\mu \times \nu)$ under the obvious identification (see [31, Chapter 2]).

Hence, if $X$ and $Y$ are discrete sets, then $c_{0}(X) \otimes c_{0}(Y)=c_{0}(X \times Y)$ and $c_{0}(X)^{\prime} \widehat{\otimes} c_{0}(Y)^{\prime}=\ell^{1}(X) \widehat{\otimes} \ell^{1}(Y)=\ell^{1}(X \times Y)=c_{0}(X \times Y)^{\prime}$.

\subsection{Tensor products of algebras}

Definition 8.2. Let $\mathcal{A}$ and $\mathcal{B}$ be Banach algebras, and define an algebra product on $\mathcal{A} \otimes \mathcal{B}$ by $(a \otimes b)(c \otimes d)=a c \otimes b d$, and linearity, for $a, c \in \mathcal{A}$ and $b, d \in \mathcal{B}$. Then an algebra crossnorm on $\mathcal{A} \otimes \mathcal{B}$ is a reasonable crossnorm $\alpha$ such that $\mathcal{A} \widehat{\otimes}_{\alpha} \mathcal{B}$ becomes a Banach algebra.

Notice that the projective tensor norm is always an algebra crossnorm, but that the injective tensor norm may not be (indeed, it is shown in [6] that only four of Grothendieck's fourteen "natural" tensor norms are always algebra crossnorms).

We have shown that WAP algebras are isomorphic (but maybe not isometric) to closed subalgebras of $\mathcal{B}(E)$ for suitable reflexive $E$. In this section, it is convenient to suppose that a WAP algebra $\mathcal{A}$ is isometric to a closed subalgebra of $\mathcal{B}(E)$ for suitable $E$. This can clearly be achieved by considering a suitable renorming of $\mathcal{A}$.

Definition 8.3. Let $\mathcal{A}$ and $\mathcal{B}$ be WAP algebras. A WAP-crossnorm on $\mathcal{A} \otimes \mathcal{B}$ is an algebra crossnorm $\alpha$ such that, if we form the natural chain of natural inclusion maps $\operatorname{WAP}\left(\mathcal{A}^{\prime}\right) \otimes \operatorname{WAP}\left(\mathcal{B}^{\prime}\right) \subseteq \mathcal{A}^{\prime} \otimes \mathcal{B}^{\prime} \subseteq \mathcal{A}^{\prime} \widehat{\otimes}_{\alpha^{\mathrm{s}}} \mathcal{B}^{\prime} \subseteq$ $\left(\mathcal{A} \widehat{\otimes}_{\alpha} \mathcal{B}\right)^{\prime}$, then we actually map into $\operatorname{WAP}\left(\left(\mathcal{A} \widehat{\otimes}_{\alpha} \mathcal{B}\right)^{\prime}\right)$, and that furthermore, $\operatorname{WAP}\left(\mathcal{A}^{\prime}\right) \otimes \operatorname{WAP}\left(\mathcal{B}^{\prime}\right)$ is norming for $\mathcal{A} \widehat{\otimes}_{\alpha} \mathcal{B}$.

We shall see that the condition on $\operatorname{WAP}\left(\mathcal{A}^{\prime}\right) \otimes \operatorname{WAP}\left(\mathcal{B}^{\prime}\right)$ is natural when we come to consider dual Banach algebras. 
EXAMPLE 8.4. Let $K$ and $L$ be compact Hausdorff spaces, and consider the injective tensor product $C(K) \check{\otimes} C(L)=C(K \times L)$. As these are commutative $\mathrm{C}^{*}$-algebras, they are Arens regular, and so $\mathrm{WAP}\left(C(K)^{\prime}\right)=M(K)$, $\mathrm{WAP}\left(C(L)^{\prime}\right)=M(L)$, and hence $M(K) \otimes M(L) \subseteq \mathrm{WAP}(C(K \times L))$. As explained above, we induce the projective tensor norm on $M(K) \otimes M(L)$ by embedding it into $C(K \times L)^{\prime}=M(K \times L)$. Then $(M(K) \widehat{\otimes} M(L))^{\prime}=$ $\mathcal{B}\left(M(K), M(L)^{\prime}\right)$, and so we get the chain of isometric inclusions

$$
\begin{aligned}
C(K) \check{\otimes} C(L) & \subseteq \mathcal{A}(M(K), C(L)) \subseteq \mathcal{A}\left(M(K), M(L)^{\prime}\right) \\
& \subseteq \mathcal{B}\left(M(K), M(L)^{\prime}\right) .
\end{aligned}
$$

Thus $M(K) \otimes M(L)$ is norming for $C(K \times L)$. We could also see this directly by considering point masses in $M(K)$ and $M(L)$.

However, $M(K) \otimes M(L)$ is not in general dense in $M(K \times L)$. Let $L$ be a compact Hausdorff space such that $M(L)$ does not have the RadonNikodym property (see [14] for what this technical condition is). For example, [14, Chapter VII] shows that this holds when there is a separable subspace of $C(L)$ without a separable dual. As indicated in [14, Chapter VI, Corollary 6], there then exists an integral, non-nuclear operator from $C(K)$ to $M(L)$ whenever $K$ contains a perfect subset (that is, a closed subset with no isolated points). For example, let $\mathbb{T}=\{z \in \mathbb{C}:|z|=1\}$, so that $\mathbb{T}$ is perfect, and $M(\mathbb{T})$ does not have the Radon-Nikodým property. Thus $M(\mathbb{T}) \otimes M(\mathbb{T})$ is not dense in $(C(\mathbb{T}) \check{\otimes} C(\mathbb{T}))^{\prime}$.

EXAMPLE 8.5. Let $S$ and $T$ be discrete semigroups, so that $\operatorname{WAP}\left(\ell^{1}(S)^{\prime}\right)$ $=C\left(S^{\mathcal{W} \mathcal{A P}}\right)$. Then $\ell^{1}(S) \widehat{\otimes} \ell^{1}(T)=\ell^{1}(S \times T)$ as a Banach algebra, and so $\mathrm{WAP}\left(\ell^{1}(S \times T)^{\prime}\right)=C\left((S \times T)^{\mathcal{W} \mathcal{A P}}\right)$. We claim that $(S \times T)^{\mathcal{W} \mathcal{A P}}=S^{\mathcal{W} \mathcal{A P}} \times$ $T^{\mathrm{WAP}}$, which follows easily by the universality property of $(S \times T)^{\mathcal{W} \mathcal{A} \mathcal{P}}$. We then see that

$$
\begin{aligned}
\operatorname{WAP}\left(\ell^{1}(S)^{\prime}\right) \check{\otimes} \operatorname{WAP}\left(\ell^{1}(T)^{\prime}\right) & =C\left(S^{\mathcal{W} \mathcal{A P}}\right) \check{\otimes} C\left(T^{\mathcal{W} \mathcal{A P}}\right) \\
& =C\left(S^{\mathcal{W} \mathcal{A P}} \times T^{\mathcal{W} \mathcal{A P}}\right)=C\left((S \times T)^{\mathcal{W A P}}\right) \\
& =\operatorname{WAP}\left(\left(\ell^{1}(S) \widehat{\otimes} \ell^{1}(T)\right)^{\prime}\right),
\end{aligned}
$$

so certainly $\operatorname{WAP}\left(\ell^{1}(S)^{\prime}\right) \otimes \operatorname{WAP}\left(\ell^{1}(T)^{\prime}\right)$ is dense in $\operatorname{WAP}\left(\left(\ell^{1}(S) \widehat{\otimes} \ell^{1}(T)\right)^{\prime}\right)$.

As before, this argument also works for general locally compact groups $G$ and $H$.

EXAMPLE 8.6. Let $S$ and $T$ be discrete weakly cancellative semigroups, so that $c_{0}(S) \check{\otimes} c_{0}(T)=c_{0}(S \times T)$, and hence $\left(c_{0}(S) \check{\otimes} c_{0}(T)\right)^{\prime}=\ell^{1}(S \times T)=$ $\ell^{1}(S) \widehat{\otimes} \ell^{1}(T)$ is a dual Banach algebra.

Similarly, let $S$ and $T$ be locally compact groups, so that $M(S)$ has the predual $C_{0}(S)$, and similarly for $M(T)$ (see [30]). Then, as above, we see that $M(S) \widehat{\otimes} M(T)$ is a closed, norming (and hence weak*-dense) subspace of $M(S \times T)=C_{0}(S \times T)^{\prime}=\left(C_{0}(S) \ddot{\otimes} C_{0}(T)\right)^{\prime}$. 
Proposition 8.7. Let $\mathcal{A}$ and $\mathcal{B}$ be Banach algebras. Then the natural map $\operatorname{WAP}\left(\mathcal{A}^{\prime}\right) \otimes \operatorname{WAP}\left(\mathcal{B}^{\prime}\right) \rightarrow \mathcal{A}^{\prime} \widehat{\otimes}_{\pi^{s}} \mathcal{B}^{\prime}$ maps into $\operatorname{WAP}\left((\mathcal{A} \widehat{\otimes} \mathcal{B})^{\prime}\right)$. If $\mathcal{A}$ and $\mathcal{B}$ are Arens regular and one has the metric approximation property, then $\pi$ is a WAP-crossnorm on $\mathcal{A} \otimes \mathcal{B}$.

Proof. Recall that $\pi^{\mathrm{s}}=\epsilon$, and let $\mu \in \operatorname{WAP}\left(\mathcal{A}^{\prime}\right)$ and $\lambda \in \operatorname{WAP}\left(\mathcal{B}^{\prime}\right)$. Then there exists a reflexive Banach space $E$ and maps $S_{\mu}: \mathcal{A} \rightarrow E$ and $T_{\mu}: E \rightarrow$ $\mathcal{A}^{\prime}$ such that $T_{\mu} S_{\mu}=L_{\mu}$, that is, $T_{\mu} S_{\mu}(a)=a \cdot \mu$ for $a \in \mathcal{A}$. Similarly there exists a reflexive Banach space $F$ and maps $S_{\lambda}$ and $T_{\lambda}$. We shall see below that there exists a uniform crossnorm $\alpha$ such that $E \widehat{\otimes}_{\alpha} F$ is reflexive. As $\epsilon \leq \alpha \leq \pi$, we see that we can factor the map $L_{\mu} \otimes L_{\lambda}: \mathcal{A} \widehat{\otimes} \mathcal{B} \rightarrow \mathcal{A}^{\prime} \check{\otimes} \mathcal{B}^{\prime}$ as

$$
\mathcal{A} \widehat{\otimes} \mathcal{B} \stackrel{S_{\mu} \otimes S_{\lambda}}{\longrightarrow} E \widehat{\otimes} F \rightarrow E \widehat{\otimes}_{\alpha} F \stackrel{T_{\mu} \otimes T_{\lambda}}{\longrightarrow} \mathcal{A}^{\prime} \widehat{\otimes}_{\alpha} \mathcal{B}^{\prime} \rightarrow \mathcal{A}^{\prime} \check{\otimes} \mathcal{B}^{\prime}
$$

and so $L_{\mu} \otimes L_{\lambda}=L_{\mu \otimes \lambda}$ is weakly compact. By linearity, the argument is complete.

Suppose now that $\mathcal{A}$ has the metric approximation property. By [31, Theorem 4.14], the canonical map $\mathcal{A} \widehat{\otimes} \mathcal{B} \rightarrow\left(\mathcal{A}^{\prime} \check{\otimes}^{\prime}\right)^{\prime}$ is an isometry, so that $\mathcal{A}^{\prime} \check{\otimes} \mathcal{B}^{\prime}$ is norming for $\mathcal{A} \widehat{\otimes} \mathcal{B}$. As $\mathcal{A}$ and $\mathcal{B}$ are Arens regular, we see that $\pi$ is indeed a WAP-crossnorm on $\mathcal{A} \otimes \mathcal{B}$. The case for $\mathcal{B}$ follows by symmetry.

EXAMPLE 8.8. Let $c=C\left(\mathbb{N}_{\infty}\right)$ be the space of convergent sequences, where $\mathbb{N}_{\infty}$ is the one-point compactification of $\mathbb{N}$. Then $c^{\prime}=\ell^{1}$ naturally, and so $(c \widehat{\otimes} c)^{\prime}=\mathcal{B}\left(c, \ell^{1}\right)=\mathcal{A}\left(c, \ell^{1}\right)=\ell^{1} \check{\otimes} \ell^{1}$. Then $\pi$ is a WAP-crossnorm for $c \otimes c$ as $c$ is Arens regular. In fact, $c \widehat{\otimes} c$ is Arens regular.

For general compact Hausdorff spaces $K$ and $L$, by the above proposition, we see that $\pi$ is a WAP-crossnorm on $C(K) \otimes C(L)$. However, as noted in [34], when $G$ is a compact group, the algebra $C(G) \widehat{\otimes} C(G)$ contains a copy of $A(G)$, the Fourier algebra of $G$, so that $C(G) \widehat{\otimes} C(G)$ is not Arens regular. Hence $M(G) \otimes M(G) \subseteq(C(G) \widehat{\otimes} C(G))^{\prime}$ cannot be dense.

See [35] for details about when $\mathcal{A} \widehat{\otimes} \mathcal{B}$ is Arens regular, but bear in mind the correction [34].

It seems that in general, $\operatorname{WAP}\left(\mathcal{A}^{\prime}\right) \check{\otimes} \mathrm{WAP}\left(\mathcal{B}^{\prime}\right)$ need not be norming for $\mathcal{A} \widehat{\otimes} \mathcal{B}$. Also, we see no way to adapt the above proof to the case $\mathcal{A} \widehat{\otimes}_{\beta} \mathcal{B}$ for an arbitrary algebra crossnorm $\beta$.

Proposition 8.9. Given reflexive Banach spaces $E$ and $F$, there exists a tensor norm $\alpha$ on $E \otimes F$ such that $E \widehat{\otimes}_{\alpha} F$ is reflexive, and such that $E^{\prime} \otimes F^{\prime}$ is dense in $\left(E \widehat{\otimes}_{\alpha} F\right)^{\prime}$.

Proof. For example, for $1<p<\infty$, let $g_{p}$ and $d_{p}$ be the Chevet-Saphar tensor norms, as defined in [31, Chapter 6] or [1]. Then, by [1, Corollary 3.2], we find that $E \widehat{\otimes}_{g_{p}} F$ is reflexive whenever $E$ and $F$ are, and similarly for $d_{p}$. 
Furthermore, $E^{\prime} \otimes F^{\prime}$ is indeed dense in $\left(E \widehat{\otimes}_{g_{p}} F\right)^{\prime}$ and $\left(E \widehat{\otimes}_{d_{p}} F\right)^{\prime}$. As $E^{\prime} \otimes F^{\prime}$ is dense in $\left(E \widehat{\otimes}_{\alpha} F\right)^{\prime}$, we see that $\left(E \widehat{\otimes}_{\alpha} F\right)^{\prime}=E^{\prime} \widehat{\otimes}_{\alpha^{s}} F^{\prime}$.

REMARK 8.10. One disadvantage of the Chevet-Saphar tensor norms is that they are not symmetric, in that $E \widehat{\otimes}_{g_{p}} F$ is not in general isomorphic to $F \widehat{\otimes}_{g_{p}} E$ (in contrast to the injective or projective tensor norms). However, $d_{2}$ and $g_{2}$ behave rather nicely, in that $d_{2}^{\mathrm{s}}=g_{2}, g_{2}^{\mathrm{s}}=d_{2}$, and $H \widehat{\otimes}_{d_{2}} K=$ $H \widehat{\otimes}_{g_{2}} K=H \otimes_{2} K$ whenever $H$ and $K$ are Hilbert spaces (here $H \otimes_{2} K$ is the usual Hilbertian tensor product). If we interpolate between $d_{2}$ and $g_{2}$ then we can verify that we end up with a symmetric tensor norm $\alpha$ such that $E \widehat{\otimes}_{\alpha} F$ is reflexive when $E$ and $F$ are, and such that $H \widehat{\otimes}_{\alpha} K=H \otimes_{2} K$ for Hilbert spaces $H$ and $K$.

Let $\mathcal{A}$ be a Banach algebra such that there is a reflexive Banach space $E$ and an isometric representation $\pi_{\mathcal{A}}: \mathcal{A} \rightarrow \mathcal{B}(E)$ such that $\pi_{\mathcal{A}}^{\prime} \kappa_{E^{\prime} \widehat{\otimes} E}$ takes the unit ball of $E^{\prime} \widehat{\otimes} E$ onto the unit ball of $\operatorname{WAP}\left(\mathcal{A}^{\prime}\right)$. From our previous work, this is equivalent to $\operatorname{WAP}\left(\mathcal{A}^{\prime}\right)$ being norming for $\mathcal{A}$. Suppose that $\mathcal{B}$ is similar, with $\pi_{\mathcal{B}}: \mathcal{B} \rightarrow \mathcal{B}(F)$, say. Now let $\alpha$ be some quasi-uniform crossnorm satisfying the conclusions of Proposition 8.9. Then $\pi=\pi_{\mathcal{A}} \otimes \pi_{\mathcal{B}}$ : $\mathcal{A} \otimes \mathcal{B} \rightarrow \mathcal{B}\left(E \widehat{\otimes}_{\alpha} F\right)$, defined by

$$
\pi(a \otimes b)(x \otimes y)=\pi_{\mathcal{A}}(a)(x) \otimes \pi_{\mathcal{B}}(b)(y) \quad(a \in \mathcal{A}, b \in \mathcal{B}, x \in E, y \in F),
$$

is a representation. Use this to induce a norm $\|\cdot\|_{\pi, \alpha}$ on $\mathcal{A} \otimes \mathcal{B}$, and denote the completion by $\mathcal{A} \widehat{\otimes}_{\pi, \alpha} \mathcal{B}$.

Proposition 8.11. With notation as above, $\|\cdot\|_{\pi, \alpha}$ is a WAP-crossnorm on $\mathcal{A} \otimes \mathcal{B}$.

Proof. By assumption, $\|a \otimes b\|_{\pi, \alpha}=\left\|\pi_{\mathcal{A}}(a)\right\|\left\|\pi_{\mathcal{B}}(b)\right\|=\|a\|\|b\|$ for $a \in \mathcal{A}$ and $b \in \mathcal{B}$, so the triangle inequality implies that $\|\cdot\|_{\pi, \alpha} \leq \pi(\cdot)$. Let $\lambda_{\mathcal{A}} \in \mathcal{A}^{\prime}$ and $\lambda_{\mathcal{B}} \in \mathcal{B}^{\prime}$ be such that $\left\|\lambda_{\mathcal{A}}\right\|=\left\|\lambda_{\mathcal{B}}\right\|=1$. As $\operatorname{WAP}\left(\mathcal{A}^{\prime}\right)$ is norming for $\mathcal{A}$, the unit ball of $\operatorname{WAP}\left(\mathcal{A}^{\prime}\right)$ is weak ${ }^{*}$-dense in the unit ball of $\mathcal{A}^{\prime}$, and so there exists a net $\left(\mu_{\alpha}^{\mathcal{A}}\right)$ in $\operatorname{WAP}\left(\mathcal{A}^{\prime}\right)$ such that $\left\|\mu_{\alpha}^{\mathcal{A}}\right\| \leq\left\|\lambda_{\mathcal{A}}\right\|$ for each $\alpha$, and $\lim _{\alpha}\left\langle\mu_{\alpha}^{\mathcal{A}}, a\right\rangle=\left\langle\lambda_{\mathcal{A}}, a\right\rangle$ for $a \in \mathcal{A}$. By the assumption on $\pi_{\mathcal{A}}$, for each $\alpha$, we can find $\sigma_{\alpha}^{\mathcal{A}} \in E^{\prime} \widehat{\otimes} E$ with $\left\|\sigma_{\alpha}^{\mathcal{A}}\right\|=\left\|\mu_{\alpha}^{\mathcal{A}}\right\|$ and $\pi_{\mathcal{A}^{\prime}}^{\prime} \kappa_{E^{\prime} \widehat{\otimes} E}\left(\sigma_{\alpha}^{\mathcal{A}}\right)=\mu_{\alpha}^{\mathcal{A}}$. Similarly, we can find $\left(\sigma_{\beta}^{\mathcal{B}}\right) \subseteq F^{\prime} \widehat{\otimes} F$ for $\lambda_{\mathcal{B}}$. Then, for $\tau=\sum_{k=1}^{n} a_{k} \otimes b_{k} \in \mathcal{A} \otimes \mathcal{B}$,

$$
\begin{aligned}
\left\langle\lambda_{\mathcal{A}} \otimes \lambda_{\mathcal{B}}, \tau\right\rangle & =\sum_{k=1}^{n} \lim _{\alpha}\left\langle\mu_{\alpha}^{\mathcal{A}}, a_{k}\right\rangle \lim _{\beta}\left\langle\mu_{\beta}^{\mathcal{B}}, b_{k}\right\rangle \\
& =\sum_{k=1}^{n} \lim _{\alpha}\left\langle\pi_{\mathcal{A}}\left(a_{k}\right), \sigma_{\alpha}^{\mathcal{A}}\right\rangle \lim _{\beta}\left\langle\pi_{\mathcal{B}}\left(b_{k}\right), \sigma_{\beta}^{\mathcal{B}}\right\rangle \\
& =\lim _{\alpha} \lim _{\beta}\left\langle\pi(\tau), \sigma_{\alpha}^{\mathcal{A}} \otimes \sigma_{\beta}^{\mathcal{B}}\right\rangle .
\end{aligned}
$$


By taking the supremum over $\left\|\lambda_{\mathcal{A}}\right\|=\left\|\lambda_{\mathcal{B}}\right\|=1$, we consequently conclude that $\epsilon(\cdot) \leq\|\cdot\|_{\pi, \alpha}$. Thus $\|\cdot\|_{\pi, \alpha}$ is a reasonable crossnorm, and so clearly it is an algebra crossnorm.

Now let $G=E \widehat{\otimes}_{\alpha} F$ and $\mathcal{C}=\mathcal{A} \widehat{\otimes}_{\pi, \alpha} \mathcal{B}$. We may treat $\pi$ as an isometry from $\mathcal{C}$ into $\mathcal{B}(G)$, and so $\pi^{\prime} \kappa_{G^{\prime} \widehat{\otimes} G}$ is a norm-decreasing map from $G^{\prime} \widehat{\otimes} G$ to $\mathcal{C}^{\prime}$. Define

$X=\left\{\pi^{\prime} \kappa_{G^{\prime} \widehat{\otimes} G}\left(\left(\mu_{E} \otimes \mu_{F}\right) \otimes\left(x_{E} \otimes x_{F}\right)\right): \mu_{E} \in E^{\prime}, \mu_{F} \in F^{\prime}, x_{E} \in E, x_{F} \in F\right\}$, so that clearly $X \subseteq \operatorname{WAP}\left(\mathcal{C}^{\prime}\right)$. For $\mu_{E} \in E^{\prime}, \mu_{F} \in F^{\prime}, x_{E} \in E$ and $x_{F} \in F$, we have, for $a \in \mathcal{A}$ and $b \in \mathcal{B}$,

$$
\begin{aligned}
\left\langle\pi^{\prime} \kappa_{G^{\prime} \widehat{\otimes} G}\left(\left(\mu_{E} \otimes \mu_{F}\right) \otimes\left(x_{E} \otimes x_{F}\right)\right)\right. & , a \otimes b\rangle \\
& =\left\langle\pi_{\mathcal{A}}^{\prime}\left(\mu_{E} \otimes x_{E}\right), a\right\rangle\left\langle\pi_{\mathcal{B}}^{\prime}\left(\mu_{F} \otimes x_{F}\right), b\right\rangle .
\end{aligned}
$$

Hence, by the assumptions on $\pi_{\mathcal{A}}$ and $\pi_{\mathcal{B}}$, the linear span of $X$ is dense in $\operatorname{WAP}\left(\mathcal{A}^{\prime}\right) \otimes \operatorname{WAP}\left(\mathcal{B}^{\prime}\right)$, and so $\operatorname{WAP}\left(\mathcal{A}^{\prime}\right) \otimes \operatorname{WAP}\left(\mathcal{B}^{\prime}\right) \subseteq \operatorname{WAP}\left(\mathcal{C}^{\prime}\right)$, as required. Finally, almost by definition, the linear span of $X$ is norming for $\pi(\mathcal{A} \otimes \mathcal{B})$, and so $\|\cdot\|_{\pi, \alpha}$ is a WAP-crossnorm.

Conversely, suppose that $\beta$ is some WAP-crossnorm on $\mathcal{A} \otimes \mathcal{B}$, so that there exists a reflexive Banach space $G$ and an isometric representation $\pi$ : $\mathcal{A} \widehat{\otimes}_{\beta} \mathcal{B} \rightarrow \mathcal{B}(G)$. However, it need not be the case that $G=E \widehat{\otimes}_{\alpha} F$ for some Banach spaces $E$ and $F$, and some uniform crossnorm $\alpha$, with $\pi=\pi_{\mathcal{A}} \otimes \pi_{\mathcal{B}}$ for suitable $\pi_{\mathcal{A}}: \mathcal{A} \rightarrow \mathcal{B}(E)$ and $\pi_{\mathcal{B}}: \mathcal{A} \rightarrow \mathcal{B}(F)$.

This mirrors the behaviour of $\mathrm{C}^{*}$-algebras. Recall that the minimal $\mathrm{C}^{*}$ tensor product of two $\mathrm{C}^{*}$-algebras $\mathcal{A}$ and $\mathcal{B}$ is that defined by taking faithful *-representations of $\mathcal{A}$ and $\mathcal{B}$ on Hilbert spaces $H$ and $K$, respectively, and letting $\mathcal{A} \otimes_{\min } \mathcal{B}$ be the closure of $\mathcal{A} \otimes \mathcal{B}$ in $\mathcal{B}\left(E \otimes_{2} F\right)$. It turns out that this is independent of the $*$-representations taken (as long as they are faithful). The maximal $\mathrm{C}^{*}$-tensor product is that defined by taking the supremum over any $*$-representation of $\mathcal{A} \otimes \mathcal{B}$. As indicated by their names, the minimal and maximal $\mathrm{C}^{*}$-tensor norms are indeed the smallest and greatest norms on $\mathcal{A} \otimes \mathcal{B}$ which satisfy the $\mathrm{C}^{*}$-condition $\left\|\tau^{*} \tau\right\|=\|\tau\|^{2}$ for $\tau \in \mathcal{A} \otimes \mathcal{B}$. Then a $\mathrm{C}^{*}$-algebra $\mathcal{A}$ is nuclear if $\mathcal{A} \otimes_{\min } \mathcal{B}=\mathcal{A} \otimes_{\max } \mathcal{B}$ for all $\mathrm{C}^{*}$-algebras $\mathcal{B}$. This is actually equivalent to $\mathcal{A}$ being amenable (see [29]).

As $\mathrm{C}^{*}$-algebras are always Arens regular, we see that a $\mathrm{C}^{*}$-tensor norm $\alpha$ on $\mathcal{A} \otimes \mathcal{B}$ is a WAP-crossnorm if and only if $\mathcal{A}^{\prime} \otimes \mathcal{B}^{\prime}$ is norming for $\mathcal{A} \widehat{\otimes}_{\alpha} \mathcal{B}$. However, this is always true for $\mathcal{A}^{\prime} \otimes_{\min } \mathcal{B}^{\prime}$, essentially for the same reasons as in the proof of Proposition 8.11. By [32, Proposition 4.10], the norm induced on $\mathcal{A}^{\prime} \otimes \mathcal{B}^{\prime}$ by $\mathcal{A} \otimes_{\max } \mathcal{B}$ always agrees with that induced by $\mathcal{A} \otimes_{\min } \mathcal{B}$, so if $\alpha$ is a $\mathrm{C}^{*}$-tensor norm, then as min $\leq \alpha \leq \max$, we see that $\mathcal{A}^{\prime} \widehat{\otimes}_{\alpha^{\mathrm{s}}} \mathcal{B}^{\prime}=\mathcal{A}^{\prime} \widehat{\otimes}_{\mathrm{min}^{\mathrm{s}}} \mathcal{B}^{\prime}$. Hence $\alpha$ is a WAP-crossnorm, as we might hope.

We may be tempted to define the minimal and maximal WAP-crossnorms in a similar fashion, given that we know that at least one WAP-crossnorm 
must exist. However, it is rather unclear if such minimal and maximal norms exist.

Proposition 8.12. Let $\mathcal{A}$ and $\mathcal{B}$ be Banach algebras. There exists an algebra crossnorm $\max$ on $\mathcal{A} \otimes \mathcal{B}$ such that if $\alpha$ is any WAP-crossnorm on $\mathcal{A} \otimes \mathcal{B}$, then $\alpha \leq \max$, and such that $\mathcal{A} \widehat{\otimes}_{\max } \mathcal{B}$ is a WAP-algebra.

Proof. Define $\max$ on $\mathcal{A} \otimes \mathcal{B}$ by $\max (\tau)=\sup \{\|\pi(\tau)\|\}$, the supremum being taken over all algebra homomorphisms $\pi: \mathcal{A} \otimes \mathcal{B} \rightarrow \mathcal{B}(E)$, where $E$ is a reflexive Banach space, and $\|\pi(a \otimes b)\|=\|a\|\|b\|$ for $a \in \mathcal{A}$ and $b \in \mathcal{B}$. Let $\|\cdot\|_{\pi, \alpha}$ be a WAP-crossnorm given by Proposition 8.11 , so that $\epsilon \leq\|\cdot\|_{\pi, \alpha} \leq \max$. As $\max (a \otimes b)=\|a\|\|b\|$ for $a \in \mathcal{A}$ and $b \in \mathcal{B}$, we see that $\max \leq \pi$, so that max is a reasonable crossnorm. Clearly then max is an algebra crossnorm.

Let $\alpha$ be some WAP-crossnorm on $\mathcal{A} \otimes \mathcal{B}$, so we can find a representation $\pi: \mathcal{A} \widehat{\otimes}_{\alpha} \mathcal{B} \rightarrow \mathcal{B}(E)$ for some reflexive Banach space $E$, such that $\pi^{\prime}$ takes the unit ball of $E^{\prime} \widehat{\otimes} E$ onto the unit ball of $\operatorname{WAP}\left(\left(\mathcal{A} \widehat{\otimes}_{\alpha} \mathcal{B}\right)^{\prime}\right)$. As $\operatorname{WAP}\left(\mathcal{A}^{\prime}\right) \otimes$ $\mathrm{WAP}\left(\mathcal{B}^{\prime}\right) \subseteq \operatorname{WAP}\left(\left(\mathcal{A} \widehat{\otimes}_{\alpha} \mathcal{B}\right)^{\prime}\right)$ is norming for $\mathcal{A} \widehat{\otimes}_{\alpha} \mathcal{B}$, we see that $\pi$ is an isometry. Consequently, $\alpha \leq \max$.

For each $\tau \in \mathcal{A} \otimes \mathcal{B}$ and each $\varepsilon>0$, let $\pi_{\tau, \varepsilon}: \mathcal{A} \otimes \mathcal{B} \rightarrow \mathcal{B}\left(E_{\tau, \varepsilon}\right)$ be some representation on a reflexive Banach space such that $\left\|\pi_{\tau, \varepsilon}(\tau)\right\|>\max (\tau)-\varepsilon$. Let $F=\ell^{2}\left(\bigoplus_{\tau, \varepsilon} E_{\tau, \varepsilon}\right)$, so that $\pi=\bigoplus \pi_{\tau, \varepsilon}$ is a representation of $\mathcal{A} \otimes \mathcal{B}$ on $\mathcal{B}(F)$. Clearly then, for $\tau \in \mathcal{A} \otimes \mathcal{B},\|\pi(\tau)\|=\sup \left\|\pi_{\tau, \varepsilon}(\tau)\right\|=\max (\tau)$, and so $\pi$ extends to an isometric representation $\pi: \mathcal{A} \widehat{\otimes}_{\max } \mathcal{B} \rightarrow \mathcal{B}(F)$.

In general, however, we see no way to show that this norm is a WAPcrossnorm.

8.2. Dual Banach algebras. Recall that for two $W^{*}$-algebras $\left(\mathcal{A}, \mathcal{A}_{*}\right)$ and $\left(\mathcal{B}, \mathcal{B}_{*}\right)$, we let $X=\mathcal{A} \otimes \min \mathcal{B}$, and then regard $\mathcal{A}_{*} \otimes \mathcal{B}_{*}$ as a subspace of $X^{\prime}$, with closure $\mathcal{A}_{*} \bar{\otimes} \mathcal{B}_{*}$. Then $\mathcal{A} \bar{\otimes} \mathcal{B}$, the $W^{*}$-tensor product of $\mathcal{A}$ and $\mathcal{B}$, is the dual of $\mathcal{A}_{*} \bar{\otimes} \mathcal{B}_{*}$. It may be checked that $\mathcal{A} \otimes \mathcal{B}$ becomes a weak ${ }^{*}$-dense subalgebra of $\mathcal{A} \bar{\otimes} \mathcal{B}$. For example, when $H$ and $K$ are Hilbert spaces, we have $\mathcal{B}(H) \bar{\otimes}(K)=\mathcal{B}\left(H \otimes_{2} K\right)$; in particular, $\mathcal{B}(H) \otimes \mathcal{B}(K)$ need not be norm-dense in $\mathcal{B}(H) \bar{\otimes} \mathcal{B}(K)$ (see [19, Exercise 11.5.7]).

Let $\left(\mathcal{A}, \mathcal{A}_{*}\right)$ and $\left(\mathcal{B}, \mathcal{B}_{*}\right)$ be dual Banach algebras, and let $\beta$ be a WAPcrossnorm on $\mathcal{A} \otimes \mathcal{B}$. By assumption, $\operatorname{WAP}\left(\mathcal{A}^{\prime}\right) \widehat{\otimes}_{\beta^{s}} \operatorname{WAP}\left(\mathcal{B}^{\prime}\right)$ is norming for $\mathcal{A} \widehat{\otimes}_{\beta} \mathcal{B}$ and maps into $\operatorname{WAP}\left(\left(\mathcal{A} \widehat{\otimes}_{\beta} \mathcal{B}\right)^{\prime}\right)$. Hence we see that $X=\mathcal{A}_{*} \widehat{\otimes}_{\beta^{\mathrm{s}}} \mathcal{B}_{*}$ is a closed $\mathcal{A} \widehat{\otimes}_{\beta} \mathcal{B}$-submodule of $\operatorname{WAP}\left(\left(\mathcal{A} \widehat{\otimes}_{\beta} \mathcal{B}\right)^{\prime}\right)$. Thus, by Proposition 2.4, $\left(X^{\prime}, X\right)$ becomes a dual Banach algebra. Notice that the norm on $X=$ $\mathcal{A}_{*} \widehat{\otimes}_{\beta^{\mathrm{s}}} \mathcal{B}_{*}$ is given by

$$
\|\tau\|_{X}=\sup \{|\langle\tau, u\rangle|: u \in \mathcal{A} \otimes \mathcal{B}, \beta(u) \leq 1\} \quad(\tau \in X)
$$

It is hence immediate that $\mathcal{A} \otimes \mathcal{B}$ is weak*-dense in $X^{\prime}$, so we may regard $X^{\prime}$ as a dual Banach algebra tensor product of $\mathcal{A}$ and $\mathcal{B}$, and denote $X^{\prime}$ by 
$\mathcal{A} \bar{\otimes}_{\beta} \mathcal{B}$. When $\mathcal{A}_{*} \widehat{\otimes}_{\beta^{\mathrm{s}}} \mathcal{B}_{*}$ is norming for $\mathcal{A} \widehat{\otimes}_{\beta} \mathcal{B}$, we see that $\mathcal{A} \widehat{\otimes}_{\beta} \mathcal{B}$ is even a closed subspace of $\mathcal{A} \bar{\otimes}_{\beta} \mathcal{B}$.

EXAMPLE 8.13. Let $G$ and $H$ be locally compact groups, so that $M(G)$ is a dual Banach algebra with predual $C_{0}(G)$, and similarly for $H$ (see Example 8.6). By Proposition 8.7, $\operatorname{WAP}\left(M(G)^{\prime}\right) \otimes \mathrm{WAP}\left(M(H)^{\prime}\right)$ maps into $\mathrm{WAP}\left((M(G) \widehat{\otimes} M(H))^{\prime}\right)$. As the map $M(G) \widehat{\otimes} M(H) \rightarrow\left(C_{0}(G) \check{\otimes} C_{0}(H)\right)^{\prime}$ is an isometry, we see that

$$
C_{0}(G) \otimes C_{0}(H) \subseteq C_{0}(G) \check{\otimes} C_{0}(G) \subseteq(M(G) \widehat{\otimes} M(H))^{\prime}=\mathcal{B}\left(M(G), M(H)^{\prime}\right)
$$

is norming for $M(G) \widehat{\otimes} M(H)$. Thus $\pi$ is a WAP-crossnorm on $M(G) \otimes M(H)$.

We may hence form $M(G) \bar{\otimes}_{\pi} M(H)$. By definition, it is the weak ${ }^{*}$-closure of $M(G) \otimes M(H)$ in the dual of $C_{0}(G) \check{\otimes} C_{0}(H)$, that is, in $M(G \times H)$. As explained in Example 8.6, $M(G) \otimes M(H)$ is certainly weak ${ }^{*}$-dense in $M(G \times H)$, and so $M(G) \bar{\otimes}_{\pi} M(H)=M(G \times H)$ as we might hope.

Given dual Banach algebras $\left(\mathcal{A}, \mathcal{A}_{*}\right)$ and $\left(\mathcal{B}, \mathcal{B}_{*}\right)$, we can find weak*weak*-continuous representations $\pi_{\mathcal{A}}: \mathcal{A} \rightarrow \mathcal{B}(E)$ and $\pi_{\mathcal{B}}: \mathcal{B} \rightarrow \mathcal{B}(F)$. We may hence define the WAP-crossnorm $\beta:=\|\cdot\|_{\pi, \alpha}$ as in Proposition 8.11, leading to $\mathcal{A} \widehat{\otimes}_{\pi, \alpha} \mathcal{B}$ and hence $\mathcal{A} \bar{\otimes}_{\beta} \mathcal{B}$. Alternatively, we may simply define $\mathcal{A} \bar{\otimes}_{\pi, \alpha} \mathcal{B}$ to be the weak*-closure of $\pi_{\mathcal{A}}(\mathcal{A}) \otimes \pi_{\mathcal{B}}(\mathcal{B})$ in $\mathcal{B}\left(E \widehat{\otimes}_{\alpha} F\right)$.

Proposition 8.14. With notation as above, there is a natural normdecreasing map from $\mathcal{A}_{*} \widehat{\otimes}_{\beta^{\mathrm{s}}} \mathcal{B}_{*}$ to $\left(\mathcal{A} \bar{\otimes}_{\pi, \alpha} \mathcal{B}\right)_{*}$ which has dense range. If $\mathcal{A}_{*} \widehat{\otimes}_{\beta^{\mathrm{s}}} \mathcal{B}_{*}$ is norming for $\mathcal{A} \widehat{\otimes}_{\beta} \mathcal{B}$, then this map is an isometry.

Proof. We identify $\mathcal{A}$ with its weak*-closed image under $\pi_{\mathcal{A}}: \mathcal{A} \rightarrow \mathcal{B}(E)$, and hence we identify $\mathcal{A}_{*}$ with a quotient of $E^{\prime} \widehat{\otimes} E$, namely $E^{\prime} \widehat{\otimes} E /{ }^{\perp} \mathcal{A}$, and similarly for $\mathcal{B}$. Then $\mathcal{A} \widehat{\otimes}_{\beta} \mathcal{B}$ is the closure of $\mathcal{A} \otimes \mathcal{B}$ in $\mathcal{B}\left(E \widehat{\otimes}_{\alpha} F\right)$. We deduce that $\left(\mathcal{A} \bar{\otimes}_{\pi, \alpha} \mathcal{B}\right)_{*}=\mathcal{B}\left(E \widehat{\otimes}_{\alpha} F\right)_{*} /{ }^{\perp}(\mathcal{A} \otimes \mathcal{B})$ where $\mathcal{B}\left(E \widehat{\otimes}_{\alpha} F\right)_{*}=$ $\left(E^{\prime} \widehat{\otimes}_{\alpha^{s}} F^{\prime}\right) \widehat{\otimes}\left(E \widehat{\otimes}_{\alpha} F\right)$.

We define a natural map $\theta: \mathcal{A}_{*} \otimes \mathcal{B}_{*} \rightarrow\left(\mathcal{A} \bar{\otimes}_{\pi, \alpha} \mathcal{B}\right)_{*}$ as follows. For $\tau \in \mathcal{A}_{*}, \sigma \in \mathcal{B}_{*}$ and $\varepsilon>0$, we have representations

$\tau=\sum_{n=1}^{\infty} x_{n}^{E} \otimes \mu_{n}^{E}+{ }^{\perp} \mathcal{A} \in E^{\prime} \widehat{\otimes} E /{ }^{\perp} \mathcal{A}, \quad \sigma=\sum_{n=1}^{\infty} x_{n}^{F} \otimes \mu_{n}^{F}+{ }^{\perp} \mathcal{B} \in F^{\prime} \widehat{\otimes} F /{ }^{\perp} \mathcal{B}$, where $\sum_{n=1}^{\infty}\left\|x_{n}^{E}\right\|\left\|\mu_{n}^{E}\right\|<\|\tau\|+\varepsilon$ and $\sum_{n=1}^{\infty}\left\|x_{n}^{F}\right\|\left\|\mu_{n}^{F}\right\|<\|\sigma\|+\varepsilon$. Then let $\theta(\tau \otimes \sigma)=u=\sum_{n=1}^{\infty} \sum_{m=1}^{\infty}\left(x_{n}^{E} \otimes x_{m}^{F}\right) \otimes\left(\mu_{n}^{E} \otimes \mu_{m}^{F}\right)+{ }^{\perp}(\mathcal{A} \otimes \mathcal{B}) \in\left(\mathcal{A} \bar{\otimes}_{\pi, \alpha} \mathcal{B}\right)_{*}$, so that $\|u\| \leq \sum_{n=1}^{\infty} \sum_{m=1}^{\infty}\left\|x_{n}^{E}\right\|\left\|x_{m}^{F}\right\|\left\|\mu_{n}^{E}\right\|\left\|\mu_{m}^{F}\right\|<(\|\sigma\|+\varepsilon)(\|\tau\|+\varepsilon)$. For $a \in \mathcal{A}$ and $b \in \mathcal{B}$, we see that $\langle a \otimes b, u\rangle=\sum_{n=1}^{\infty} \sum_{m=1}^{\infty}\left\langle a, x_{n}^{E} \otimes \mu_{n}^{E}\right\rangle\left\langle b, x_{m}^{F} \otimes \mu_{m}^{F}\right\rangle=\langle a, \tau\rangle\langle b, \sigma\rangle=\langle a \otimes b, \tau \otimes \sigma\rangle$. 
In particular, $u$ does not depend upon the choice of representatives for $\tau$ and $\sigma$, and so $\theta$ is well-defined. Notice also that for $\psi \in \mathcal{A}_{*} \otimes \mathcal{B}_{*}$,

$$
\begin{aligned}
\beta^{\mathrm{s}}(\psi) & =\sup \{|\langle\psi, v\rangle|: v \in \mathcal{A} \otimes \mathcal{B}, \beta(v) \leq 1\} \\
& =\sup \left\{|\langle\theta(\psi), v\rangle|: v \in \mathcal{A} \otimes \mathcal{B} \subseteq \mathcal{B}\left(E \widehat{\otimes}_{\alpha} F\right),\|v\| \leq 1\right\} \leq\|\theta(\psi)\|,
\end{aligned}
$$

so that $\theta$ extends to a norm-decreasing map $\mathcal{A}_{*} \widehat{\otimes}_{\beta^{\mathrm{s}}} \mathcal{B}_{*} \rightarrow\left(\mathcal{A} \bar{\otimes}_{\pi, \alpha} \mathcal{B}\right)_{*}$. Notice that if we knew that the unit ball of $\mathcal{A} \otimes \mathcal{B}$ were weak ${ }^{*}$-dense in the unit ball of $\mathcal{A} \bar{\otimes}_{\pi, \alpha} \mathcal{B}$ (that is, $\mathcal{A}_{*} \widehat{\otimes}_{\beta^{\mathrm{s}}} \mathcal{B}_{*}$ were norming for $\mathcal{A} \widehat{\otimes}_{\beta} \mathcal{B}$ ), then $\theta$ would even be an isometry. This is the case for von Neumann algebras, for example, by the Kaplansky density theorem ([32, Section II, Theorem 4.8]).

We shall now show that $\mathcal{A}_{*} \otimes \mathcal{B}_{*}$ is dense in $\left(\mathcal{A} \bar{\otimes}_{\pi, \alpha} \mathcal{B}\right)_{*}$, which will complete the proof. Let $\tau \in\left(\mathcal{A} \bar{\otimes}_{\pi, \alpha} \mathcal{B}\right)_{*}$, and pick a representation

$$
\tau=\sum_{n=1}^{\infty} u_{n} \otimes v_{n}+{ }^{\perp}(\mathcal{A} \otimes \mathcal{B})
$$

with $\left(u_{n}\right) \subseteq E^{\prime} \widehat{\otimes}_{\alpha^{\mathrm{s}}} F^{\prime}$ and $\left(v_{n}\right) \subseteq E \widehat{\otimes}_{\alpha} F$ satisfying $\sum_{n=1}^{\infty} \alpha^{\mathrm{s}}\left(u_{n}\right) \alpha\left(v_{n}\right)$ $<\infty$. By approximation, we may actually suppose that $\left(u_{n}\right) \subseteq E^{\prime} \otimes F^{\prime}$ and $\left(v_{n}\right) \subseteq E \otimes F$. We can then find representations

$$
u_{n}=\sum_{k=1}^{\infty} \phi_{k}^{(n)} \otimes \psi_{k}^{(n)}, \quad v_{n}=\sum_{k=1}^{\infty} x_{k}^{(n)} \otimes y_{k}^{(n)},
$$

where for each $n$, eventually $\phi_{k}^{(n)}=0$, and so forth. For each $n$, define

$$
\mu_{n}=\sum_{k=1}^{\infty} \phi_{k}^{(n)} \otimes x_{k}^{(n)}+{ }^{\perp} \mathcal{A} \in \mathcal{A}_{*}, \quad \lambda_{n}=\sum_{k=1}^{\infty} \psi_{k}^{(n)} \otimes y_{k}^{(n)}+{ }^{\perp} \mathcal{B} \in \mathcal{B}_{*},
$$

noticing that each of these is a finite sum. For $a \in \mathcal{A}$ and $b \in \mathcal{B}$, we see that

$$
\left\langle a \otimes b, \mu_{n} \otimes \lambda_{n}\right\rangle=\sum_{k=1}^{\infty}\left\langle a, \phi_{k}^{(n)} \otimes x_{k}^{(n)}\right\rangle\left\langle b, \psi_{k}^{(n)} \otimes y_{k}^{(n)}\right\rangle=\left\langle a \otimes b, u_{n} \otimes v_{n}\right\rangle
$$

for $n \geq 1$. Consequently, for $c \in \mathcal{A} \bar{\otimes}_{\pi, \alpha} \mathcal{B}$ and $N \geq 1$, as $\mathcal{A} \otimes \mathcal{B}$ is weak*-dense in $\mathcal{A} \bar{\otimes}_{\pi, \alpha} \mathcal{B}$

$$
\left|\langle c, \tau\rangle-\sum_{n=1}^{N}\left\langle c, \mu_{n} \otimes \lambda_{n}\right\rangle\right|=\left|\sum_{n=N+1}^{\infty}\left\langle c, u_{n} \otimes v_{n}\right\rangle\right| \leq\|c\| \sum_{n=N+1}^{\infty} \alpha^{\mathrm{s}}\left(u_{n}\right) \alpha\left(v_{n}\right) .
$$

Thus $\mathcal{A}_{*} \otimes \mathcal{B}_{*}$ is indeed dense in $\left(\mathcal{A} \bar{\otimes}_{\pi, \alpha} \mathcal{B}\right)_{*}$.

As indicated, the lack of a generalisation of the Kaplansky density theorem shows that in general $\mathcal{A} \bar{\otimes}_{\beta} \mathcal{B}$ and $\mathcal{A} \bar{\otimes}_{\pi, \alpha} \mathcal{B}$ are different. The following provides an example of a general Banach algebra in which the theory works well. 
Proposition 8.15. Let $E$ and $F$ be reflexive Banach spaces, and let $\alpha$ be a tensor norm on $E \otimes F$ satisfying the conclusions of Proposition 8.9. Form the tensor products $\mathcal{B}(E) \bar{\otimes}_{\beta} \mathcal{B}(F)$ and $\mathcal{B}(E) \bar{\otimes}_{\pi, \alpha} \mathcal{B}(F)$ by using the trivial representations of $\mathcal{B}(E)$ on itself, and the same for $\mathcal{B}(F)$. Then these tensor products agree with $\mathcal{B}\left(E \widehat{\otimes}_{\alpha} F\right)$.

Proof. By Proposition 8.14, $\mathcal{B}(E)_{*} \otimes \mathcal{B}(F)_{*}$ is dense in the predual of $\mathcal{B}(E) \bar{\otimes}_{\pi, \alpha} \mathcal{B}(F)$. Furthermore, $E^{\prime} \otimes E \otimes F^{\prime} \otimes F$ is dense in both $\mathcal{B}(E)_{*} \otimes \mathcal{B}(F)_{*}$ and $\mathcal{B}\left(E \widehat{\otimes}_{\alpha} F\right)_{*}$. So if our natural map is an isometry, that is, $\mathcal{B}(E)_{*} \widehat{\otimes}_{\beta} \mathcal{B}(F)_{*}$ is norming for $\mathcal{B}(E) \widehat{\otimes}_{\beta} \mathcal{B}(F)$, then the proof is complete.

If we identify $\mathcal{B}(E) \otimes \mathcal{B}(F)$ as a subalgebra of $\mathcal{B}\left(E \widehat{\otimes}_{\alpha} F\right)$ then $\beta$ agrees with the operator norm. Let $u \in \mathcal{B}(E) \otimes \mathcal{B}(F)$ and $\varepsilon>0$, so we may find $\sigma \in$ $E^{\prime} \otimes F^{\prime}$ and $\tau \in E \otimes F$ with $\alpha^{\mathrm{s}}(\sigma) \leq 1, \alpha(\tau) \leq 1$ and $|\langle\sigma, u(\tau)\rangle|>\beta(u)-\varepsilon$. Let

$$
\sigma=\sum_{i=1}^{n} \mu_{i} \otimes \lambda_{i}, \quad \tau=\sum_{j=1}^{m} x_{j} \otimes y_{j}
$$

and define

$$
v=\sum_{i=1}^{n} \sum_{j=1}^{m}\left(\mu_{i} \otimes x_{j}\right) \otimes\left(\lambda_{i} \otimes y_{j}\right) \in\left(E^{\prime} \otimes E\right) \otimes\left(F^{\prime} \otimes F\right) \subseteq \mathcal{B}(E)_{*} \otimes \mathcal{B}(F)_{*} .
$$

A simple calculation shows that $\langle w, v\rangle=\langle\sigma, w(\tau)\rangle$ for any $w \in \mathcal{B}(E) \otimes \mathcal{B}(F)$, so that

$$
\begin{aligned}
\beta^{\mathrm{S}}(v) & =\sup \{|\langle w, v\rangle|: w \in \mathcal{B}(E) \otimes \mathcal{B}(F), \beta(w) \leq 1\} \\
& =\sup \{|\langle\sigma, w(\tau)\rangle|:\|w\| \leq 1\} \leq\|\sigma\|\|\tau\| \leq 1 .
\end{aligned}
$$

It hence follows that the norm of $u$ as a member of the dual space of $\mathcal{B}(E)_{*} \widehat{\otimes}_{\beta^{\mathrm{s}}} \mathcal{B}(F)_{*}$ is at least $\beta(u)-\varepsilon$. The proof is complete, as $\varepsilon>0$ was arbitrary.

It would be nice if we could find a universal way to take the tensor product of two dual Banach algebras. For example, the projective tensor product of two Banach algebras always gives a Banach algebra (although it is not always the most natural norm to use, for example for $C(K)$ spaces). This problem is related to the fact that we cannot find maximal or minimal WAP-crossnorms.

Let $\mathcal{A}$ and $\mathcal{B}$ be WAP-algebras, and let $\alpha$ be a WAP-crossnorm on $\mathcal{A} \otimes \mathcal{B}$. It would be natural if there was some connection between the DBA enveloping algebra $\operatorname{WAP}\left(\left(\mathcal{A} \widehat{\otimes}_{\alpha} \mathcal{B}\right)^{\prime}\right)^{\prime}$ and the dual Banach algebra tensor product $\operatorname{WAP}\left(\mathcal{A}^{\prime}\right)^{\prime} \bar{\otimes}_{\alpha} \operatorname{WAP}\left(\mathcal{B}^{\prime}\right)^{\prime}$. However, this latter algebra is the dual of $\operatorname{WAP}\left(\mathcal{A}^{\prime}\right) \widehat{\otimes}_{\alpha^{s}} \operatorname{WAP}\left(\mathcal{B}^{\prime}\right)$, which is only a norming submodule of $\operatorname{WAP}\left(\left(\mathcal{A} \widehat{\otimes}_{\alpha} \mathcal{B}\right)^{\prime}\right)$. Hence, in general, $\operatorname{WAP}\left(\mathcal{A}^{\prime}\right)^{\prime} \bar{\otimes}_{\alpha} \operatorname{WAP}\left(\mathcal{B}^{\prime}\right)^{\prime}$ is only a quotient of $\operatorname{WAP}\left(\left(\mathcal{A} \widehat{\otimes}_{\alpha} \mathcal{B}\right)^{\prime}\right)^{\prime}$. Example 8.4 shows that this is true even for commutative $\mathrm{C}^{*}$-algebras. 


\subsection{Application to Connes-amenability}

Theorem 8.16. Let $\mathcal{A}$ and $\mathcal{B}$ be Connes-amenable dual Banach algebras, and let $\beta$ be a reasonable crossnorm on $\mathcal{A}_{*} \otimes \mathcal{B}_{*}$ which turns $\left(\mathcal{A}_{*} \widehat{\otimes}_{\beta} \mathcal{B}_{*}\right)^{\prime}=$ $\mathcal{A} \bar{\otimes} \mathcal{B}$ into a dual Banach algebra containing $\mathcal{A} \widehat{\otimes}_{\beta^{s}} \mathcal{B}$ as a weak $k^{*}$-dense Banach algebra (so that $\beta^{\mathrm{s}}$ is an algebra crossnorm on $\mathcal{A} \otimes \mathcal{B}$ ). Then $\mathcal{A} \bar{\otimes} \mathcal{B}$ is Connes-amenable.

Proof. Let $E$ be a reflexive Banach space, and let $\pi: \mathcal{A} \bar{\otimes} \mathcal{B} \rightarrow \mathcal{B}(E)$ be a weak ${ }^{*}$-continuous representation. As in the proof of Proposition 6.15, we claim that $\pi(\mathcal{A} \bar{\otimes} \mathcal{B})^{\mathrm{c}}=\pi(\mathcal{A} \otimes \mathcal{B})^{\mathrm{c}}$, which follows as $\pi$ is weak ${ }^{*}$-continuous and $\mathcal{A} \otimes \mathcal{B}$ is weak*-dense in $\mathcal{A} \otimes \mathcal{B}$. We wish to show that there is a quasiexpectation $\mathcal{Q}: \mathcal{B}(E) \rightarrow \pi(\mathcal{A} \otimes \mathcal{B})^{\mathrm{c}}$.

As $\mathcal{A}$ and $\mathcal{B}$ are Connes-amenable, they are unital, with units $e_{\mathcal{A}}$ and $e_{\mathcal{B}}$, say. We may define a homomorphism $\phi: \mathcal{A} \rightarrow \mathcal{A} \bar{\otimes} \mathcal{B}$ by $\phi(a)=a \otimes e_{\mathcal{B}}$ for $a \in \mathcal{A}$. Then, for $\tau=\sum_{k=1}^{n} \mu_{k} \otimes \lambda_{k} \in \mathcal{A}_{*} \otimes \mathcal{B}_{*}$, we see that

$$
\langle\phi(a), \tau\rangle=\left\langle a \otimes e_{\mathcal{B}}, \tau\right\rangle=\sum_{k=1}^{n}\left\langle a, \mu_{k}\right\rangle\left\langle e_{\mathcal{B}}, \lambda_{k}\right\rangle=\left\langle a, \phi_{*}(\tau)\right\rangle \quad(a \in \mathcal{A}),
$$

where $\phi_{*}(\tau)=\sum_{k=1}^{n}\left\langle e_{\mathcal{B}}, \lambda_{k}\right\rangle \mu_{k} \in \mathcal{A}_{*}$. Clearly $\phi_{*}$ is bounded (as $\beta$ is a reasonable crossnorm), so $\phi_{*}$ extends to $\mathcal{A}_{*} \widehat{\otimes}_{\beta} \mathcal{B}_{*}=(\mathcal{A} \bar{\otimes} \mathcal{B})_{*}$, and we see that $\phi_{*}^{\prime}=\phi$, so that $\phi$ is weak ${ }^{*}$-continuous. A similar remark holds for $\mathcal{B}$.

Consider the representation $\pi_{\mathcal{A}}: \mathcal{A} \rightarrow \mathcal{B}(E)$ given by $\pi_{\mathcal{A}}(a)=\pi\left(a \otimes e_{\mathcal{B}}\right)$. This is weak*-continuous by the preceding paragraph, so identify $\mathcal{A}$ with its image in $\mathcal{B}(E)$. As $\mathcal{A}$ is Connes-amenable, there is a quasi-expectation $\mathcal{Q}_{\mathcal{A}}$ : $\mathcal{B}(E) \rightarrow \mathcal{A}^{\mathrm{c}}$. Analogously, there exists a quasi-expectation $\mathcal{Q}_{\mathcal{B}}: \mathcal{B}(E) \rightarrow \mathcal{B}^{\mathrm{c}}$. Notice that $\mathcal{A} \subseteq \mathcal{B}^{\mathrm{c}}, \mathcal{B} \subseteq \mathcal{A}^{\mathrm{c}}$ and $(\mathcal{A} \otimes \mathcal{B})^{\mathrm{c}}=\mathcal{A}^{\mathrm{c}} \cap \mathcal{B}^{\mathrm{c}}$.

Let $\mathcal{Q}=\mathcal{Q}_{\mathcal{B}} \mathcal{Q}_{\mathcal{A}}$, so that $\mathcal{Q}$ is bounded, and $\mathcal{Q}(a)=a$ for each $a \in \mathcal{A}^{\mathrm{c}} \cap \mathcal{B}^{\mathrm{c}}$. Let $T \in \mathcal{B}(E)$, let $x=\mathcal{Q}_{\mathcal{A}}(T) \in \mathcal{A}^{\mathrm{c}}$, and let $b=\mathcal{Q}_{\mathcal{B}}(x)=\mathcal{Q}(T) \in \mathcal{B}^{\mathrm{c}}$. Let $a \in \mathcal{A}$, so that $a x=x a$, and as $\mathcal{A} \subseteq \mathcal{B}^{\mathrm{c}}$,

$$
\begin{aligned}
\mathcal{Q}_{\mathcal{B}}(a(b-x)) & =a \mathcal{Q}_{\mathcal{B}}(b-x)=0=\mathcal{Q}_{\mathcal{B}}(b-x) a \\
& =\mathcal{Q}_{\mathcal{B}}((b-x) a) .
\end{aligned}
$$

As $a b, b a \in \mathcal{B}^{\text {c }}$, we see that $a b=\mathcal{Q}_{\mathcal{B}}(a b)=\mathcal{Q}_{\mathcal{B}}(b a)=b a$, so we conclude that $b \in \mathcal{A}^{\mathrm{c}}$. Thus $\mathcal{Q}$ maps into $\mathcal{A}^{\mathrm{c}} \cap \mathcal{B}^{\mathrm{c}}$, and so we conclude that $\mathcal{Q}$ is a projection onto $\mathcal{A}^{\mathrm{c}} \cap \mathcal{B}^{\mathrm{c}}$. Now let $a, b \in \mathcal{A}^{\mathrm{c}} \cap \mathcal{B}^{\mathrm{c}}$, and let $T \in \mathcal{B}(E)$, so that

$$
\mathcal{Q}(a T b)=\mathcal{Q}_{\mathcal{B}} \mathcal{Q}_{\mathcal{A}}(a T b)=\mathcal{Q}_{\mathcal{B}}\left(a \mathcal{Q}_{\mathcal{A}}(T) b\right)=a \mathcal{Q}(T) b,
$$

and we conclude that $\mathcal{Q}$ is a quasi-expectation, as required.

Notice that this proof will also show that $\mathcal{C}$ is Connes-amenable whenever $\mathcal{C}$ is a dual Banach algebra containing $\mathcal{A} \otimes \mathcal{B}$ as a dense subalgebra, and is such that the map $\mathcal{A} \rightarrow \mathcal{C}, a \mapsto a \otimes e_{\mathcal{B}}$, is weak*-continuous (and similarly for $\mathcal{B})$. 
Corollary 8.17. Let $r, s \in(1, \infty)$, and let $\alpha$ be some quasi-uniform crossnorm on $\ell^{r} \otimes \ell^{s}$ such that $\ell^{r} \widehat{\otimes}_{\alpha} \ell^{s}$ is reflexive, and $\left(\ell^{r}\right)^{\prime} \otimes\left(\ell^{s}\right)^{\prime}$ is dense in $\left(\ell^{r} \widehat{\otimes}_{\alpha} \ell^{s}\right)^{\prime}$. Then $\mathcal{B}\left(\ell^{r} \widehat{\otimes}_{\alpha} \ell^{s}\right)$ is Connes-amenable.

Proof. By Proposition 8.15, we have $\mathcal{B}\left(\ell^{r} \widehat{\otimes}_{\alpha} \ell^{s}\right)=\mathcal{B}\left(\ell^{r}\right) \bar{\otimes}_{\alpha} \mathcal{B}\left(\ell^{s}\right)$. Then the theorem applies, as $\mathcal{B}\left(\ell^{r}\right)$ and $\mathcal{B}\left(\ell^{s}\right)$ are Connes-amenable, by results in [30].

This corollary is comparable to [17, Theorem 2.2], as a quasi-uniform tensor norm is tight in the sense of [17], and by [30], the amenability of $\mathcal{A}(E)$ is equivalent to the Connes-amenability of $\mathcal{B}(E)$, at least when $E$ is reflexive and has the approximation property.

It is interesting to note that our proof of Theorem 8.16 is rather more algebraic than an analogous von Neumann result (compare to [33, Chapter XV, Proposition 3.2]). Our approach is more in line with that of [29, Proposition 6.3.17].

Acknowledgements. The author would like to thank the anonymous reviewer for much helpful advice.

\section{References}

[1] R. Aharoni and P. D. Saphar, On the reflexivity of the space $\pi_{p}(E, F)$ of p-absolutely summing operators, $1 \leq p<+\infty$, Bull. London Math. Soc. 25 (1993), 362-368.

[2] W. G. Bade, The Banach space $C(S)$, Lecture Notes Ser. 26, Mat. Inst., Aarhus Univ., Aarhus, 1971.

[3] B. Beauzamy, Espaces d'Interpolation Réels: Topologie et Géométrie, Springer, Berlin, 1978.

[4] J. F. Berglund, H. D. Junghenn and P. Milnes, Analysis on Semigroups. Function Spaces, Compactifications, Representations, Wiley, New York, 1989.

[5] J. W. Bunce and W. L. Paschke, Quasi-expectations and amenable von Neumann algebras, Proc. Amer. Math. Soc. 71 (1978), 232-236.

[6] T. K. Carne, Tensor products and Banach algebras, J. London Math. Soc. 17 (1978), 480-488.

[7] G. Corach and J. E. Galé, On amenability and geometry of spaces of bounded representations, ibid. 59 (1999), 311-329.

[8] H. G. Dales, Banach Algebras and Automatic Continuity, Clarendon Press, Oxford, 2000 .

[9] H. G. Dales and A. T.-M. Lau, The second duals of Beurling algebras, Mem. Amer. Math. Soc. 177 (2005), no. 836.

[10] W. J. Davis, T. Figiel, W. B. Johnson and A. Pełczyński, Factoring weakly compact operators, J. Funct. Anal. 17 (1974), 311-327.

[11] M. Daws, Connes-amenability of bidual and weighted semigroup algebras, Math. Scand., to appear (see arXiv:math.FA/0508552).

[12] M. Daws and N. J. Laustsen, Involutions on algebras of operators on a Banach space, preprint.

[13] A. Defant and K. Floret, Tensor Norms and Operator Ideals, North-Holland, Amsterdam, 1993. 
[14] J. Diestel and J. J. Uhl, Jr., Vector Measures, Amer. Math. Soc., Providence, RI, 1977.

[15] G. Godefroy, Existence and uniqueness of isometric preduals: a survey, in: Banach Space Theory (Iowa City, IA, 1987), Contemp. Math. 85, Amer. Math. Soc., Providence, RI, 1989, 131-193.

[16] G. Godefroy and P. D. Saphar, Duality in spaces of operators and smooth norms on Banach spaces, Illinois J. Math. 32 (1988), 672-695.

[17] N. Grønbæk, B. E. Johnson and G. A. Willis, Amenability of Banach algebras of compact operators, Israel J. Math. 87 (1994), 289-324.

[18] P. R. Halmos, Measure Theory, Van Nostrand, New York, 1950.

[19] R. V. Kadison and J. R. Ringrose, Fundamentals of the Theory of Operator Algebras. Vol. II, Amer. Math. Soc., Providence, RI, 1997.

[20] S. Kaijser, On Banach modules. I, Math. Proc. Cambridge Philos. Soc. 90 (1981), $423-444$.

[21] A. T.-M. Lau and R. J. Loy, Banach algebras on compact right topological groups, J. Funct. Anal. 225 (2005), 263-300.

[22] - - - Weak amenability of Banach algebras on locally compact groups, ibid. 145 (1997), 175-204.

[23] A. T.-M. Lau and A. Ülger, Topological centers of certain dual algebras, Trans. Amer. Math. Soc. 348 (1996), 1191-1212.

[24] J. Lindenstrauss and L. Tzafriri, Classical Banach Spaces. I, Springer, Berlin, 1977.

[25] T. W. Palmer, Banach Algebras and the General Theory of *algebras, Vol. 1, Cambridge Univ. Press, Cambridge, 1994.

[26] - Arens multiplication and a characterization of $w^{*}$-algebras, Proc. Amer. Math. Soc. 44 (1974), 81-87.

[27] A. Pełczyński, On the isomorphism of the spaces $m$ and $M$, Bull. Acad. Polon. Sci. Sér. Sci. Math. Astronom. Phys. 6 (1958), 695-696.

[28] V. Runde, Dual Banach algebras: Connes-amenability, normal, virtual diagonals, and injectivity of the predual bimodule, Math. Scand. 95 (2004), 124-144.

[29] —, Lectures on Amenability, Springer, Berlin, 2002.

[30] - Amenability for dual Banach algebras, Studia Math. 148 (2001), 47-66.

[31] R. Ryan, Introduction to Tensor Products of Banach Spaces, Springer, London, 2002.

[32] M. Takesaki, Theory of Operator Algebras I, Springer, New York, 1979.

[33] —, Theory of Operator Algebras III, Springer, Berlin, 2003.

[34] A. Ülger, Erratum to: "Arens regularity of the algebra $A \widehat{\otimes} B$ ", Trans. Amer. Math. Soc. 355 (2003), 3839.

[35] -, Arens regularity of the algebra $A \widehat{\otimes} B$, ibid. 305 (1988), 623-639.

[36] - , Continuity of weakly almost periodic functionals on $L^{1}(G)$, Quart. J. Math. Oxford Ser. (2) 37 (1986), 495-497.

[37] N. J. Young, Periodicity of functionals and representations of normed algebras on reflexive spaces, Proc. Edinburgh Math. Soc. (2) 20 (1976/77), 99-120.

St. John's College

Oxford, OX1 3JP, UK

E-mail: matt.daws@cantab.net 